\title{
Grupoides de Lie e o teorema de Noether em teoria de campos no âmbito hamiltoniano
}

\author{
Bruno Tadeu Costa
}

TESE APRESENTADA

$\mathrm{AO}$

INSTITUTO DE MATEMÁTICA E ESTATÍSTICA

DA

UNIVERSIDADE DE SÃO PAULO

PARA

OBTENÇÃO DO TÍTULO

$\mathrm{DE}$

DOUTOR EM CIÊNCIAS

\section{Programa: Matemática Aplicada \\ Orientador: Prof. Dr. Frank Michael Forger}

Durante o desenvolvimento deste trabalho

o autor recebeu auxílio financeiro da CAPES

São Paulo, abril de 2015 



\title{
Grupoides de Lie e o teorema de Noether em teoria de campos no âmbito hamiltoniano
}

\begin{abstract}
Esta versão da tese contém as correções e alterações sugeridas pela Comissão Julgadora durante a defesa da versão original do trabalho, realizada em 24/04/2015. Uma cópia da versão original está disponível no Instituto de Matemática e Estatística da Universidade de São Paulo.
\end{abstract}

Comissão Julgadora:

- Prof. Dr. Frank Michael Forger (Orientador) - IME-USP

- Prof. Dr. Ivan Struchiner - IME-USP

- Prof. Dr. Cristián Ortiz - IME-USP

- Prof. Dr. Henrique Bursztyn - IMPA

- Profa. Dra. Maria Amélia Salazar - IMPA 

Dedico esta tese à minha familia e à minha esposa Naiara 



\section{Agradecimentos}

A toda minha família, especialmente aos meus pais Tadeu e Soraia, meus irmãos Márcio e Beatriz e à minha vó Aurea que sempre estiveram comigo. Não posso deixar de dar um agradecimento especial à minha tia Sônia por sempre ter me incentivado a estudar e pela ajuda incondicional.

À minha esposa Naiara pelo companheirismo, incentivo e por sempre me manter no rumo certo.

Ao meu orientador Michael Forger pela competência, dedicação, disponibilidade e além de tudo pela amizade.

Ao meu amigo Luiz H. P. Pêgas pelas produtivas discussões.

Aos membros da comissão julgadora pelas sugestões, encorajamento e conversas no decorrer do trabalho.

Aos meus amigos da UNESP e da USP: Adriano, Luciano, Danillo, Diego, Danilo, Vitor, Moara, Edson, Bruno, Tatiane, Pricila, Eurípedes, Ricardo, Gustavo e Dylene, Marcelo, André, Anderson, Hector, Oscar e Eliane, Juliano e Graciele, Cleber, e todos aqueles que estiveram ao meu lado durante estes anos de estudo.

À CAPES pelo apoio financeiro, sem o qual não seria possível desenvolver esta pesquisa.

A todos que, direta ou indiretamente, me ajudaram a desenvolver este trabalho. 



\section{Resumo}

Neste trabalho, abordamos o conceito de simetria em teoria de campos, no âmbito hamiltoniano - mais precisamente, sua relação com leis de conservação, conforme estabelecida pelo(s) teorema(s) de Noether. Propomos uma visão alternativa àquela normalmente usada na literatura, baseada na substituição de grupos e álgebras de Lie por grupoides e algebroides de Lie. Tradicionalmente, dado um fibrado $E$ de configuração sobre o espaço-tempo $M$ (cujas seções são os campos do modelo sob investigação), simetrias são implementadas pela ação de um grupo de automorfismos de $E$, ou seja, um subgrupo de $\operatorname{Aut}(E)$, no espaço $\Gamma(E)$ das seções de $E$, exigindo-se que o "funcional ação" $S$ seja invariante sob tal ação: neste caso, quando o pertinente subgrupo for de dimensão infinita, surgem graves dificuldades quando queremos tratar de questões de análise e de geometria com rigor matemático. A vantagem principal desta abordagem alternativa provém do fato de que, embora o grupo $\operatorname{Aut}(E)$ e, tipicamente, os subgrupos relevantes, assim como o espaço $\Gamma(E)$, sejam de dimensão infinita, a sua ação é induzida por uma ação de um grupoide de Lie no fibrado pertinente, a qual envolve apenas variedades de dimensão finita e portanto não há qualquer dúvida em relação a questões tais como qual seria a topologia ou estrutura de variedade subjacente ou em qual sentido essa ação deve ser suave. Formulamos o teorema de Noether neste contexto, baseado em uma nova versão da construção da aplicação momento que a cada "gerador de simetrias" que associa uma $(n-1)$-forma

sobre $\vec{J}{ }^{\circledast} E$ cujo "pull-back" com uma seção de $\vec{J}{ }^{\circledast} E$, que é solução das equações de movimento, produz uma $(n-1)$-forma sobre o espaço-tempo, a famosa corrente de Noether, que é conservada, ou seja, fechada.

Palavras-chave: Teoria clássica de campos, Simetrias, Teorema de Noether, Geometria diferencial, Grupoides de Lie. 



\section{Abstract}

In this thesis, we deal with the concept of symmetry in field theory, in the covariant hamiltonian approach - more precisely, its relation with conservation laws, as established by Noether's theorem(s). We propose an alternative view to that normally used in the literature, based on replacing Lie groups and algebras by Lie groupoids and algebroids. Traditionally, given a configuration bundle $E$ over space-time $M$ (whose sections are the fields of the model under investigation), symmetries are implemented by the action of a group of automorphisms of $E$, i.e., a subgroup of $\operatorname{Aut}(E)$, on the space $\Gamma(E)$ of sections of $E$, requiring the "action functional" $S$ to be invariant under that action: in this case, when the pertinent subgroup has infinite dimension, serious difficulties arise when we want to deal with analytical and geometrical questions with mathematical rigor. The main advantage of this alternative approach comes from the fact that, although the group $\operatorname{Aut}(E)$ and, typically, the relevant subgroups, as well as the space $\Gamma(E)$, are infinite-dimensional, its action is induced by the action of a Lie groupoid in the pertinent bundle, which involves only finite-dimentional manifolds and therefore there is no doubt about questions such as what should be the topology or the underlying manifold structure or in what sense this action should be smooth. We formulate the Noether's theorem in this context, based on a new version of the construction of the momentum map that associates a $(n-1)$-form on $\vec{J}^{\circledast} E$ to each "symmetries generator" whose "pull-back" with

a section of $\vec{J}{ }^{\circledast} E$, that is solution of the equations of motion, produces a $(n-1)$-form on the space-time, the famous Noether current, that is conserved, i.e., closed.

Keywords: Classical field theory, Symmetries, Noether's theorem, Differential geometry, Lie groupoids. 



\section{Sumário}

Introdução $\quad$ ix

1 O Teorema de Noether na Mecânica $\quad 1$

$\begin{array}{lll}2 & \text { Preliminares } & 7\end{array}$

2.1 Jatos e Cojatos . . . . . . . . . . . . . . . . . 7

2.2 Transformação de Legendre . . . . . . . . . . . . . . . . . . . . 18

2.3 Grupoides e Algebroides de Lie . . . . . . . . . . . . . . . . . . . . . . 20

2.3.1 Grupoides de Lie . . . . . . . . . . . . . . . . 20

2.3.2 Algebroides de Lie . . . . . . . . . . . . . . . 33

3 Resultados $\quad 43$

3.1 Ações induzidas . . . . . . . . . . . . . . . . . . . . . . . 45

3.1 .1 Fibrado vertical . . . . . . . . . . . . . . . . . 45

3.1.2 Fibrados dos jatos e descendentes . . . . . . . . . . . . . . . 46

3.1.3 Fibrado tangente (parte 1) . . . . . . . . . . . . 49

3.1.4 Fibrado tangente (parte 2) e descendentes . . . . . . . . . . 50

3.2 Formas Estruturais Invariantes . . . . . . . . . . . . . . . 54

3.3 O Teorema de Noether . . . . . . . . . . . . . . . . . . . . . 59

$\begin{array}{ll}\text { A Grupoides de Pfaff } & 61\end{array}$

$\begin{array}{ll}\text { Bibliografia } & 69\end{array}$ 



\section{Introdução}

A meta principal deste trabalho é dar continuidade ao desenvolvimento de uma visão alternativa do conceito de simetria e sua relação com leis de conservação na teoria geométrica dos campos, conforme estabelecida pelo(s) teorema(s) de Noether, baseada na substituição de grupos e álgebras de Lie por grupoides e algebroides de Lie.

Na formulação das teorias fundamentais da Física, o princípio de invariância sob algum tipo de simetria tem desde sempre ocupado uma posição central (basta pensar em invariância de Galilei na mecânica Newtoniana ou de Lorentz na mecânica relativística) e pode até funcionar como pilar para a construção da teoria, como é o caso do princípio de covariância geral em relatividade geral ou do princípio de invariância de calibre em teorias de calibre. A forma de sua implementação matemática varia de acordo com o contexto físico e assim pode envolver entes matemáticos de diversos tipos, dentre os quais podemos citar, a título de exemplo: grupos finitos (para simetrias discretas) e grupos topológicos (para simetrias contínuas) ou, mais especificamente, grupos e álgebras de Lie. ${ }^{1}$ Nesta visão, também se tornam relevantes grupoides e algebroides de Lie, na medida em que levamos em consideração a possibilidade de existirem restrições ao compor duas transformações de simetria para se chegar a uma terceira.

Nas aplicações à mecânica - em todas as suas versões, relativística ou não, clássica ou quântica - a teoria de grupos e álgebras de Lie e de suas representações ou, mais geralmente, suas ações, têm proporcionado um arcabouço matemático suficientemente amplo para descrever simetrias contínuas. Porém, em teoria dos campos, aparece um ingrediente novo: o princípio de localidade em relação ao espaço-tempo. Por exemplo, observáveis são geralmente associadas a regiões finitas no espaço-tempo e não, indiscriminadamente, ao espaço-tempo inteiro: para qualquer sistema físico, podemos medir a sua energia em alguma região finita do espaço (e não no universo inteiro), e ainda em algum instante, ou talvez a sua média temporal, mas referente a um intervalo de tempo finito (e não à toda eternidade). Então por quê não deveríamos associar o mesmo grau de localização

\footnotetext{
${ }^{1} \mathrm{E}$ ainda nem incluímos nesta lista alguns conceitos um pouco mais exóticos, como supergrupos e superálgebras de Lie ou os "grupos quânticos".
} 
também às transformações de simetria que aplicamos a tal sistema? É essa questão que, há quase um século, levou Hermann Weyl a conceber a ideia de uma transformação de calibre. Poucos anos depois, Brandt introduziu o conceito de grupoide, que formaliza a ideia de associar a uma transformação de simetria um "ponto de partida" (a fonte ou "source") e um "ponto de chegada" (o alvo ou "target") e permitir a composição de tais transformações apenas quando o ponto de chegada da primeira coincide com o ponto de partida da segunda.

Um outro ponto de destaque na história da área foi a descoberta do elo entre simetrias e a existência de quantidades conservadas, o qual hoje conhecemos, genericamente, como o(s) teorema(s) de Noether. Na mecânica clássica, uma formulação matemática precisa no âmbito da geometria diferencial pode ser dada usando o conceito da aplicação momento ("momentum map") [1]. Porém, em teoria dos campos, a situação é menos clara, pois até recentemente faltavam alguns pré-requisitos, tais como uma formulação hamiltoniana covariante (i.e., compatível com os princípios da relatividade - tanto restrita como geral).

$\mathrm{Na}$ teoria geométrica dos campos, os objetos fundamentais - os campos - são descritos por seções de algum fibrado $E$ sobre uma variedade base $n$-dimensional $M$ que representa o espaço-tempo, o que viabiliza a implementação do princípio de localização no espaçotempo. Por exemplo, podemos restringir campos a abertos de $M$ ou, mais radicalmente, considerar suas expansões de Taylor em torno dos pontos de $M$, levando à introdução de conceitos matemáticos tais como feixes e jatos. Assim, no âmbito do formalismo de primeira ordem adotado neste trabalho, aparece naturalmente o fibrado $J E$ dos jatos de primeira ordem de $E$, cujas seções (holônomas) correspondem às derivadas de primeira ordem das seções do fibrado $E$ original. ${ }^{2}$ Observando que $J E$ é um fibrado afim em relação à projeção alvo sobre $E$, passamos ao fibrado $J^{\star} E$ dos cojatos comuns, que é seu dual afim, e em seguida ao fibrado $J^{\circledast} E$ dos cojatos torcidos, que é o produto tensorial deste com o fibrado em linhas $\bigwedge^{n} T^{*} M$ das formas de volume sobre $M$ (ou mais exatamente, seu "pull-back" para $E$ ),

$$
J^{\circledast} E=J^{\star} E \otimes \pi_{E}^{*}\left(\bigwedge^{n} T^{*} M\right),
$$

sendo que ambos são fibrados vetoriais em relação à projeção alvo sobre $E$. De modo semelhante, define-se o fibrado $\vec{J} E$ dos jatos linearizados de primeira ordem de $E$, que é o fibrado vetorial de diferenças associado ao fibrado afim $J E$, e os pertinentes duais: o fibrado $\overrightarrow{J^{*}} E$ dos cojatos linearizados comuns, que é seu dual linear, e em seguida o fibrado

\footnotetext{
${ }^{2}$ Em um formalismo de ordem superior, usa-se o fibrado $J^{r} E$ dos jatos de ordem $r$ de $E$, cujas seções (holônomas) correspondem às derivadas de ordem $\leqslant r$ das seções do fibrado $E$ original, mas por analogia com o que costuma ser feito já no caso da mecânica geométrica clássica, não consideraremos este tipo de situação aqui.
} 
$\vec{J}{ }^{\circledast} E$ dos cojatos linearizados torcidos, que é o produto tensorial deste com $\wedge^{n} T^{*} M$ (ou mais exatamente, seu "pull-back" para $E$ ),

$$
\vec{J}^{\circledast} E=\vec{J}^{*} E \otimes \pi_{E}^{*}\left(\bigwedge^{n} T^{*} M\right),
$$

sendo que, novamente, ambos são fibrados vetoriais em relação à projeção alvo sobre $E$. Ademais, prova-se que $J^{\circledast E}$ é um fibrado afim em linhas sobre $\vec{J}{ }^{\circledast} E$, cuja projeção denotaremos por $\eta$, digamos, e que a hamiltoniana da teoria corresponde a uma seção $\mathcal{H}: \vec{J}{ }^{\circledast} E \longrightarrow J^{\circledast} E$ deste fibrado de tal modo que, se efetuarmos uma transformação de Legendre covariante que consiste de duas aplicações $\mathbb{F} \mathcal{L}: J E \longrightarrow J^{\circledast} E$ e $\overrightarrow{\mathbb{F}} \mathcal{L}: J E \longrightarrow \vec{J}^{\circledast} E$, então no caso de um sistema não-degenerado, $\mathcal{H}$ é unicamente determinada pela condição de que o seguinte diagrama comuta:

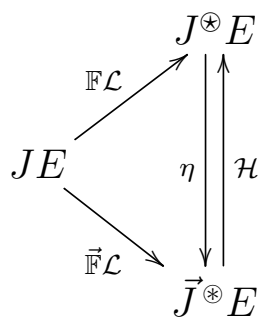

A seguir, usamos os termos "espaço de multifase comum" para $\vec{J}{ }^{\circledast} E$ e "espaço de multifase estendido" para $J^{\circledast} E$ : o segundo vem munido de uma $(n+1)$-forma $\omega$, chamada de forma multissimplética, que (a menos de um sinal que é meramente convencional) é a derivada exterior de uma $n$-forma $\theta$, chamada de forma multicanônica, e dada uma determinada hamiltoniana $\mathcal{H}$, estas induzem o mesmo tipo de forma no primeiro, por "pull-back". Podemos chamar as formas $\theta$ e $\omega$ sobre $J^{\circledast} E$ de "cinemáticas" e as formas $\theta_{\mathcal{H}}=\mathcal{H}^{*} \theta$ e $\omega_{\mathcal{H}}=\mathcal{H}^{*} \omega$ sobre $\vec{J}^{\circledast} E$ de "dinâmicas", pois as últimas dependem da escolha da hamiltoniana e as primeiras não. Para maiores detalhes sobre este formalismo multissimplético, veja $[2,7,10]$.

Passando à questão da descrição de simetrias e de sua relação com leis de conservação, constata-se que a noção de simetria não costuma ser formulada neste mesmo nível "totalmente local". Tradicionalmente, simetrias são dadas pela ação de um grupo de automorfismos de $E$ - ou seja, um subgrupo de $\operatorname{Aut}(E)$ - no espaço $\Gamma(E)$ das seções de $E$, exigindo-se que o "funcional ação" $S$ (o qual determina a dinâmica da teoria) seja invariante sob tal ação. Tal grupo pode ser de dimensão finita, como o grupo de Lorentz no âmbito da relatividade restrita ou grupos de simetrias internas globais, mas também pode ser de dimensão infinita, como acontece no caso de simetrias locais (em particular, simetrias de calibre): neste caso, surgem graves dificuldades quando queremos tratar de questões de análise e de geometria com rigor matemático. A visão alternativa mencionada 
no início consiste em considerar tais ações de grupo como induzidas pela ação de um certo grupoide de Lie sobre $M$ em um (ou vários) dos acima mencionados fibrados, deixando a lagrangiana $\mathcal{L}$ ou hamiltoniana $\mathcal{H}$ invariante. A vantagem principal desta abordagem provém do fato que, embora o grupo $\operatorname{Aut}(E)$ e, tipicamente, os subgrupos pertinentes, assim como o espaço $\Gamma(E)$, sejam de dimensão infinita, a ação do grupoide de Lie pertinente no fibrado pertinente envolve apenas variedades de dimensão finita e portanto não há qualquer dúvida em relação a questões tais como qual seria a topologia ou estrutura de variedade subjacente ou em qual sentido essa ação deve ser suave.

Em um trabalho anterior desenvolvido no grupo de pesquisa do qual o autor faz parte, dando origem a uma tese de doutorado [22] cujos resultados principais foram publicados recentemente [8], foi dado um primeiro passo para concretizar este programa de "redução de simetrias locais a dimensão finita", no âmbito do formalismo lagrangiano e para o caso particular de fibrados de grupos de Lie (que podem ser vistos como grupoides de Lie totalmente intransitivos), o que permite tratar de simetrias locais "internas", ou simetrias de calibre. Faltou estender o procedimento ao âmbito do formalismo hamiltoniano covariante e para o caso de grupoides de Lie em geral, a fim de incluir simetrias locais que agem não-trivialmente no espaço-tempo.

Abordando estas questões, logo encontramos algumas dificuldades conceituais que, ao contrário do que se esperava inicialmente, acabaram obstruindo o progresso durante muito tempo, mas que agora parecem poder ser superadas.

As dificuldades conceituais mencionadas no parágrafo anterior decorrem da questão de como interpretar as expressões "ação induzida" de um grupoide de Lie e "invariância" de uma forma diferencial (ou mais geralmente, de um campo tensorial qualquer) sob a ação de um grupoide de Lie - dificuldades que inexistem no caso de grupos de Lie. De fato, dada uma ação de um grupo de Lie $G$ em uma variedade $X$, obtemos de maneira natural uma ação induzida do mesmo grupo de Lie $G$ no fibrado tangente $T X$ de $X$ e, por conseguinte, em todos os seus descendentes $T_{s}^{r} X,{ }^{3}$ o que permite definir, de maneira óbvia, quando uma forma diferencial sobre $X$ (ou mais geralmente, um campo tensorial qualquer sobre $X$ ) deve se chamar de $G$-invariante. De modo semelhante, dada uma ação de um grupo de Lie $G$ em um fibrado $E$ sobre uma variedade $M$, obtemos de maneira natural uma ação induzida do mesmo grupo de Lie $G$ no fibrado de jatos de primeira ordem de $E, J E$, no fibrado dos jatos linearizados de primeira ordem de $E, \vec{J} E$, e nos seus respectivos duais, comuns ou torcidos.

Nada disso é verdade se, neste tipo de afirmação, substituirmos o termo "grupo de

\footnotetext{
${ }^{3}$ Fibrados vetoriais construídos a partir de um fibrado vetorial dado $V$ mediante um procedimento funtorial aplicado a cada fibra [15, Chapter 3.4], tais como seu dual $V^{*}$ e, mais geralmente, suas potencias tensoriais $V_{s}^{r}$, serão chamadas seus "descendentes".
} 
Lie" pelo termo "grupoide de Lie". Sendo assim, está longe de ser claro o que significaria a afirmação de que, dada uma ação de um grupoide de Lie $G$ em um fibrado $E$ (ambos sobre a mesma variedade base $M$ ), as formas $\theta$ e $\omega$ (ou $\theta_{\mathcal{H}}$ e $\omega_{\mathcal{H}}$ ) que aparecem no formalismo hamiltoniano covariante, seriam invariantes sob tal ação.

A chave para a resposta consiste em perceber que, no caso dos grupoides de Lie, o que se faz necessário para chegar à noção de "ação induzida" é adaptar o próprio grupoide de Lie. Mais concretamente, suponha que seja dada uma ação de um grupoide de Lie $G$ em um fibrado $E$, ambos sobre a mesma variedade base $M$. Então é bem conhecido que o fibrado tangente $T G$ de $G$ é novamente um grupoide de Lie e o fibrado tangente $T E$ de $E$ é novamente um fibrado, porém ambos sobre o fibrado tangente $T M$ de $M$ como variedade base comum, e aí sim temos uma ação induzida, de $T G$ em $T E$, assim como em todos os seus descendentes $T_{s}^{r} E$. No entanto, essa construção é de valor limitado pois altera a variedade base pertinente, de $M$ para $T M$. De modo semelhante, existe uma pequena modificação da construção do fibrado de jatos que associa ao grupoide de Lie $G$ outro grupoide de Lie $J G$, desta vez sobre a mesma variedade base $M$ que o grupoide original $G$ : o correspondente grupoide dos jatos de primeira ordem de $G$. Além disso, a ação original de $G$ em $E$ induz uma ação de $J G$ no fibrado dos jatos $J E$ de $E$, assim como nos seus descendentes. ${ }^{4}$ Mas o que não parece ser de conhecimento geral é que ainda existe uma outra maneira de construir uma ação induzida, desta vez de $J G$ em $T E$, assim como em todos os seus descendentes $T_{s}^{r} E$, considerados como fibrados sobre $M$, e é esta ação induzida que se revela ser a ferramenta essencial para superar as dificuldades conceituais mencionadas acima: a invariância de uma forma diferencial (ou mais geralmente, de um campo tensorial qualquer) sobre $E$ se refere a essa ação de $J G$ e, de modo geral, se dá sob algum subgrupoide de Lie de $J G$ !

Esta tese está organizada da seguinte maneira:

No Capítulo 1, analisamos uma "versão bebê" da teoria a ser desenvolvida, que se aplica no âmbito da mecânica clássica, revendo a formulação do teorema de Noether neste contexto.

No Capítulo 2, apresentamos vários conceitos que são fundamentais para o que será desenvolvido no capítulo seguinte: fibrados de jatos e de cojatos, a transformação de Legendre e elementos da teoria dos grupoides e algebroides de Lie.

No Capítulo 3, encontram-se os resultados principais desta tese. Primeiro, explicamos como uma ação de um grupoide de Lie $G$ sobre um fibrado $E$ induz ações do seu grupoide

\footnotetext{
${ }^{4}$ De forma semelhante como antes, entendemos que os "descendentes" de $J E$ são o fibrado dos jatos linearizados $\vec{J} E$ de $E$, assim como os respectivos duais (comuns e torcidos), $J^{\star} E, \vec{J}^{\star} E, J^{\circledast} E$ e $\vec{J} \circledast E$, e possíveis potencias tensoriais destes.
} 
dos jatos $J G$ sobre todos os fibrados que, em algum sentido, podem ser derivados a partir dele, tais como o fibrado vertical $V E$, o fibrado dos jatos $J E$ e o dos jatos linearizados $\vec{J} E$, o fibrado tangente $T E$ e, finalmente, todos os seus descendentes, como já foi mencionado acima. Em seguida, provamos o primeiro resultado importante, que estabelece invariância das formas "cinemáticas" $\theta$ e $\omega$ sobre $J^{\circledast} E$ sob a ação induzida de $J G$ (mais exatamente, sob certos subgrupoides de Lie de $J(J G)$ ): trata-se do análogo do bem-conhecido teorema da geometria simplética afirmando que, dada qualquer variedade $Q$ munida de qualquer ação de qualquer grupo de Lie $G$, as formas $\theta$ e $\omega$ sobre o fibrado cotangente $T^{*} Q$ de $Q$ são invariantes sob a ação induzida de $G$. Quanto às formas "dinâmicas" $\theta_{\mathcal{H}}$ e $\omega_{\mathcal{H}}$ sobre $\vec{J}{ }^{\circledast} E$, estabelecemos resultados análogos de invariância em relação à ação de um subgrupoide de Lie $\tilde{G}$ de $J G$ que deixa a hamiltoniana $\mathcal{H}$ invariante (mais exatamente, em relação a certos subgrupoides de Lie de $J \tilde{G})$. Finalmente, formulamos na última seção o teorema de Noether neste contexto, baseado em uma nova versão da construção da aplicação momento que a cada "gerador de simetria" associa uma $(n-1)$-forma sobre $\vec{J}{ }^{\circledast} E$ cujo "pull-back" com uma seção de $\vec{J}{ }^{\circledast} E$, que é solução das equações de movimento, produz uma $(n-1)$-forma sobre o espaço-tempo, a famosa corrente de Noether, que é conservada, ou seja, fechada.

No Apêndice A, discutimos brevemente o conceito de um grupoide de Pfaff, que é um grupoide de Lie munido de uma certa estrutura adicional no seu grupoide tangente. Em particular, mostramos que existe uma estrutura canônica não-trivial de grupoide de Pfaff em qualquer grupoide de Lie da forma $J G$ onde $G$ é outro grupoide de Lie, e relacionamos essa estrutura com a do grupoide dos jatos de segunda ordem de $G$. 


\section{Capítulo 1}

\section{O Teorema de Noether na Mecânica}

Na abordagem geométrica à mecânica clássica (não-relativística), a relação entre simetrias e leis de conservação, da qual trata o teorema de Noether, é formulada da seguinte maneira [1,21]:

Considere primeiro uma variedade conexa $P$ que representa o espaço de fase do sistema mecânico sob consideração, munida de uma forma simplética $\omega$. Os pontos de $P$ representam os estados puros e as funções suaves sobre $P$ as observáveis do sistema, que formam uma álgebra de Poisson $\mathfrak{F}(P){ }^{1}$ Então na álgebra de Lie $\mathfrak{X}(P)$ dos campos vetoriais sobre $P$, temos as subálgebras $\mathfrak{X}_{L H}(P)$ dos campos localmente hamiltonianos e $\mathfrak{X}_{H}(P)$ dos campos vetoriais hamiltonianos sobre $P$, definidas por

$$
X \in \mathfrak{X}_{L H}(P) \Longleftrightarrow i_{X} \omega \text { é uma 1-forma fechada }
$$

e

$$
X \in \mathfrak{X}_{H}(P) \Longleftrightarrow i_{X} \omega \text { é uma 1-forma exata }
$$

onde se nota que

$$
X \in \mathfrak{X}_{L H}(P) \Longleftrightarrow L_{X} \omega=0
$$

e que $\mathfrak{X}_{H}(P)$ é um ideal em $\mathfrak{X}_{L H}(P)$. Ademais, a cada função $f \in \mathfrak{F}(P)$, associamos o campo vetorial hamiltoniano $X_{f} \in \mathfrak{X}_{H}(P)$ obtido como o dual simplético da sua derivada exterior, explicitamente dado por

$$
i_{X_{f}} \omega=d f .
$$

\footnotetext{
${ }^{1}$ Mediante algumas modificações óbvias, as afirmações deste parágrafo podem ser estendidas ao caso em que $P$ é uma variedade de Poisson, mas para os fins desta tese, que visa a passagem da mecânica para a teoria de campos, será suficiente se restringir ao arcabouço da geometria simplética. Por outro lado, uma generalização importante para viabilizar essa passagem consiste em estender o formalismo de tal forma a incluir sistemas não-autônomos, onde as observáveis do sistema, inclusive a hamiltoniana, podem depender explicitamente do tempo: retornaremos a este assunto mais adiante.
} 
Assim, considerando $\mathfrak{F}(P)$ como álgebra de Lie em relação ao colchete de Poisson definido por

$$
\{f, g\}=\omega\left(X_{f}, X_{g}\right)
$$

obtemos a seguinte sequência exata de álgebras de Lie:

$$
\{0\} \longrightarrow \mathbb{R} \longrightarrow \mathfrak{F}(P) \longrightarrow \mathfrak{X}_{H}(P) \longrightarrow\{0\} \text {. }
$$

Então a dinâmica de um determinado modelo é fixada pela escolha de uma função específica $H \in \mathfrak{F}(P)$, chamada de hamiltoniana, tal que a evolução do sistema no tempo é dada pelo fluxo do campo $X_{H}$.

Neste âmbito, simetrias de um sistema são descritas por uma ação

$$
\begin{aligned}
G \times P & \longrightarrow P \\
(g, p) & \longmapsto g \cdot p
\end{aligned}
$$

de algum grupo de Lie $G$ no espaço de fase $P$ que preserva a forma simplética e a hamiltoniana. Ou seja, para todo $g \in G$, vale

$$
L_{g}^{*} \omega=\omega
$$

e

$$
L_{g}^{*} H=H,
$$

onde $L_{g}^{*}$ denota o "pull-back" pela ação à esquerda $L_{g}: P \rightarrow P$ por $g$, definida por $L_{g}(p)=g \cdot p$. Tal ação induz uma ação infinitesimal da álgebra de Lie $\mathfrak{g}$ de $G$, i.e., um homomorfismo de álgebras de Lie

$$
\begin{array}{ccc}
\mathfrak{g} & \longrightarrow & \mathfrak{X}_{L H}(P) \\
X & \longmapsto & X_{P}
\end{array}
$$

onde $X_{P}$ denota o campo fundamental localmente hamiltoniano associado a $X$ :

$$
X_{P}(p)=\left.\frac{d}{d t} \exp (t X) \cdot p\right|_{t=0}
$$

Portanto uma aplicação momento para essa simetria é um homomorfismo de álgebras de Lie

$$
\begin{aligned}
\mathcal{J}: \mathfrak{g} & \longrightarrow \mathfrak{F}(P) \\
X & \longmapsto \mathcal{J}(X)
\end{aligned}
$$

tal que, para todo $X \in \mathfrak{g}$, vale

$$
i_{X_{P}} \omega=d \mathcal{J}(X)
$$


Ou seja, o seguinte diagrama comuta:

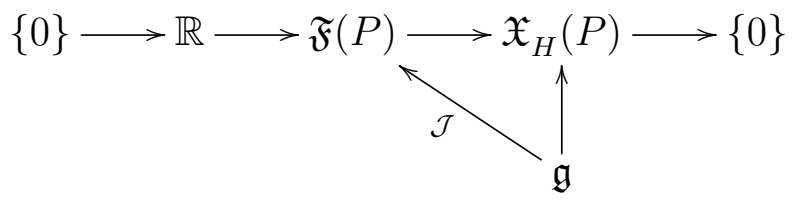

Para encontrar uma construção análoga em teoria de campos, precisamos primeiro discutir o caso especial em que $P$ é um fibrado cotangente, onde é sempre possível construir uma aplicação momento explícita.

Considere então uma variedade $Q$ que representa o espaço de configuração para o sistema mecânico sob consideração, de modo que o correspondente espaço de fase é o fibrado cotangente $T^{*} Q$ de $Q$, que vem munido de uma forma canônica $\theta$ da qual a forma simplética $\omega$ é, a menos de um sinal, a derivada exterior $(\omega=-d \theta)$; explicitamente, em coordenadas de Darboux,

$$
\theta=p_{i} d q^{i}
$$

$\mathrm{e}$

$$
\omega=d q^{i} \wedge d p_{i}
$$

Neste caso, simetrias do sistema são descritas por uma ação

$$
\begin{aligned}
G \times Q & \longrightarrow Q \\
(g, q) & \longmapsto g \cdot q
\end{aligned}
$$

de algum grupo de Lie $G$ no seu espaço de configuração $Q$, sendo que a ação anterior de $G$ no espaço de fase é obtida passando primeiro à ação induzida de $G$ no fibrado tangente,

$$
\begin{array}{ccc}
G \times T Q & \longrightarrow & T Q \\
(g,(q, \dot{q})) & \longmapsto & (g \cdot q, g \cdot \dot{q})
\end{array}
$$

por diferenciação, e em seguida à ação induzida de $G$ no fibrado cotangente,

$$
\begin{aligned}
& G \times T^{*} Q \quad \longrightarrow \quad T^{*} Q \\
& (g,(q, p)) \longmapsto(g \cdot q, g \cdot p)
\end{aligned}
$$

por dualização. ${ }^{2}$ Observa-se então que esta ação induzida é automaticamente simplética e até exatamente simplética, i.e., preserva não apenas a forma simplética $\omega$ mas também a forma canônica $\theta$, ou seja, para todo $g \in G$, vale, além da equação (1.8), a condição

$$
L_{g}^{*} \theta=\theta
$$

\footnotetext{
${ }^{2}$ Aqui, efetuamos uma ligeira mudança de notação, escrevendo os pontos do espaço de fase na forma de pares $(q, p)$, onde $q$ é um ponto do espaço de configuração e $p$ é um covetor neste ponto.
} 
Essas ações também induzem ações infinitesimais correspondentes da álgebra de Lie $\mathfrak{g}$ de $G$, i.e., homomorfismos de álgebras de Lie

$$
\begin{aligned}
& \mathfrak{g} \longrightarrow \mathfrak{X}(Q) \\
& X \longmapsto X_{Q}
\end{aligned}
$$

$\mathrm{e}$

$$
\begin{aligned}
& \mathfrak{g} \longrightarrow \mathfrak{X}(T Q) \quad \mathfrak{g} \longrightarrow \mathfrak{X}\left(T^{*} Q\right) \\
& X \longmapsto X_{T Q} \quad, \quad X \longmapsto X_{T^{*} Q}
\end{aligned}
$$

por campos fundamentais, de modo que $X_{T Q}$ e $X_{T^{*} Q}$ podem ser obtidos a partir de $X_{Q}$ pelo processo de levantamento canônico, que é a versão infinitesimal do levantamento canônico de difeomorfismos (locais) de $Q$ para automorfismos (locais) de $T Q$ e de $T^{*} Q$. Notamos, de passagem, que este processo de levantamento canônico proporciona homomorfismos de álgebras de Lie

$$
\begin{array}{cccccc}
\mathfrak{X}(Q) & \longrightarrow & \mathfrak{X}(T Q) & \mathfrak{X}(Q) & \longrightarrow & \mathfrak{X}\left(T^{*} Q\right) \\
Z & \longmapsto & Z_{T Q} & Z & \longmapsto & Z_{T^{*} Q}
\end{array}
$$

que, em coordenadas locais adaptadas, assumem a seguinte forma: se

$$
Z=Z^{i} \frac{\partial}{\partial q^{i}}
$$

então vale

$$
Z_{T Q}=Z^{i} \frac{\partial}{\partial q^{i}}+\dot{q}^{j} \frac{\partial Z^{i}}{\partial q^{j}} \frac{\partial}{\partial \dot{q}^{i}}, \quad Z_{T^{*} Q}=Z^{i} \frac{\partial}{\partial q^{i}}-p_{j} \frac{\partial Z^{j}}{\partial q^{i}} \frac{\partial}{\partial p_{i}} .
$$

Ocorre, então, que a forma canônica $\theta$ proporciona uma aplicação momento canônica "universal"

$$
\begin{aligned}
\mathcal{J}_{\text {univ }}: \mathfrak{X}(Q) & \longrightarrow \mathfrak{F}\left(T^{*} Q\right) \\
Z & \longmapsto \mathcal{J}_{\text {univ }}(Z)
\end{aligned}
$$

definida por

$$
\mathcal{J}_{\text {univ }}(Z)=i_{Z_{T^{*} Q}} \theta
$$

ou, explicitamente, em coordenadas de Darboux,

$$
\mathcal{J}_{\text {univ }}(Z)=p_{i} Z^{i}
$$

tal que, para qualquer grupo de Lie $G$ e qualquer ação de $G$ sobre o espaço de configuração $Q$, a composição de $\mathcal{J}_{\text {univ }}$ com a correspondente ação infinitesimal de $\mathfrak{g}$ proporciona uma aplicação momento $\mathcal{J}$ para essa simetria. 
Um outro aspecto de importância fundamental em teoria de campos, mas pouco explorado na mecânica, concerne à distinção entre "simetrias globais" e "simetrias locais". Como foi mostrado em [5, Sect. 5], simetrias locais podem ser incorporadas se estendermos a ação (1.18) no fibrado tangente a uma ação do grupo tangente $T G$ de $G$, que pode ser identificado com o produto semidireto $G \ltimes \mathfrak{g}$ de $G$ com a sua própria álgebra de Lie, considerada como grupo abeliano, mediante a ação do primeiro fator sobre o segundo pela representação adjunta, com produto definido por

$$
\left(g_{1}, X_{1}\right)\left(g_{2}, X_{2}\right)=\left(g_{1} g_{2}, X_{1}+\operatorname{Ad}\left(g_{1}\right) X_{2}\right)
$$

definida da seguinte forma:

$$
\begin{array}{llc}
(G \ltimes \mathfrak{g}) \times T Q & \longrightarrow & T Q \\
((g, X),(q, \dot{q})) & \longmapsto & (g, X) \cdot(q, \dot{q})
\end{array}
$$

com

$$
(g, X) \cdot(q, \dot{q})=\left(g \cdot q, g \cdot \dot{q}+X_{Q}(g \cdot q)\right)
$$

Contudo, como essa ação é apenas afim e não linear, ela não pode ser transferida a uma ação no fibrado cotangente, sendo que a implementação matemática correta de tal abordagem no lado hamiltoniano é muito menos óbvia do que no lado lagrangiano, mesmo no contexto da mecânica. Claramente, a dificuldade aqui encontrada está relacionada com o fato de que sistemas com simetrias locais são necessariamente degenerados e portanto não existe nenhuma equivalência óbvia entre as suas formulações lagrangianas e hamiltonianas. Trata-se de um dos itens a serem abordados no desenvolvimento do trabalho.

Finalizamos o capítulo com o enunciado e a prova do teorema de Noether na mecânica clássica, que assegura a existência de uma correspondência biunívoca entre simetrias e integrais de movimento:

Teorema 1.1 Seja $(P, \omega)$ uma variedade simplética munida de uma ação de um grupo de Lie $G$ que preserva a forma simplética $\omega$. Se $H: P \longrightarrow \mathbb{R}$ é uma função $G$-invariante, então a aplicação momento $\mathcal{J}$ é constante ao longo das trajetórias do campo vetorial hamiltoniano de $H$. 
Demonstração: Seja $X_{H}$ o campo hamiltoniano de $H$. Dado $X \in \mathfrak{g}$, temos:

$$
L_{X_{H}} \mathcal{J}(X)=i_{X_{H}} d \mathcal{J}(X)=i_{X_{H}} i_{X_{P}} \omega=-i_{X_{P}} i_{X_{H}} \omega=-i_{X_{P}} d H=-L_{X_{P}} H=0,
$$

pois $H$ é $G$-invariante.

Nas hipóteses do teorema, a tripla $(P, \omega, H)$ constitui o que é conhecido como um "G-espaço hamiltoniano", e o teorema assegura que nesta situação, todas as funções da forma $\mathcal{J}(X)$, para $X \in \mathfrak{g}$, são integrais de movimento deste sistema hamiltoniano.

Observação: Como podemos observar, a prova do teorema de Noether é um tanto quanto simples na mecânica clássica. No Capítulo 3 deste trabalho, apresentaremos a prova do teorema de Noether na teoria de campos, onde temos a ação de um grupoide de Lie para descrever as simetrias. Como é de se esperar, essa prova não é mais tão simples assim, principalmente pelo fato da imagem da aplicação momento poder ser uma forma com grau maior ou igual a um. Além disso, devemos enfatizar que o enunciado do teorema de Noether na teoria de campos será dado considerando a ação induzida do jato do grupoide de Lie original sobre uma variedade multissimplética específica, a saber, o dual afim torcido. Esses elementos serão definidos no próximo capítulo. Porém, um resultado em [9, Sect. 9] garante que toda variedade multissimplética é difeomorfa ao dual afim torcido, caso sejam satisfeitas algumas condições. Felizmente, todos os exemplos conhecidos satisfazem tais condições. 


\section{Capítulo 2}

\section{Preliminares}

Neste capítulo apresentamos conceitos preliminares essenciais para o desenvolvimento deste trabalho. Discutimos inicialmente o conceito do fibrado de jatos de um fibrado geral, que proporciona o ambiente geométrico correto para acomodar o conjunto das derivadas (parciais) de um campo. Em seguida, introduzimos a transformação de Legendre, ferramenta essencial para a transição do formalismo lagrangiano ao formalismo hamiltoniano, tanto na mecânica como na teoria de campos. Finalizamos com um resumo de aspectos relevantes da teoria de grupoides e algebroides de Lie, dos quais precisaremos quando tratarmos de simetrias no Capítulo 3.

\section{$2.1 \quad$ Jatos e Cojatos}

Nesta seção recordamos a definição de jatos e do fibrado de jatos de um fibrado - conceitos essenciais para o desenvolvimento desta tese. Dadas variedades $M$ e $N$, um ponto qualquer $x$ de $M$ e aplicações

$$
f: M \longrightarrow N \quad \text { e } \quad g: M \longrightarrow N
$$

tais que $f(x)=y=g(x)$, definimos a seguinte relação de equivalência:

$$
\begin{aligned}
& f \text { e } g \text { coincidem até a primeira ordem de suas expansões } \\
& f \sim_{x} g \Longleftrightarrow \text { de Taylor, em quaisquer sistemas de coordenadas locais } \\
& \text { de } M \text { em torno de } x \text { e de } N \text { em torno de } y
\end{aligned}
$$

Devido à regra da cadeia, basta verificar tal relação em um único sistema de coordenadas locais de $M$ em torno de $x$ e um único sistema de coordenadas locais de $N$ em torno de $y$ para garantir que valha em todos. O espaço quociente dado por essa relação 
de equivalência é chamado de espaço dos jatos (de primeira ordem) em $x$. Para uma aplicação $f$ como acima, denotaremos o jato (de primeira ordem) em $x$ por ela induzido por $j_{x} f$ : é apenas uma outra notação para a aplicação tangente $T_{x} f: T_{x} M \longrightarrow T_{y} N$ a $f$ em $x$.

Em particular, podemos falar de jatos não de aplicações entre variedades e sim de seções de fibrados. Dado um fibrado $E$ sobre uma variedade base $M$ com projeção $\pi_{E}: E \longrightarrow M$, para cada ponto $e$ do espaço total $E$, com ponto base $x=\pi_{E}(e)$, define-se o espaço dos jatos (de primeira ordem) em $e$ como o conjunto dos subespaços horizontais de $T_{e} E$, i.e.,

$$
J_{e} E=\left\{H_{e} E \text { subespaço de } T_{e} E \mid T_{e} E=V_{e} E \oplus H_{e} E\right\}
$$

onde

$$
V_{e} E=\operatorname{ker} T_{e} \pi_{E}
$$

é o espaço vertical de $E$ em $e$. Pensando em termos de seções, podemos reformular esta definição de modo a permitir uma abordagem mais prática a este conjunto, notando que uma seção de $E$ é uma aplicação $\varphi: M \longrightarrow E$ que satisfaz $\pi_{E} \circ \varphi=\mathrm{id}_{M}$; portanto, diferenciando esta condição, notamos que seu jato (de primeira ordem) em $x$ é uma aplicação linear $j_{x} \varphi: T_{x} M \longrightarrow T_{\varphi(x)} E$ tal que $T_{\varphi(x)} \pi_{E} \circ j_{x} \varphi=\mathrm{id}_{T_{x} M}$. Assim,

$$
J_{e} E=\left\{u_{e} \in L\left(T_{x} M, T_{e} E\right) \mid T_{e} \pi_{E} \circ u_{e}=\operatorname{id}_{T_{x} M}\right\} .
$$

A equivalência entre as duas definições se estabelece notando que $u_{e}$ determina $H_{e} E$ $\left(H_{e} E=\operatorname{im} u_{e}\right)$ e $H_{e} E$ determina $u_{e}\left(u_{e}=\left(\left.T_{e} \pi_{E}\right|_{H_{e} E}\right)^{-1}\right)$. Uma vantagem desta segunda versão é que ela evidencia que $J_{e} E$ é um subespaço afim do espaço vetorial $L\left(T_{x} M, T_{e} E\right)$, sendo que o correspondente espaço vetorial de diferenças, que chamaremos de espaço dos jatos linearizados (de primeira ordem) em e, é dado por

$$
\overrightarrow{J_{e}} E=\left\{\vec{u}_{e} \in L\left(T_{x} M, T_{e} E\right) \mid T_{e} \pi_{E} \circ \vec{u}_{e}=0\right\} .
$$

Assim, temos

$$
\overrightarrow{J_{e}} E=L\left(T_{x} M, V_{e} E\right) \cong T_{x}^{*} M \otimes V_{e} E
$$

Variando o ponto e, obtemos o fibrado JE dos jatos (de primeira ordem) e o fibrado $\vec{J} E$ dos jatos linearizados (de primeira ordem) de $E$, com

$$
\vec{J} E=L\left(\pi_{E}^{*}(T M), V E\right) \cong \pi_{E}^{*}\left(T^{*} M\right) \otimes V E,
$$

onde $V E$ é o fibrado vertical de $E$. Na verdade, ambos são fibrados em dois sentidos diferentes, pois admitem uma projeção sobre o espaço total do fibrado original que 
denotaremos, respectivamente, por $\pi_{J E}$ e por $\pi_{\overrightarrow{J E}}$, e outra projeção sobre o espaço base do fibrado original, obtida da anterior por composição com a projeção original. ${ }^{1}$ Representamos esta situação pelos seguintes diagramas:
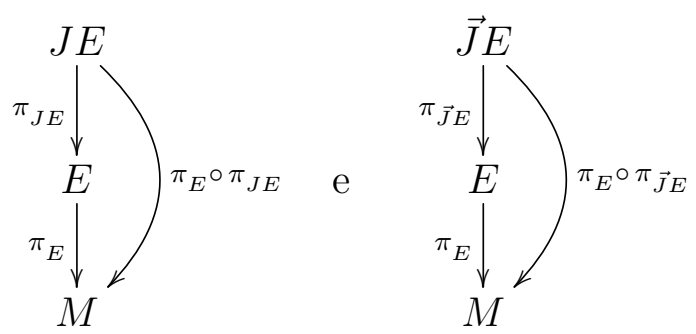

Vale enfatizar que em relação às projeções sobre $E, J E$ é um fibrado afim e $\vec{J} E$ é um fibrado vetorial, enquanto que em relação às projeções sobre $M$, ambos são apenas fibrados gerais quando $E$ é um fibrado geral; no entanto, podem adquirir estrutura extra se $E$ vier munido de estrutura extra. Por exemplo, pode-se mostrar que, se $E$ for um fibrado vetorial sobre $M$, então $J E$ e $\overrightarrow{J E}$ também o são, e como tais podem ser identificados.

Para que essa construção possa ser assimilada mais facilmente, explicamos como ela se expressa em termos de coordenadas e trivializações locais. Considere coordenadas locais $x^{\mu}$ para a variedade $M$ e $q^{i}$ para a variedade $Q$ que são, respectivamente, a base e a fibra típica do fibrado. Em combinação com uma trivialização local de $E$, elas induzem coordenadas locais

- $\left(x^{\mu}, q^{i}\right)$ para $E$,

- $\left(x^{\mu}, q^{i}, q_{\mu}^{i}\right)$ para $J E$,

- $\left(x^{\mu}, q^{i}, \vec{q}_{\mu}^{i}\right)$ para $\overrightarrow{J E}$,

que chamaremos de coordenadas locais adaptadas por serem compatíveis com as respectivas projeções: a projeção $\pi_{E}$, no primeiro caso, e as projeções $\pi_{E} \circ \pi_{J E}$ e $\pi_{E} \circ \pi_{\overrightarrow{J E}}$, nos últimos dois casos, correspondem à projeção sobre a primeira componente (dos pontos com coordenadas $\left(x^{\mu}, q^{i}\right)$ ou $\left(x^{\mu}, q^{i}, q_{\mu}^{i}\right)$ ou $\left(x^{\mu}, q^{i}, \vec{q}_{\mu}^{i}\right)$ para o ponto com coordenadas $\left.x^{\mu}\right)$, enquanto que as projeções $\pi_{J E}$ e $\pi_{\vec{J} E}$, nos últimos dois casos, correspondem à projeção sobre as primeiras duas componentes (dos pontos com coordenadas $\left(x^{\mu}, q^{i}, q_{\mu}^{i}\right)$ ou $\left(x^{\mu}, q^{i}, \vec{q}_{\mu}^{i}\right)$ para o ponto com coordenadas $\left(x^{\mu}, q^{i}\right)$ ), explicitando a estrutura de "fibrado duplo" caracterizado pelos diagramas (2.8). Para uma melhor compreensão, mostra-se útil exibir como

\footnotetext{
${ }^{1} \mathrm{Na}$ literatura sobre jatos, $\pi_{J E}$ é frequentemente chamada de projeção alvo e denotada por $\tau$ ("target projection"), enquanto que $\pi \circ \pi_{J E}$ é frequentemente chamada de projeção fonte ("source projection") e denotada por $\sigma$, mas nesta tese, evitaremos usar essa terminologia, na medida do possível, pois na teoria de grupoides de Lie, os termos "projeção alvo" e "projeção fonte" também são usados, e com significado diferente.
} 
essas coordenadas induzidas se transformam sob uma mudança de coordenadas $x^{\mu} \rightarrow \bar{x}^{\nu}$ na base $M$ e $q^{i} \rightarrow \bar{q}^{j}$ na fibra típica $Q$, acompanhada de uma mudança de trivialização, as quais, conjuntamente, induzem mudanças de coordenadas

$$
\begin{aligned}
& \text { - }\left(x^{\mu}, q^{i}\right) \rightarrow\left(\bar{x}^{\nu}, \bar{q}^{j}\right) \text { para } E, \\
& \text { - }\left(x^{\mu}, q^{i}, q_{\mu}^{i}\right) \rightarrow\left(\bar{x}^{\nu}, \bar{q}^{j}, \bar{q}_{\nu}^{j}\right) \text { para } J E, \\
& \text { - }\left(x^{\mu}, q^{i}, \vec{q}_{\mu}^{i}\right) \rightarrow\left(\bar{x}^{\nu}, \bar{q}^{j}, \vec{q}_{\nu}^{j}\right) \text { para } \overrightarrow{J E},
\end{aligned}
$$

onde a primeira deve satisfazer a condição de que

$$
\bar{x}^{\nu}=\bar{x}^{\nu}\left(x^{\mu}\right) \quad, \quad \bar{q}^{j}=\bar{q}^{j}\left(x^{\mu}, q^{i}\right)
$$

para garantir que a transformação preserva a projeção $\pi_{E}$, enquanto que as últimas duas são dadas por

$$
\bar{q}_{\nu}^{j}=\frac{\partial \bar{q}^{j}}{\partial x^{\mu}} \frac{\partial x^{\mu}}{\partial \bar{x}^{\nu}}+\frac{\partial \bar{q}^{j}}{\partial q^{i}} q_{\mu}^{i} \frac{\partial x^{\mu}}{\partial \bar{x}^{\nu}}
$$

e

$$
\overrightarrow{\bar{q}}_{\nu}^{j}=\frac{\partial \bar{q}^{j}}{\partial q^{i}} \vec{q}_{\mu}^{i} \frac{\partial x^{\mu}}{\partial \bar{x}^{\nu}}
$$

o que deixa explícito que a transformação induzida preserva a estrutura afim nas fibras de $J E$ sobre $E$ e a estrutura linear nas fibras de $\vec{J} E$ sobre $E$.

Um papel essencial em teoria dos fibrados é desempenhado pela noção de seção. No caso do fibrado dos jatos $J E$ de um fibrado $E$ sobre uma variedade base $M$, temos duas projeções e portanto duas noções diferentes de seção.

Quanto às primeiras, notamos que toda seção $\varphi$ de $E$ sobre $M$ induz uma seção de $J E$ sobre $M$ que denotaremos por $j \varphi$ ou, ainda, por $(\varphi, \partial \varphi)$. A primeira notação é padrão na literatura matemática, expressando o fato que $j \varphi$ associa a cada ponto $x$ de $M$ seu jato (de primeira ordem) $j_{x} \varphi \in J_{\varphi(x)} E \subset L\left(T_{x} M, T_{\varphi(x)} E\right)$ em $x$ : em princípio, trata-se apenas de uma reinterpretação da aplicação tangente $T \varphi: T M \longrightarrow T E$ à aplicação $\varphi: M \longrightarrow E$, mas que enfatiza a natureza de seção de ambas. A segunda notação aparece, explicita ou implicitamente, em textos de física, enfatizando o aspecto de que, em coordenadas locais adaptadas $\left(x^{\mu}, q^{i}\right)$, onde a seção $\varphi$ é representada por um conjunto de funções $\varphi^{i}$ das variáveis $x^{\mu}$, a seção $(\varphi, \partial \varphi)$ é representada pelo conjunto das funções $\left(\varphi^{i}, \partial_{\mu} \varphi^{i}\right)$ destas mesmas variáveis $x^{\mu}$. Notamos ainda que, na literatura matemática, a seção $j \varphi$ é frequentemente chamada de prolongamento (de primeira ordem) da seção $\varphi$, mas nós preferimos pensar nela como sendo simplesmente a derivada da seção $\varphi$. Finalmente, cabe mencionar que nem toda seção de $J E$ sobre $M$ pode ser escrita como derivada de uma seção de $E$ sobre $M$ : as que podem são chamadas de seções holônomas. 
Quanto às segundas, observamos que as seções de $J E$ sobre $E$ possuem uma interpretação completamente diferente, pois elas correspondem a conexões no fibrado $E$. De fato, uma seção $\Gamma: E \longrightarrow J E$ define, em cada ponto $e \in E$ com ponto base $x \in M$, uma aplicação linear $\Gamma(e) \in L\left(T_{x} M, T_{e} E\right)$ cuja imagem é o subespaço horizontal $H_{e} E \subset T_{e} E$ da conexão, $H_{e} E=\operatorname{im} \Gamma(e)$, e reciprocamente, dado o subespaço horizontal $H_{e} E \subset T_{e} E$ da conexão, define-se $\Gamma(e) \in L\left(T_{x} M, H_{e} E\right)$ por $\Gamma(e)=\left(\left.T_{e} \pi_{E}\right|_{H_{e} E}\right)^{-1}$. Também é possível, neste âmbito, tratar de classes especiais de conexões, que existem na medida em que o fibrado $E$ vem munido de alguma estrutura adicional: os exemplos mais importantes são conexões lineares em fibrados vetoriais e conexões principais em fibrados principais.

Tendo em vista que o fibrado dos jatos de primeira ordem, assim como o fibrado dos jatos linearizados de primeira ordem, aparece na teoria de campos como um análogo do fibrado tangente do espaço de configuração na mecânica clássica, podemos nos perguntar qual seria o análogo do fibrado cotangente. Obviamente, isto envolve algum processo de dualização que, como veremos, acaba levando a duas (ou no final, a quatro) versões de dual ligeiramente diferentes. Primeiro, introduzimos o dual afim $J^{\star} E$ de $J E$ e o dual linear $\overrightarrow{J^{*}} E$ de $\vec{J} E$, cujas fibras em qualquer ponto $e \in E$ são os espaços vetoriais

$$
J_{e}^{\star} E=\left\{z_{e}: J_{e} E \longrightarrow \mathbb{R} \mid z_{e} \text { é afim }\right\}
$$

e

$$
\overrightarrow{J_{e}^{*}} E=\left\{\vec{z}_{e}: \overrightarrow{J_{e}} E \longrightarrow \mathbb{R} \mid \vec{z}_{e} \text { é linear }\right\}
$$

respectivamente. Porém, o espaço correto para trabalharmos na teoria de campos é o que se chama o dual torcido, obtido quando substituirmos o corpo dos números reais pelo espaço unidimensional das formas de volume sobre o espaço tangente à base $M$ no ponto correspondente. Explicitamente, introduzimos o dual afim torcido $J^{\circledast} E$ de $J E$ e o dual linear torcido $\vec{J}{ }^{\circledast} E$ de $\overrightarrow{J E}$, cujas fibras em qualquer ponto $e \in E$ com ponto base $x \in M$ são os espaços vetoriais

$$
J_{e}^{\circledast} E=\left\{z_{e}: J_{e} E \longrightarrow \bigwedge^{n} T_{x}^{*} M \mid z_{e} \text { é afim }\right\}
$$

e

$$
\vec{J}_{e}^{\circledast} E=\left\{\vec{z}_{e}: \overrightarrow{J_{e}} E \longrightarrow \bigwedge^{n} T_{x}^{*} M \mid \vec{z}_{e} \text { é linear }\right\}
$$

respectivamente. Em outras palavras,

$$
J^{\circledast} E=J^{\star} E \otimes \pi_{E}^{*}\left(\bigwedge^{n} T^{*} M\right)
$$

e

$$
\vec{J}^{\circledast} E=\vec{J}^{*} E \otimes \pi_{E}^{*}\left(\bigwedge^{n} T^{*} M\right)
$$


A seguir, utilizaremos a seguinte terminologia para distinguir entre todas essas versões: $J^{\star} E$ é o fibrado dos cojatos comuns, $\overrightarrow{J^{*}} E$ é o fibrado dos cojatos linearizados comuns, $J^{\circledast} E$ é o fibrado dos cojatos torcidos e $\vec{J}^{\circledast} E$ é o fibrado dos cojatos linearizados torcidos. Como nos casos do fibrado dos jatos e do fibrado dos jatos linearizados, todos esses duais admitem duas projeções: uma projeção sobre o espaço total do fibrado original que denotaremos, respectivamente, por $\pi_{J^{\star} E}$, por $\pi_{\vec{J}^{*} E}$, por $\pi_{J^{\circledast E}}$ e por $\pi_{\vec{J}^{\circledast E}}$, e outra projeção sobre o espaço base do fibrado original, obtida da anterior por composição com a projeção original.

Outra estrutura que é importante para o estabelecimento do formalismo hamiltoniano covariante em teoria de campos é que os duais afins $J^{\star} E$ e $J^{\circledast} E$ são fibrados em linha sobre os duais lineares $\vec{J}^{*} E$ e $\vec{J}^{\circledast} E$, respectivamente. Para ver isso, basta notar que toda aplicação afim entre espaços afins induz, de maneira canônica, uma aplicação linear entre os respectivos espaços vetoriais de diferenças e que as aplicações afins cuja correspondente aplicação linear se anula são exatamente as aplicações constantes. Assim, definindo, para qualquer ponto $e \in E$ com ponto base $x \in M$,

$$
J_{e}^{c} E=\left\{z_{e}: J_{e} E \longrightarrow \mathbb{R} \mid z_{e} \text { é constante }\right\}
$$

$\mathrm{e}$

$$
J_{e}^{\odot} E=\left\{z_{e}: J_{e} E \longrightarrow \bigwedge^{n} T_{x}^{*} M \mid z_{e} \text { é constante }\right\}
$$

obtemos subfibrados em linha $J^{c} E$ de $J^{\star} E$ e $J^{\circledR} E$ de $J^{\circledast} E$ tais que

$$
J^{\star} E / J^{c} E \cong \vec{J}^{*} E
$$

e

$$
J^{\circledast} E / J^{\circledR} E \cong \vec{J}^{\circledast} E
$$

A partir de coordenadas locais adaptadas $\left(x^{\mu}, q^{i}\right)$ para $E$, obtemos não apenas coordenadas locais adaptadas $\left(x^{\mu}, q^{i}, q_{\mu}^{i}\right)$ para $J E$ e $\left(x^{\mu}, q^{i}, \vec{q}_{\mu}^{i}\right)$ para $\overrightarrow{J E}$, como antes, mas também coordenadas locais adaptadas

- $\left(x^{\mu}, q^{i}, p_{i}^{\mu}, p\right)$ para $J^{\star} E$ e $J^{\circledast} E$,

- $\left(x^{\mu}, q^{i}, p_{i}^{\mu}\right)$ para $\vec{J}^{*} E$ e $\vec{J}^{\circledast} E$,

de modo que, no caso afim, o pareamento entre um ponto de $J^{\star} E$ e um ponto de $J E$ é dado por

$$
p_{i}^{\mu} q_{\mu}^{i}+p
$$

e o pareamento entre um ponto de $J^{\circledast} E$ e um ponto de $J E$ é dado por

$$
\left(p_{i}^{\mu} q_{\mu}^{i}+p\right) d^{n} x
$$


enquanto que, no caso linear, o pareamento entre um ponto de $\vec{J}^{*} E$ e um ponto de $\vec{J} E$ é dado por

$$
p_{i}^{\mu} \vec{q}_{\mu}^{i}
$$

e o pareamento entre um ponto de $\vec{J} \circledast E$ e um ponto de $\vec{J} E$ é dado por

$$
p_{i}^{\mu} \vec{q}_{\mu}^{i} d^{n} x .
$$

Continuando, notamos que uma mudança de coordenadas locais adaptadas $\left(x^{\mu}, q^{i}\right) \rightarrow$ $\left(\bar{x}^{\nu}, \bar{q}^{j}\right)$ para $E$ induz não apenas mudanças de coordenadas locais adaptadas $\left(x^{\mu}, q^{i}, q_{\mu}^{i}\right) \rightarrow\left(\bar{x}^{\nu}, \bar{q}^{j}, \bar{q}_{\nu}^{j}\right)$ para $J E$ e $\left(x^{\mu}, q^{i}, \vec{q}_{\mu}^{i}\right) \rightarrow\left(\bar{x}^{\nu}, \bar{q}^{j}, \vec{q}_{\nu}^{j}\right)$ para $\overrightarrow{J E}$, como antes, mas também mudanças de coordenadas locais adaptadas

- $\left(x^{\mu}, q^{i}, p_{i}^{\mu}, p\right) \rightarrow\left(\bar{x}^{\nu}, \bar{q}^{j}, \bar{p}_{j}^{\nu}, \bar{p}\right)$ para $J^{\star} E$ e $J^{\circledast} E$,

- $\left(x^{\mu}, q^{i}, p_{i}^{\mu}\right) \rightarrow\left(\bar{x}^{\nu}, \bar{q}^{j}, \bar{p}_{j}^{\nu}\right)$ para $\overrightarrow{J^{*}} E$ e $\vec{J}^{\circledast} E$,

que são dadas por

$$
\bar{p}_{j}^{\nu}=\frac{\partial \bar{x}^{\nu}}{\partial x^{\mu}} \frac{\partial q^{i}}{\partial \bar{q}^{j}} p_{i}^{\mu}
$$

e

$$
\bar{p}=p-\frac{\partial \bar{q}^{j}}{\partial x^{\mu}} \frac{\partial q^{i}}{\partial \bar{q}^{j}} p_{i}^{\mu}
$$

para o caso comum (i.e., sem torção) e

$$
\bar{p}_{j}^{\nu}=\operatorname{det}\left(\frac{\partial x}{\partial \bar{x}}\right) \frac{\partial \bar{x}^{\nu}}{\partial x^{\mu}} \frac{\partial q^{i}}{\partial \bar{q}^{j}} p_{i}^{\mu}
$$

e

$$
\bar{p}=\operatorname{det}\left(\frac{\partial x}{\partial \bar{x}}\right)\left(p-\frac{\partial \bar{q}^{j}}{\partial x^{\mu}} \frac{\partial q^{i}}{\partial \bar{q}^{j}} p_{i}^{\mu}\right)
$$

para o caso torcido. Além disso, a projeção de $J^{\star} E$ sobre $\vec{J}^{\star} E$ e de $J^{\circledast} E$ sobre $\vec{J}{ }^{\circledast} E$ mencionada no parágrafo anterior consiste simplesmente em omitir a última coordenada (escalar) $p$.

Como próximo item, queremos apresentar a construção de uma distribuição canônica sobre o espaço total do fibrado de jatos $J E$ de qualquer fibrado $E$; uma versão da mesma para grupoides de Lie, que é de importância central para este trabalho, será apresentada no Apêndice A.

Seja $E$ um fibrado sobre uma variedade base $M$, com projeção $\pi_{E}: E \longrightarrow M$, seja $T E$ o fibrado tangente do espaço total, com projeção $\pi_{T E}: T E \longrightarrow E$, e seja $J E$ o 
fibrado dos jatos (de primeira ordem) de $E$, com projeção $\pi_{J E}: J E \longrightarrow E$ sobre $E$ e projeção $\pi_{E} \circ \pi_{J E}: J E \longrightarrow M$ sobre $M$. Para construir a desejada distribuição, que será um subfibrado vetorial canônico $H(J E)$ do fibrado tangente $T(J E)$ de $J E$, prosseguimos em dois passos.

O primeiro passo consiste em considerar o produto fibrado de $J E$ e $T E$ sobre $E$,

$$
J E \times_{E} T E=\left\{(u, v) \in J E \times T E \mid \pi_{J E}(u)=\pi_{T E}(v)\right\},
$$

que também pode ser visto como o "pull-back"

$$
J E \times_{E} T E=\pi_{J E}^{*} T E,
$$

pois isso sugere considerá-lo como fibrado vetorial sobre $J E$ que completa o seguinte diagrama:

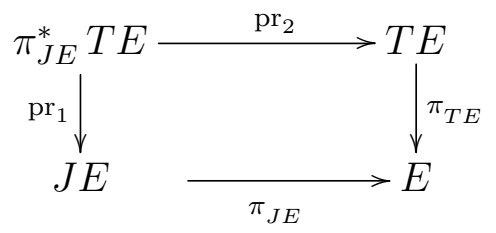

Nele, introduzimos o subfibrado vetorial $H_{J E} E$ definido por

$$
H_{J E} E=\left\{(u, v) \in J E \times_{E} T E \mid v=\left(u \circ T_{\pi_{J E}(u)} \pi_{E}\right) \cdot v\right\} .
$$

Em outras palavras, em qualquer ponto $u \in J E$ com pontos base $e=\pi_{J E}(u) \in E \mathrm{e}$ $x=\pi_{E}(e) \in M$, a fibra de $H_{J E} E$ em $u$, que a seguir denotaremos por $H_{u} E$, é simplesmente a imagem de $T_{x} M$ por $u$, como subespaço do espaço tangente $T_{e} E$ complementar ao espaço vertical $V_{e} E$, e $u \circ T_{e} \pi_{E}$ é a projeção sobre $H_{u} E$ com núcleo $V_{e} E$. Assim, percebe-se que pelo simples truque de efetuar um "pull-back" de $E$ para $J E$, obtemos uma decomposição direta canônica em parte vertical e horizontal:

$$
\pi_{J E}^{*} T E=\pi_{J E}^{*} V E \oplus H_{J E} E .
$$

Obviamente, ao contrário da parte vertical, a parte horizontal aqui não pode ser o "pullback" de algum subfibrado vetorial de $T E$, pois em $T E$ não existe tal subfibrado horizontal canônico. De fato, podemos entender melhor o significado desta construção se lembrarmos que uma conexão em $E$ pode ser definida como um subfibrado horizontal $H E$ de $T E$, complementar ao subfibrado vertical $V E$ :

$$
T E=V E \oplus H E .
$$

Equivalentemente, como já foi explicado acima, ela pode ser definida por uma seção $\Gamma: E \longrightarrow J E$, sendo que para todo $e \in E$, vale $H_{e} E=H_{\Gamma(e)} E$ e, reciprocamente, 
$\Gamma(e)=\left(\left.T_{e} \pi_{E}\right|_{H_{e} E}\right)^{-1}$. Mas isso significa que o fibrado horizontal $H E$ será simplesmente o "pull-back" de $H_{J E} E$ por $\Gamma$, e neste sentido $H_{J E} E$ seria um "fibrado horizontal universal", se não fosse o fato de que é um subfibrado não de um fibrado tangente e sim de um "pullback" de um fibrado tangente.

O segundo passo consiste em voltar a considerar subfibrados de um fibrado tangente, no caso, do fibrado tangente $T(J E)$ de $J E$. Para tanto, basta definir a distribuição $H(J E) \subset T(J E)$ como a imagem inversa de $H_{J E} E$ sob a aplicação tangente à projeção alvo, reinterpretada como morfismo estrito de fibrados vetoriais sobre $J E$, $T \pi_{J E}: T(J E) \longrightarrow \pi_{J E}^{*} T E:$

$$
H(J E)=\left(T \pi_{J E}\right)^{-1}\left[\left(H_{J E} E\right)\right] \subset T(J E) .
$$

Explicitamente, dado $u_{e} \in J_{e} E$, temos

$$
H_{u_{e}} E=\operatorname{ker}\left(\mathrm{id}_{T_{e} E}-u_{e} \circ T_{e} \pi_{E}\right) \subset T_{e} E
$$

e

$$
H_{u_{e}}(J E)=\operatorname{ker}\left(\left(\mathrm{id}_{T_{e} E}-u_{e} \circ T_{e} \pi_{E}\right) \circ T_{u_{e}} \pi_{J E}\right) \subset T_{u_{e}}(J E)
$$

ou ainda

$$
H_{u_{e}} E=\left\{v_{e} \in T_{e} E \mid v_{e}=\left(u_{e} \circ T_{e} \pi_{E}\right)\left(v_{e}\right)\right\}
$$

e

$$
H_{u_{e}}(J E)=\left\{v_{u_{e}}^{\prime} \in T_{u_{e}}(J E) \mid T_{u_{e}} \pi_{J E}\left(v_{u_{e}}^{\prime}\right)=\left(u_{e} \circ T_{u_{e}}\left(\pi_{E} \circ \pi_{J E}\right)\right)\left(v_{u_{e}}^{\prime}\right)\right\} .
$$

$H(J E)$ é chamado de distribuição de Cartan. JE possui dois subfibrados verticais: $V_{M}(J E)=\operatorname{ker} T\left(\pi_{E} \circ \pi_{J E}\right)$ e $V_{E}(J E)=\operatorname{ker} T \pi_{J E}$, sendo que o segundo é contido no primeiro. Então considerando a imagem inversa sob $T \pi_{J E}$ da decomposição direta (2.32) e usando que

$$
V_{M}(J E)=\left(T \pi_{J E}\right)^{-1}[(V E)] \subset T(J E),
$$

obtemos novamente uma decomposição canônica, mas que deixa de ser direta:

$$
T(J E)=V_{M}(J E)+H(J E) \quad, \quad V_{E}(J E)=V_{M}(J E) \cap H(J E) .
$$

Finalmente, podemos caracterizar vetores tangentes "horizontais" como tangentes a curvas "horizontais":

Definição 2.1 Uma curva $t \mapsto u(t)$ em JE é dita horizontal se a curva projetada para $E, t \mapsto e(t)=\pi_{J E}(u(t))$, é obtida da curva projetada para $M, t \mapsto x(t)=\pi_{E}(e(t))$, por levantamento horizontal pela própria curva original:

$$
\frac{d}{d t} e(t)=u(t)\left(\frac{d}{d t} x(t)\right)
$$


Então verifica-se imediatamente que uma curva $t \mapsto u(t)$ em $J E$ é horizontal se e somente se, para todo $t$, vale

$$
\frac{d}{d t} u(t) \in H_{u(t)}(J E)
$$

Por fim, queremos tecer algumas considerações sobre fibrados de jatos de ordem superior. Uma das principais possibilidades de construir estes objetos é por iteração do funtor $J$; no entanto, isso requer efetuar, posteriormente, uma "redução" para eliminar o excesso de variáveis que tal procedimento proporciona. Para uso posterior, explicamos brevemente como isso é feito no caso mais simples do fibrado dos jatos de segunda ordem. Dado um fibrado $E$ sobre uma variedade base $M$, com projeção $\pi_{E}: E \longrightarrow M$, considere o seu fibrado de jatos de primeira ordem $J E$, com projeção $\pi_{J E}: J E \longrightarrow E$, e introduza o fibrado de jatos de primeira ordem deste, $J(J E)$. O primeiro passo da redução basea-se na observação de que $J(J E)$ admite duas projeções diferentes sobre $J E$, a saber, a projeção $\pi_{J(J E)}$ construída conforme a regra anterior, substituindo $E$ por $J E$, e o "prolongamento" $J \pi_{J E}$ da projeção $\pi_{J E}$ que resulta da aplicação do funtor $J$ ao morfismo $\pi_{J E}$. De fato, como $\pi_{J E}: J E \longrightarrow E$ é um morfismo estrito de fibrados sobre $M$, esse prolongamento proporciona um novo morfismo estrito de fibrados sobre $M, J \pi_{J E}: J(J E) \longrightarrow J E$, explicitamente definido por

$$
\left(J \pi_{J E}\right)_{u_{e}}\left(u_{u_{e}}^{\prime}\right)=T_{u_{e}} \pi_{J E} \circ u_{u_{e}}^{\prime} \quad \text { para } u_{e} \in J_{e} E, u_{u_{e}}^{\prime} \in J_{u_{e}}(J E) .
$$

Podemos enquadrar ambas no seguinte diagrama comutativo, que é o análogo do "losango tangente duplo" em [1, p. 214]:

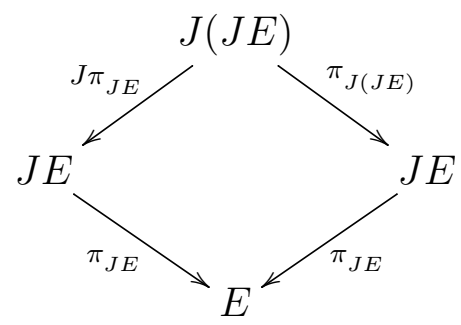

Definimos então, para cada ponto $u_{e} \in J_{e} E$,

$$
\bar{J}_{u_{e}}^{2} E=\left\{u_{u_{e}}^{\prime} \in J_{u_{e}}(J E) \mid T_{u_{e}} \pi_{J E} \circ u_{u_{e}}^{\prime}=u_{e}\right\} .
$$

Verifica-se que $\bar{J}_{u_{e}}^{2} E$ é um subespaço afim do espaço afim $J_{u_{e}}(J E)$ (que por sua vez é um subespaço afim do espaço vetorial $\left.L\left(T_{x} M, T_{u_{e}}(J E)\right)\right)$, sendo que o correspondente espaço vetorial de diferenças é dado por

$$
\overrightarrow{\vec{J}}_{u_{e}}^{2} E=\left\{\vec{u}_{u_{e}}^{\prime} \in \vec{J}_{u_{e}}(J E) \mid T_{u_{e}} \pi_{J E} \circ \vec{u}_{u_{e}}^{\prime}=0\right\} .
$$


Assim, usando que, no ponto $u_{e}$, o espaço vertical de $J E$ como fibrado sobre $E$ é o espaço tangente à fibra $J_{e} E$ e, sendo esta um espaço afim, é isomorfo ao seu espaço vetorial de diferenças $\overrightarrow{J_{e}} E=L\left(T_{x} M, V_{e} E\right)$, temos

$$
{\overrightarrow{J_{u}}}^{2} E=L^{2}\left(T_{x} M, V_{e} E\right) \cong T_{x}^{*} M \otimes T_{x}^{*} M \otimes V_{e} E
$$

Este resultado sugere, como segundo passo da redução, uma decomposição em parte simétrica e parte antissimétrica, a qual pode ser efetuada não apenas no espaço vetorial da equação (2.45) mas também no espaço afim da equação (2.43), proporcionando uma decomposição

$$
\bar{J}_{u_{e}}^{2} E=J_{u_{e}}^{2} E \oplus L_{a}^{2}\left(T_{x} M, V_{e} E\right)
$$

e uma correspondente decomposição

$$
{\overrightarrow{J_{u}}}^{2} E=L_{s}^{2}\left(T_{x} M, V_{e} E\right) \oplus L_{a}^{2}\left(T_{x} M, V_{e} E\right)
$$

onde

$$
L_{a}^{2}\left(T_{x} M, V_{e} E\right) \cong \bigwedge^{2} T_{x}^{*} M \otimes V_{e} E
$$

enquanto que $J_{u_{e}}^{2} E$ é um espaço afim, chamado de espaço dos jatos de segunda ordem em $u_{e}$, com espaço vetorial de diferenças

$$
L_{s}^{2}\left(T_{x} M, V_{e} E\right) \cong \bigvee^{2} T_{x}^{*} M \otimes V_{e} E
$$

chamado de espaço dos jatos linearizados de segunda ordem em $u_{e}$ : omitiremos os detalhes deste segundo passo, pois não serão relevantes para o que segue. O que será relevante sim são o espaço afim $\bar{J}_{u_{e}}^{2} E$, que propomos chamar de espaço dos jatos não holônomos de segunda ordem em $u_{e}$ e o seu espaço vetorial de diferenças ${\overrightarrow{J_{u}}}_{e}^{2} E$, que propomos chamar de espaço dos jatos linearizados não holônomos de segunda ordem em $u_{e}$, sendo que o termo "não holônomo" deve ser entendido como sinônimo de "não necessariamente holônomo". Variando o ponto $u_{e}$, obtemos um subfibrado afim $\bar{J}^{2} E$ do fibrado afim $J(J E)$ sobre $J E$ chamado de fibrado dos jatos não holônomos de segunda ordem de $E$ e um subfibrado vetorial $\vec{J}^{2} E$ do fibrado vetorial $\vec{J}(J E)$ chamado de fibrado dos jatos linearizados não holônomos de segunda ordem de E, com

$$
\vec{J}^{2} E \cong \pi_{J E}^{*}\left(\pi_{E}^{*}\left(T^{*} M \otimes T^{*} M\right) \otimes V E\right)
$$

Há uma estreita relação entre as duas construções, a da distribuição de Cartan e a do fibrado dos jatos não holônomos de segunda ordem: denotando por $J(J E, H(J E))$ o subfibrado de $J(J E)$ dos jatos tomando valores em $H(J E) \subset T(J E)$, temos

$$
\bar{J}^{2} E=J(J E, H(J E))
$$


A prova desta fórmula é um cálculo elementar: dados $x \in M, e \in E_{x}, u_{e} \in J_{e} E \mathrm{e}$ $u_{u_{e}}^{\prime} \in J_{u_{e}}(J E)$, vale $T_{u_{e}}\left(\pi_{E} \circ \pi_{J E}\right) \circ u_{u_{e}}^{\prime}=\mathrm{id}_{T_{x} M}$ e portanto,

$$
\begin{aligned}
& u_{u_{e}}^{\prime} \in J_{u_{e}}(J E, H(J E)) \\
& \Longleftrightarrow u_{u_{e}}^{\prime}\left(h_{x}\right) \in H_{u_{e}}(J E) \text { para todo } h_{x} \in T_{x} M \\
& \Longleftrightarrow\left(T_{u_{e}} \pi_{J E} \circ u_{u_{e}}^{\prime}\right)\left(h_{x}\right)=\left(u_{e} \circ T_{u_{e}}\left(\pi_{E} \circ \pi_{J E}\right) \circ u_{u_{e}}^{\prime}\right)\left(h_{x}\right) \text { para todo } h_{x} \in T_{x} M \\
& \Longleftrightarrow T_{u_{e}} \pi_{J E} \circ u_{u_{e}}^{\prime}=u_{e} \\
& \Longleftrightarrow u_{u_{e}}^{\prime} \in \bar{J}_{u_{e}}^{2} E .
\end{aligned}
$$

\subsection{Transformação de Legendre}

Nesta seção, apresentamos as ferramentas necessárias para tratar da relação entre a formulação hamiltoniana (covariante) e a formulação lagrangiana em teoria de campos algo conhecido já da mecânica. Tal relação é essencial, pois é muito importante que os resultados obtidos nesta tese, no âmbito hamiltoniano, estejam de acordo com resultados obtidos anteriormente [17] na abordagem lagrangiana.

Seja $E$ um fibrado sobre uma variedade base $M$. Suporemos que a dinâmica da teoria é determinada por uma lagrangiana que "depende localmente dos campos e das suas primeiras derivadas", o que significa que ela é dada por uma aplicação ${ }^{2}$

$$
\mathcal{L}: J E \longrightarrow \bigwedge^{n} T^{*} M,
$$

onde $\bigwedge^{n} T^{*} M$ é o fibrado em linhas das formas de volume sobre $M$. Esta escolha de codomínio se faz conveniente para definir o "funcional ação" $S$ no espaço $\Gamma(E)$ das seções de $E$, o qual na verdade é toda uma família de funcionais $S_{K}$ onde $K$ percorre os compactos de $M$, definidos por integração da lagrangiana $\mathcal{L}$, composta com a derivada $(\varphi, \partial \varphi) \in$ $\Gamma(J E)$ da seção $\varphi \in \Gamma(E)$ pertinente, sobre $K$ :

$$
S_{K}[\varphi]=\int_{K} \mathcal{L}(\varphi, \partial \varphi)
$$

Então o princípio variacional, ou princípio da ação estacionária, exige que os campos fisicamente admissíveis são as seções $\varphi$ de $E$ tais que, para todo compacto $K$ de $M$ suficientemente regular, ${ }^{3}$ o funcional $S_{K}$ em $\varphi$ seja estacionário sob variações $\delta \varphi$ de $\varphi$

\footnotetext{
${ }^{2}$ Mais precisamente, $\mathcal{L}$ é um morfismo estrito de fibrados sobre $M$.

${ }^{3}$ Um compacto $K$ de $M$ será chamado de regular se seu interior $K^{\circ}$ for não-trivial e suficientemente grande para que $K$ seja o fecho de $K^{\circ}$, e se o seu bordo $\partial K=K \backslash K^{\circ}$ for uma subvariedade de $M$, de modo que podemos aplicar o teorema de Stokes quando integramos $n$-formas fechadas sobre $K$.
} 
que se anulam no bordo $\partial K$ de $K$. Um argumento padrão em teoria de campos mostra que isso é equivalente a exigir que $\varphi$ satisfaça um certo sistema de equações diferenciais parciais conhecido como as equações de Euler-Lagrange.

A passagem do formalismo lagrangiano para o formalismo hamiltoniano covariante é efetuada pela transformação de Legendre covariante, que consiste de duas aplicações ${ }^{4}$

$$
\overrightarrow{\mathbb{F}} \mathcal{L}: J E \longrightarrow \vec{J}^{\circledast} E
$$

e

$$
\mathbb{F} \mathcal{L}: J E \longrightarrow J^{\circledast} E
$$

definidas como segue: temos

$$
\overrightarrow{\mathbb{F}} \mathcal{L}(u) \cdot \vec{u}^{\prime}=\left.\frac{d}{d \lambda} \mathcal{L}\left(u+\lambda \vec{u}^{\prime}\right)\right|_{\lambda=0} \quad \text { para } u \in J_{e} E, \vec{u}^{\prime} \in \vec{J}_{e} E,
$$

enquanto que

$$
\mathbb{F} \mathcal{L}(u) \cdot u^{\prime}=\mathcal{L}(u)+\left.\frac{d}{d \lambda} \mathcal{L}\left(u+\lambda\left(u^{\prime}-u\right)\right)\right|_{\lambda=0} \quad \text { para } u \in J_{e} E, u^{\prime} \in J_{e} E .
$$

Em termos de coordenadas locais adaptadas $\left(x^{\mu}, q^{i}, q_{\mu}^{i}\right)$ para $J E,\left(x^{\mu}, q^{i}, p_{i}^{\mu}\right)$ para $\vec{J}^{\circledast} E \mathrm{e}$ $\left(x^{\mu}, q^{i}, p_{i}^{\mu}, p\right)$ para $J^{\circledast} E$, podemos escrever $\mathcal{L}=L d^{n} x$ e usar as fórmulas (2.22) para o pareamento de $\vec{J}{ }^{\circledast} E$ com $\overrightarrow{J E}$ e (2.23) para o pareamento de $J^{\circledast} E$ com $J E$ para concluir que $\overrightarrow{\mathbb{F}} \mathcal{L}$ é dada por

$$
p_{i}^{\mu}=\frac{\partial L}{\partial q_{\mu}^{i}},
$$

enquanto que $\mathbb{F} \mathcal{L}$ é dada por

$$
p_{i}^{\mu}=\frac{\partial L}{\partial q_{\mu}^{i}}, \quad p=L-\frac{\partial L}{\partial q_{\mu}^{i}} q_{\mu}^{i}
$$

No âmbito do formalismo hamiltoniano covariante, a hamiltoniana do sistema é uma aplicação $^{5}$

$$
\mathcal{H}: \vec{J}^{\circledast} E \longrightarrow J^{\circledast} E
$$

\footnotetext{
${ }^{4}$ Mais precisamente, $\overrightarrow{\mathbb{F}} \mathcal{L}$ e $\mathbb{F} \mathcal{L}$ são morfismos estritos de fibrados sobre $E$.

${ }^{5}$ Mais precisamente, $\mathcal{H}$ é uma seção de $J^{\circledast} E$ como fibrado afim em linhas sobre $\vec{J}^{\circledast} E$.
} 
tal que o seguinte diagrama comuta: ${ }^{6}$

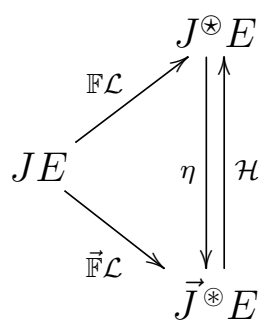

Como em [1], dizemos que o sistema é regular se $\overrightarrow{\mathbb{F}} \mathcal{L}$ for um difeomorfismo local e hiperregular se $\overrightarrow{\mathbb{F}} \mathcal{L}$ for um difeomorfismo global. Neste caso, podemos simplesmente definir a hamiltoniana $\mathcal{H}$ como a composição

$$
\mathcal{H}=\mathbb{F} \mathcal{L} \circ \overrightarrow{\mathbb{F}} \mathcal{L}^{-1}
$$

Em coordenadas locais adaptadas, como antes, e escrevendo $\mathcal{H}\left(x^{\mu}, q^{i}, p_{i}^{\mu}\right)=$ $\left(x^{\mu}, q^{i}, p_{i}^{\mu},-H\left(x^{\mu}, q^{i}, p_{i}^{\mu}\right)\right)$, recuperamos a fórmula padrão

$$
H=p_{i}^{\mu} q_{\mu}^{i}-L
$$

\subsection{Grupoides e Algebroides de Lie}

Reproduzimos nesta seção algumas noções básicas da teoria dos grupoides e algebroides de Lie, fazendo uso constante do livro [16] que, atualmente, constitui uma referência básica na área. Também abordamos alguns aspectos menos bem divulgados mas que ocupam uma posição importante para os fins desta tese, citando referências adicionais onde couber, mais notavelmente a construção do grupoide/algebroide dos jatos (de primeira ordem) de um grupoide/algebroide de Lie.

\subsubsection{Grupoides de Lie}

Começamos com uma explicação informal do que é um grupoide, para que o leitor possa desenvolver uma ideia mais intuitiva do conceito. Grosso modo, um grupoide $G$ se assemelha a um grupo, exceto pelo fato de que não é possível multiplicar quaisquer dois

\footnotetext{
${ }^{6} \mathrm{O}$ diagrama comuta no sentido de que vale $\eta \circ \mathcal{H}=\mathrm{id}_{\vec{J} \circledast E}$, mas obviamente, a composição na ordem oposta não é a identidade.
} 
elementos, sendo que ele vem munido de uma estrutura adicional que controla com precisão quando isso é ou não é possível, a saber, um espaço base $M$ e duas projeções $\sigma$ e $\tau$ de $G$ para $M$, chamadas de fonte ("source") e alvo ("target") tal que o produto de dois elementos existe se e somente se o alvo do primeiro coincide com a fonte do segundo. Sendo assim, podemos considerar os elementos $g$ de um grupoide $G$ sobre um espaço $M$ como flechas

$$
\tau(g) \longleftarrow \sigma(g)
$$

que se inciam na fonte $\sigma(g)$ e terminam no alvo $\tau(g)$. (A notação de flechas "indo da direita para a esquerda" é mera convenção.) Em relação à multiplicação, podemos compor apenas flechas tais que o alvo da primeira seja igual à fonte da segunda:

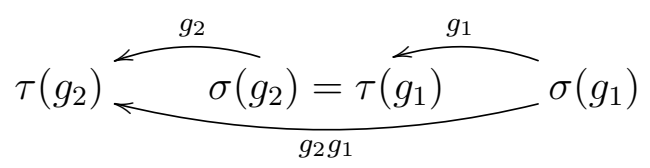

Exige-se que a multiplicação de três elementos, assim que bem definida, seja associativa:

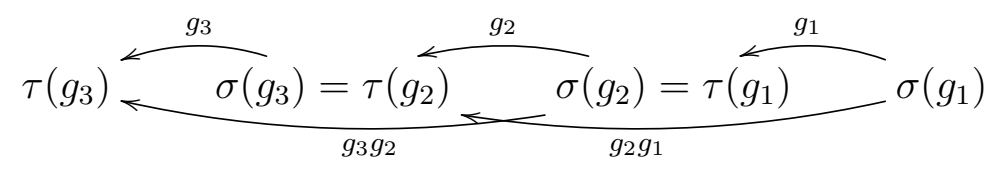

A unidade de $G$ agora não é mais um único elemento mas toda uma família de elementos, parametrizada pelos pontos do espaço base: pode ser vista como seção $1: M \longrightarrow G$, tanto em relação à projeção fonte como em relação à projeção alvo, associando a cada ponto $x$ de $M$ uma flecha que começa e termina neste ponto $x$ e de tal modo que quando multiplicarmos por outra flecha, o resultado é esta mesma outra flecha:

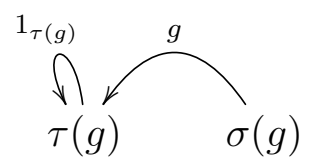

e

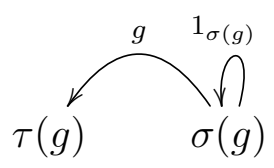

Por fim, cada flecha tem uma flecha inversa:

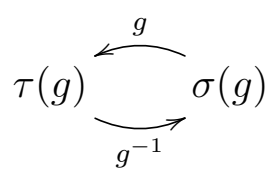


Isso resume o aspecto algébrico do que é um grupoide, mas para chegar ao conceito de um grupoide de Lie, precisamos exigir, no mínimo, que os espaços envolvidos sejam variedades diferenciáveis e as aplicações entre elas sejam diferenciáveis. Além disso, quando falamos em "projeções", devemos esperar por uma condição mais forte, tipicamente a de se tratar de submersões sobrejetoras. Assim, podemos formalizar:

Definição 2.2 Um grupoide de Lie sobre uma variedade base $M$ é uma variedade $G$ equipada com

1. duas submersões sobrejetoras $\sigma: G \longrightarrow M$, chamada de projeção fonte, e $\tau$ : $G \longrightarrow M$, chamada de projeção alvo;

2. uma aplicação diferenciável

$$
\begin{aligned}
\mu: G \times_{M} G & \longrightarrow G \\
(g, h) & \longmapsto g h
\end{aligned}
$$

chamada de multiplicação ou produto, onde

$$
G \times{ }_{M} G=\{(g, h) \in G \times G \mid \sigma(g)=\tau(h)\}
$$

tal que

$$
\sigma(g h)=\sigma(h) \quad, \quad \tau(g h)=\tau(g)
$$

e que associativa, no sentido de que vale

$$
g(h k)=(g h) k \quad \text { quando } \quad \sigma(g)=\tau(h) \quad \text { e } \sigma(h)=\tau(k) ;
$$

3. uma aplicação diferenciável

$$
\begin{aligned}
1: M & \longrightarrow G \\
x & \longmapsto 1_{x}
\end{aligned}
$$

chamada de unidade, tal que

$$
\sigma\left(1_{x}\right)=x=\tau\left(1_{x}\right)
$$

$e$

$$
g 1_{\sigma(g)}=g=1_{\tau(g)} g
$$

4. uma aplicação diferenciável

$$
\begin{aligned}
\iota: G & \longrightarrow G \\
g & \longmapsto g^{-1}
\end{aligned}
$$


chamada de inversão, tal que

$$
\sigma\left(g^{-1}\right)=\tau(g) \quad, \quad \tau\left(g^{-1}\right)=\sigma(g)
$$

$e$

$$
g^{-1} g=1_{\sigma(g)} \quad, \quad g g^{-1}=1_{\tau(g)} .
$$

$G$ é chamado espaço total e $M$ espaço base do grupoide de Lie, e ainda se adota a seguinte terminologia:

- para $x \in M, G_{x}=\sigma^{-1}(x)$ é a fibra fonte ou $\sigma$-fibra sobre $x$;

- para $y \in M,{ }_{y} G=\tau^{-1}(y)$ é a fibra alvo ou $\tau$-fibra sobre $y$;

- para $x, y \in M,{ }_{y} G_{x}=G_{x} \bigcap_{y} G$ é a fibra conjunta ou $(\tau \times \sigma)$-fibra sobre $(y, x)$;

- para $x \in M,{ }_{x} G_{x}=G_{x} \bigcap{ }_{x} G$ é o grupo de isotropia de $x$;

- para $x \in M, G \cdot x=\left\{\left.y \in M\right|_{y} G_{x} \neq \emptyset\right\}$ é a órbita de $x$ sob $G$;

e diremos que

- $G$ é transitivo se $M$ for a única órbita, i.e., para todo $x \in M, G \cdot x=M$;

- G é totalmente intransitivo se as órbitas se reduzem a pontos, i.e., para todo $x \in M, G \cdot x=\{x\}$, ou equivalentemente, se as projeções fonte e alvo coincidem.

Finalmente, a aplicação diferenciável

$$
\begin{aligned}
\tau \times \sigma: \quad G & \longrightarrow M \times M \\
g & \longmapsto(\tau(g), \sigma(g))
\end{aligned}
$$

é chamada a âncora do grupoide de Lie.

Apresentamos uma série de exemplos, começando com dois casos especiais:

Exemplo 2.1 (Grupo de Lie) Todo grupo de Lie é um grupoide de Lie sobre um ponto, e todo grupoide de Lie sobre um ponto é um grupo de Lie.

Mais geralmente, temos: 
Exemplo 2.2 (Fibrado de grupos de Lie) Todo fibrado de grupos de Lie (i.e., todo fibrado cujas fibras são grupos de Lie e que admite trivializações locais em que o produto fibra a fibra se torne constante) é um grupoide de Lie totalmente intransitivo. (A afirmação recíproca é verdadeira apenas quando se impõe alguma condição adicional de regularidade sobre o grupoide.)

Exemplo 2.3 (Grupoide de pares) Para toda variedade $M$, o produto cartesiano $M \times M$ de duas cópias de $M$ é um grupoide de Lie com projeção fonte $\sigma$ e projeção alvo $\tau$ definidas por

$$
\sigma(y, x)=x \quad, \quad \tau(y, x)=y,
$$

multiplicação definida por

$$
(z, y)(y, x)=(z, x)
$$

unidade definida por

$$
1_{x}=(x, x),
$$

e inversão definida por

$$
(y, x)^{-1}=(x, y) .
$$

Exemplo 2.4 (Grupoide de calibre) Se $P$ é um fibrado principal sobre uma variedade base $M$ com projeção $\rho$ e grupo estrutural $G_{0}$, o espaço $(P \times P) / G_{0}$ das órbitas no produto cartesiano $P \times P$ de duas cópias de $P$ pela ação "diagonal" de $G_{0}$, dada por $(q, p) \cdot g_{0}=\left(q \cdot g_{0}, p \cdot g_{0}\right)$, é um grupoide de Lie sobre $M$, com projeção fonte $\sigma$ e projeção alvo $\tau$ definidas por

$$
\sigma([q, p])=\rho(p) \quad, \quad \tau([q, p])=\rho(q)
$$

multiplicação definida por

$$
[r, q][q, p]=[r, p]
$$

unidade definida por

$$
1_{x}=[p, p]
$$

onde $p$ é qualquer ponto de $P$ tal que $\rho(p)=x$, e inversão definida por

$$
[q, p]^{-1}=[p, q]
$$

Como caso particular, podemos introduzir, para todo fibrado vetorial $E$ de posto $N$ sobre uma variedade base $M$, o grupoide das bases de $E$, denotado por $G L(E)$ : é o grupoide de calibre associado ao fibrado das bases de $E$ - um fibrado principal sobre $M$ cujo grupo estrutural é o grupo geral linear $G L(N, \mathbb{R})$. Explicitamente, suas fibras conjuntas são

$$
{ }_{y} G L(E)_{x}=G L\left(E_{x}, E_{y}\right)
$$


e as aplicações estruturais (fonte, alvo, multiplicação, unidade e inversão) são as óbvias. O mesmo vale para a multidão dos seus possíveis subgrupoides de Lie: ${ }^{7}$ a título de exemplo, mencionamos que para toda métrica pseudo-riemanniana g nas fibras de $E$, de assinatura $(r, s)$, digamos, temos o grupoide das bases ortonormais de $E$, denotado por $O(E, \mathrm{~g})$, que é o grupoide de calibre associado ao fibrado das bases ortonormais de $E$ um fibrado principal sobre $M$ cujo grupo estrutural é o grupo pseudo-ortogonal $O(r, s)$. No caso de $E$ ser o fibrado tangente $T M$ de $M$, falamos de referenciais ao invés de bases: assim, $G L(T M)$ é o grupoide dos referenciais lineares de $M$ e $O(T M, \mathrm{~g})$ é o grupoide dos referenciais ortonormais de $M$, etc.

Exemplo 2.5 (Grupoide tangente) Se $G$ é um grupoide de Lie sobre uma variedade base $M$ com projeção fonte $\sigma_{G}$ e projeção alvo $\tau_{G}$, multiplicação $\mu_{G}$, unidade $1_{G}$ e inversão $\iota_{G}$, então $T G$ é um grupoide de Lie sobre $T M$ com projeção fonte $\sigma_{T G}=T \sigma_{G}$ e projeção alvo $\tau_{T G}=T \tau_{G}$, multiplicação $\mu_{T G}=T \mu_{G}$ (mediante o isomorfismo canônico $\left.T G \times_{T M} T G \cong T\left(G \times_{M} G\right)\right)$, unidade $1_{T G}=T 1_{G}$ e inversão $\iota_{T G}=T \iota_{G}$.

Para uma melhor compreensão do próximo exemplo, precisamos introduzir uma ligeira modificação do conceito do espaço dos jatos apresentado na Seção 2.1 que se faz necessária quando tratamos de grupoides (com duas projeções) ao invés de fibrados (com apenas uma projeção): isso nos leva a substituir a definição dada pela equação (2.2) pela seguinte: se $G$ é um grupoide de Lie sobre uma variedade base $M$ com projeção fonte $\sigma$ e projeção alvo $\tau$, então para cada ponto $g$ do espaço total $G$, com pontos base $x=\sigma(g)$ e $y=\tau(g)$, define-se o espaço dos jatos (de primeira ordem) em $g$ como o conjunto dos subespaços de $T_{g} G$ que são horizontais em relação a ambas as projeções, i.e.,

$$
J_{g}^{h} G=\left\{H_{g} G \text { subespaço de } T_{g} G \mid T_{g} G=V_{g}^{\sigma} G \oplus H_{g} G \text { e } T_{g} G=V_{g}^{\tau} G \oplus H_{g} G\right\},
$$

onde

$$
V_{g}^{\sigma} G=\operatorname{ker} T_{g} \sigma \quad \text { e } \quad V_{g}^{\tau} G=\operatorname{ker} T_{g} \tau
$$

são os espaços verticais de $G$ em $g$. Novamente, podemos reformular esta definição em termos mais práticos, só que agora de duas formas diferentes:

$$
J_{g}^{\sigma} G=\left\{u_{g}^{\sigma} \in L\left(T_{x} M, T_{g} G\right) \mid T_{g} \sigma \circ u_{g}^{\sigma}=\operatorname{id}_{T_{x} M} \text { e } T_{g} \tau \circ u_{g}^{\sigma} \in G L\left(T_{x} M, T_{y} M\right)\right\}
$$

ou

$$
J_{g}^{\tau} G=\left\{u_{g}^{\tau} \in L\left(T_{y} M, T_{g} G\right) \mid T_{g} \sigma \circ u_{g}^{\tau} \in G L\left(T_{y} M, T_{x} M\right) \text { e } T_{g} \tau \circ u_{g}^{\tau}=\operatorname{id}_{T_{y} M}\right\}
$$

\footnotetext{
${ }^{7}$ Uma definição formal do conceito de subgrupoide de Lie será apresentada logo abaixo.
} 
onde a relação entre as três formas é explicitamente dada pelo seguinte diagrama comutativo

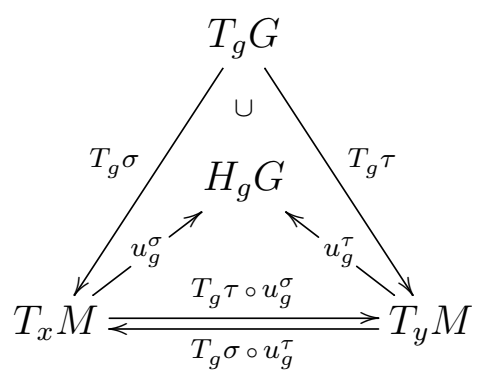

em conjunto com o fato de que

$$
\operatorname{im} u_{g}^{\sigma}=H_{g} G=\operatorname{im} u_{g}^{\tau} .
$$

Podemos chamar $u_{g}^{\sigma}$ o representante fonte e $u_{g}^{\tau}$ o representante alvo do jato, e trabalhar com ambos sem dúvida simplificaria algumas fórmulas na área, mas infelizmente tal convenção não foi adotada pela comunidade - motivo pelo qual seguiremos o procedimento padrão de trabalhar exclusivamente com os representantes fonte e omitir o índice $\sigma$, de modo que (2.62) acaba sendo simplificado para

$$
J_{g} G=\left\{u_{g} \in L\left(T_{x} M, T_{g} G\right) \mid T_{g} \sigma \circ u_{g}=\mathrm{id}_{T_{x} M} \text { e } T_{g} \tau \circ u_{g} \in G L\left(T_{x} M, T_{y} M\right)\right\} .
$$

De qualquer forma, fica claro que $J_{g} G$ é um subconjunto aberto e denso de um espaço afim, do mesmo tipo das matrizes não-singulares que formam um subconjunto aberto e denso do espaço de todas as matrizes quadradas.

Exemplo 2.6 (Grupoide dos jatos) Se $G$ é um grupoide de Lie sobre uma variedade base $M$ com projeção fonte $\sigma_{G}$ e projeção alvo $\tau_{G}$, multiplicação $\mu_{G}$, unidade $1_{G}$ e inversão $\iota_{G}$, então $J G$ é novamente um grupoide de Lie sobre $M$ com projeção fonte $\sigma_{J G} \mathrm{e}$ projeção alvo $\tau_{J G}$ definidas por

$$
\sigma_{J G}\left(u_{g}\right)=\sigma(g), \tau_{J G}\left(u_{g}\right)=\tau(g),
$$

multiplicação definida por

$$
\mu_{J G}\left(v_{h}, u_{g}\right)=T_{(h, g)} \mu_{G} \circ\left(v_{h} \circ\left(T_{g} \tau \circ u_{g}\right), u_{g}\right),
$$

unidade definida por

$$
\left(1_{J G}\right)_{x}=T_{x} 1_{G}
$$

e inversão definida por

$$
\iota_{J G}\left(u_{g}\right)=T_{g} \iota_{G} \circ u_{g} \circ\left(T_{g} \tau \circ u_{g}\right)^{-1} .
$$


O exemplo final a ser discutido aqui requer, preliminarmente, introduzir uma outra noção de fundamental importância na área de grupoides: a de ação.

Definição 2.3 Seja $G$ um grupoide de Lie sobre uma variedade base $M$, com projeção fonte $\sigma$ e projeção alvo $\tau$, e seja $E$ um fibrado sobre a mesma variedade base $M$, com projeção $\pi$. Uma ação de $G$ sobre $E$ é uma aplicação diferenciável

$$
\begin{aligned}
\Phi_{E}: G \times_{M} E & \longrightarrow E \\
(g, e) & \longmapsto g \cdot e
\end{aligned}
$$

onde $G \times_{M} E$ é o produto fibrado de $G$ (em relação à projeção fonte) e $E$,

$$
G \times{ }_{M} E=\{(g, e) \in G \times G \mid \sigma(g)=\pi(e)\},
$$

tal que

$$
\pi(g \cdot e)=\tau(g)
$$

satisfazendo

$$
h \cdot(g \cdot e)=(h g) \cdot e \quad \text { quando } \quad \sigma(h)=\tau(g) \text { e } \sigma(g)=\pi(e),
$$

$e$

$$
1_{\pi(e)} \cdot e=e .
$$

Exemplo 2.7 (Grupoide de ação) Se $E$ é um fibrado sobre uma variedade base $M$ com projeção $\pi$, munido de uma ação de um grupoide de Lie $G$ sobre a mesma variedade base $M$, com projeção fonte $\sigma_{G}$ e projeção alvo $\tau_{G}$, multiplicação $\mu_{G}$, unidade $1_{G}$ e inversão $\iota_{G}$, o produto fibrado $G \times_{M} E$ pode ser munido da estrutura de um grupoide de Lie sobre o espaço total $E$ do fibrado original, que a seguir será denotado por $G \varangle E$, com projeção fonte $\sigma_{G \varangle E}$ e projeção alvo $\tau_{G \varangle E}$ definidas por

$$
\begin{aligned}
\sigma_{G \varangle E}=\mathrm{pr}_{2}: & G \varangle E \\
(g, e) & \longmapsto E
\end{aligned}
$$

e

$$
\begin{aligned}
& \tau_{G \varangle E}=\Phi_{E}: \quad G \varangle E \quad \longrightarrow \quad E \\
& (g, e) \longmapsto g \cdot e
\end{aligned}
$$

multiplicação $\mu_{G \varangle E}$ definida por

$$
\begin{array}{rlc}
\mu_{G \varangle E}:(G \varangle E) \times_{E}(G \varangle E) & \longrightarrow & G \varangle E \\
\left(\left(g_{2}, e_{2}\right),\left(g_{1}, e_{1}\right)\right) & \longmapsto & \left(g_{2}, e_{2}\right)\left(g_{1}, e_{1}\right)=\left(g_{2} g_{1}, e_{1}\right)
\end{array}
$$


onde notamos que

$$
(G \varangle E) \times_{E}(G \varangle E)=\left\{\left(\left(g_{2}, e_{2}\right),\left(g_{1}, e_{1}\right)\right) \mid e_{2}=g_{1} \cdot e_{1}\right\},
$$

unidade $1_{G \varangle E}$ definida por

$$
\begin{array}{ccc}
1_{G \varangle E}: E & \longrightarrow & G \varangle E \\
e & \longmapsto & \left(1_{G \varangle E}\right)_{e}=\left(\left(1_{G}\right)_{\pi(e)}, e\right)
\end{array}
$$

e inversão $\iota_{G \varangle E}$ definida por

$$
\begin{array}{rlc}
\iota_{G \varangle E}: G \varangle E & \longrightarrow & G \varangle E \\
(g, e) & \longmapsto & (g, e)^{-1}=\left(g^{-1}, g \cdot e\right)
\end{array}
$$

Seguiremos com uma breve apresentação dos conceitos de morfismo entre grupoides, de subgrupoide e de produto fibrado de grupoides, todos já adaptados ao caso de grupoides de Lie (embora possam ser definidos de modo mais geral). Para um tratamento mais detalhado sugerimos consultar [16].

Definição 2.4 Sejam G um grupoide de Lie sobre uma variedade base $M$ e $H$ um grupoide de Lie sobre uma variedade base $N$, com respectivas projeções fonte $\sigma_{G}$ e $\sigma_{H}$ e projeções alvo $\tau_{G}$ e $\tau_{H}$. Um morfismo de grupoides de Lie, de $G$ em H, é uma aplicação diferenciável $F: G \longrightarrow H$ que preserva as fibras fonte e as fibras alvo, no sentido de que existe uma (única) aplicação diferenciável $f: M \longrightarrow N$ que satisfaz

$$
\sigma_{H} \circ F=f \circ \sigma_{G} \quad \text { e } \tau_{H} \circ F=f \circ \tau_{G},
$$

o que expressamos pela afirmação de que o diagrama

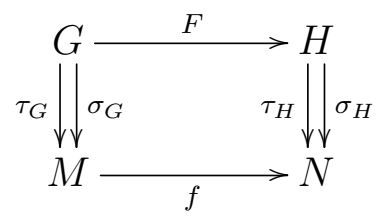

comuta, e que também preserva produtos: para todo $\left(g_{2}, g_{1}\right) \in G \times_{M} G$, vale

$$
F\left(g_{2} g_{1}\right)=F\left(g_{2}\right) F\left(g_{1}\right) .
$$

Neste caso, dizemos que F é um morfismo sobre $f$ ou que $F$ induz $f$. Se $M=N$ e $f$ for a identidade, dizemos que $F$ é um morfismo estrito.

As noções de endomorfismo, isomorfismo e automorfismo são definidos da maneira usual. 
Exemplo 2.8 Se $G$ é um grupoide de Lie sobre uma variedade base $M$ com projeção fonte $\sigma$ e projeção alvo $\tau$, então a âncora $\tau \times \sigma$ é um morfismo estrito de grupoides de Lie de $G$ no grupoide de pares $M \times M$ :

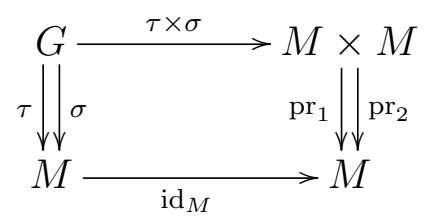

Exemplo 2.9 Se $G$ é um grupoide de Lie sobre uma variedade base $M$, com projeção fonte $\sigma_{G}$ e projeção alvo $\tau_{G}$, e $T G$ é seu grupoide tangente sobre $T M$, com projeção fonte $\sigma_{T G}=T \sigma_{G}$ e projeção alvo $\tau_{T G}=T \tau_{G}$, então a projeção tangente $\pi_{T G}$ é um morfismo de grupoides de Lie sobre a projeção tangente $\pi_{T M}$ :

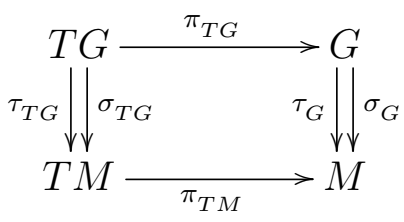

Exemplo 2.10 Se $G$ é um grupoide de Lie sobre uma variedade base $M$, com projeção fonte $\sigma_{G}$ e projeção alvo $\tau_{G}$, e $J G$ é seu grupoide dos jatos sobre $M$, com projeção fonte $\sigma_{J G}$ e projeção alvo $\tau_{J G}$, então a projeção $\pi_{J G}$ de $J G$ sobre $G$ é, por construção, um morfismo estrito de grupoides de Lie sobre $M$ :

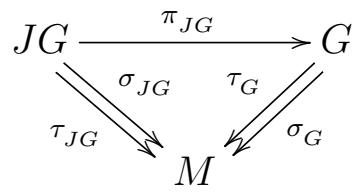

Exemplo 2.11 Se $E$ é um fibrado sobre uma variedade base $M$ com projeção $\pi$, munido de uma ação de um grupoide de Lie $G$ sobre a mesma variedade base $M$, com projeção fonte $\sigma_{G}$ e projeção alvo $\tau_{G}$, então a primeira projeção $\mathrm{pr}_{1}$ é um morfismo de grupoides de Lie do grupoide de ação $G \varangle E$ no grupoide original $G$ :

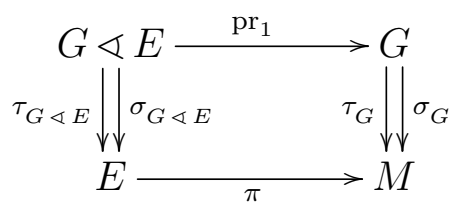

Como caso especial, temos a noção de subgrupoide de Lie: 
Definição 2.5 Sejam $G$ um grupoide de Lie sobre uma variedade base $M$ e $H$ um grupoide de Lie sobre uma variedade base $N$ tal que $H \subset G$ e $N \subset M$. Dizemos que $H$ é um subgrupoide de Lie de $G$ se a inclusão $H \hookrightarrow G$ for um morfismo de grupoides de Lie sobre a inclusão $N \hookrightarrow M$. Se $M=N$, dizemos que $H$ é um subgrupoide amplo de $G$. E se $H$ for subvariedade mergulhada de $G$ e $N$ for subvariedade mergulhada de $M$, dizemos que $H$ é um subgrupoide mergulhado de $G$.

Finalmente, precisaremos de um método para combinar dois grupoides sobre a mesma base em um novo, que podemos chamar de produto fibrado, análogo ao produto fibrado de dois fibrados. Dados grupoides de Lie $G$ e $H$, ambos sobre a mesma variedade base $M$, com respectivas projeções fonte $\sigma_{G}$ e $\sigma_{H}$ e projeções alvo $\tau_{G}$ e $\tau_{H}$, definimos o produto fibrado de $G$ e $H$ como sendo

$$
G \times_{M} H=\left\{(g, h) \in G \times H \mid \sigma_{G}(g)=\sigma_{H}(h) \text { e } \tau_{G}(g)=\tau_{H}(h)\right\} .
$$

Este produto fibrado é um grupoide de Lie sobre $M$ com projeção fonte $\sigma_{G \times_{M} H}$ e projeção alvo $\tau_{G \times_{M} H}$ definidas por

$$
\sigma_{G \times_{M} H}(g, h)=\sigma_{G}(g)=\sigma_{H}(h) \quad, \quad \tau_{G \times_{M} H}(g, h)=\tau_{G}(g)=\tau_{H}(h),
$$

multiplicação definida por

$$
\left(g_{2}, h_{2}\right)\left(g_{1}, h_{1}\right)=\left(g_{2} g_{1}, h_{2} h_{1}\right),
$$

unidade definida por

$$
\left(1_{G \times_{M} H}\right)_{x}=\left(\left(1_{G}\right)_{x},\left(1_{H}\right)_{x}\right)
$$

e inversão definida por

$$
(g, h)^{-1}=\left(g^{-1}, h^{-1}\right) .
$$

Combinando algumas das construções anteriores, obtemos um exemplo interessante de um morfismo estrito entre grupoides de Lie:

Exemplo 2.12 Se $G$ é um grupoide de Lie sobre uma variedade base $M, J G$ é seu grupoide dos jatos (Exemplo 2.6) e $G L(T M)$ é o grupoide dos referenciais lineares de $M$, então a aplicação

$$
\begin{aligned}
J G & \longrightarrow G L(T M) \times_{M} G \\
u_{g} & \longmapsto\left(T_{g} \tau \circ u_{g}, g\right)
\end{aligned}
$$

é um morfismo estrito de grupoides de Lie.

Finalmente, discutimos o análogo do conceito de seções de fibrados para grupoides, que é o de bisseções: 
Definição 2.6 Seja $G$ um grupoide de Lie sobre uma variedade base $M$ com projeção fonte $\sigma$ e projeção alvo $\tau$. Uma bisseção de $G$ é uma aplicação $\beta: M \longrightarrow G$ que satisfaz $\sigma \circ \beta=\operatorname{id}_{M}$ e $\tau \circ \beta \in \operatorname{Diff}(M)$.

O conjunto de todas as bisseções de $G$ será denotado por $\operatorname{Bis}(G)$ e forma um grupo, com produto dado por multiplicação pontual (mediante o devido acerto dos pontos no domínio),

$$
\left(\beta_{2} \beta_{1}\right)(x)=\beta_{2}\left(\tau\left(\beta_{1}(x)\right)\right) \beta_{1}(x) \quad \text { para } x \in M
$$

de modo que

$$
\tau \circ\left(\beta_{2} \beta_{1}\right)=\left(\tau \circ \beta_{2}\right) \circ\left(\tau \circ \beta_{1}\right),
$$

com unidade dada pela bisseção

$$
\begin{aligned}
1: M & \longrightarrow G \\
x & \longmapsto 1_{x}
\end{aligned}
$$

e com inversão dada por

$$
\beta^{-1}(x)=\left(\beta\left((\tau \circ \beta)^{-1}(x)\right)\right)^{-1} \quad \text { para } x \in M .
$$

Para uso posterior, notamos o seguinte homomorfismo canônico de grupos:

$$
\begin{array}{ccc}
\operatorname{Bis}(G) & \longrightarrow \operatorname{Diff}(M) \\
\beta & \longmapsto & \tau \circ \beta
\end{array}
$$

Um caso especial importante ocorre quando o grupoide de Lie provém de um fibrado de grupos de Lie e, portanto, é totalmente intransitivo, pois as projeções fonte e alvo coincidem com a projeção de fibrado. Obviamente, isso implica que bisseções de um tal grupoide de Lie correspondem a seções deste fibrado de grupos de Lie no sentido usual, sendo que o homomorfismo (2.70) se torna trivial. Tais grupoides de Lie descrevem simetrias internas, ou simetrias de calibre.

Agora podemos também entender como uma ação de um grupoide de Lie sobre um fibrado induz uma representação do seu grupo de bisseções por automorfismos desse fibrado. Para tanto, é conveniente também fazer uso do correspondente grupoide de ação e, ainda, do seu grupo de bisseções. Mantendo as notações anteriores, suponha então que $E$ seja um fibrado sobre uma variedade base $M$ com projeção $\pi$, munido de uma ação de um grupoide de Lie $G$ sobre a mesma variedade base $M$, com projeção fonte $\sigma_{G}$ e projeção alvo $\tau_{G}$, de modo que podemos formar o grupoide de ação $G \varangle E$, com projeção fonte $\sigma_{G \varangle E}$ e projeção alvo $\tau_{G \varangle E}$, como no Exemplo 2.7, e considerar os dois grupos de 
bisseções $\operatorname{Bis}(G)$ e $\operatorname{Bis}(G \varangle E)$. Então podemos construir um homomorfismo canônico de grupos

$$
\begin{array}{ccc}
\operatorname{Bis}(G) & \longrightarrow & \operatorname{Bis}(G \varangle E) \\
\beta & \longmapsto & \beta^{E}
\end{array}
$$

da seguinte forma:

$$
\beta^{E}=\left(\beta \circ \pi, \operatorname{id}_{E}\right) .
$$

Verifica-se que $\beta^{E}$ é de fato uma bisseção, pois a composição com $\sigma_{G \varangle E}=\mathrm{pr}_{2}$ é trivialmente a identidade de $E$, enquanto que a composição com $\tau_{G \varangle E}=\Phi_{E}$ é um difeomorfismo de $E$ : na verdade, é mais do que isso, pois é um automorfismo de $E$ sobre $\tau_{G} \circ \beta \in \operatorname{Diff}(M)$. De fato, temos

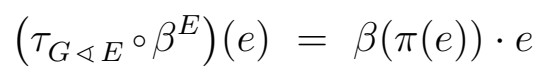

e portanto, para todo ponto $x$ de $M$, a restrição de $\tau_{G \varangle E} \circ \beta^{E}$ à fibra $E_{x}$ é o difeomorfismo, de $E_{x}$ para $E_{y}$ onde $y=\tau_{G}(\beta(x))$ (lembrando que $x=\sigma_{G}(\beta(x))$ ), de translação à esquerda por $\beta(x) \in{ }_{y} G_{x}$, mediante a ação $\Phi_{E}$. Também é elementar provar que a aplicação (2.71), além de bem definida, é um homomorfismo de grupos: para $\beta_{1}, \beta_{2} \in$ $\operatorname{Bis}(G)$ e $e \in E \operatorname{com} \pi(e)=x$, temos

$$
\begin{aligned}
\left(\beta_{2}^{E} \beta_{1}^{E}\right)(e) & =\beta_{2}^{E}\left(\tau_{G \varangle E}\left(\beta_{1}^{E}(e)\right)\right) \beta_{1}^{E}(e)=\beta_{2}^{E}\left(\beta_{1}(x) \cdot e\right) \beta_{1}^{E}(e) \\
& =\left(\beta_{2}\left(\tau_{G}\left(\beta_{1}(x)\right)\right), \beta_{1}(x) \cdot e\right)\left(\beta_{1}(x), e\right) \\
& =\left(\beta_{2}\left(\tau_{G}\left(\beta_{1}(x)\right)\right) \beta_{1}(x), e\right)=\left(\left(\beta_{2} \beta_{1}\right)(x), e\right) \\
& =\left(\beta_{2} \beta_{1}\right)^{E}(e) .
\end{aligned}
$$

Por outro lado, conforme a equação (2.70), também temos um homomorfismo canônico de grupos

$$
\begin{array}{cl}
\operatorname{Bis}(G \varangle E) & \longrightarrow \operatorname{Diff}(E) \\
\eta & \longmapsto \tau_{G \varangle E}{ }^{\circ} \eta
\end{array}
$$

que é simplesmente o "push-forward" com a ação $\Phi_{E}: G \times_{M} E \longrightarrow E$. Compondo, obtemos um homomorfismo canônico de grupos

$$
\begin{array}{rlc}
\Pi_{E}: \operatorname{Bis}(G) & \longrightarrow & \operatorname{Aut}(E) \\
\beta & \longmapsto \Pi_{E}(\beta)=\Phi_{E} \circ\left(\beta \circ \pi, \operatorname{id}_{E}\right)
\end{array}
$$

onde $\operatorname{Aut}(E)$ denota o grupo de automorfismos de $E$ : é a representação do grupo das bisseções de $G$ por automorfismos de $E$ induzida pela ação dada de $G$ sobre $E$. Nota-se 
então que o diagrama

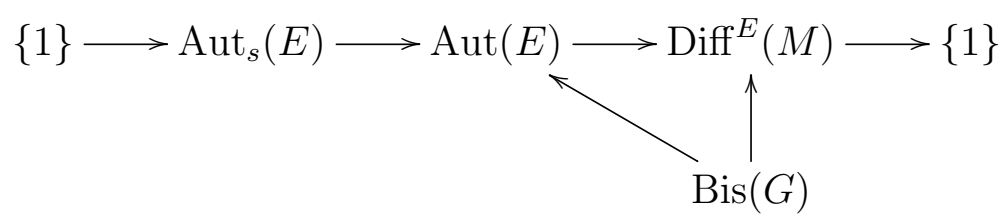

comuta, onde $\operatorname{Aut}_{s}(E)$ denota o grupo de automorfismos estritos de $E$ e $\operatorname{Diff}^{E}(M)$ denota o grupo de difeomorfismos de $M$ que admitem algum levantamento para um automorfismo de $E$.

\subsubsection{Algebroides de Lie}

O conceito de algebroide de Lie aparece naturalmente como a "contrapartida infinitesimal" de grupoide de Lie. Nesta subseção, apresentamos primeiro a definição formal. Em seguida, construímos o algebroide de Lie de um grupoide de Lie e discutimos alguns exemplos importantes para o contexto desta tese.

Definição 2.7 Um algebroide de Lie sobre uma variedade base $M$ é um fibrado vetorial A sobre $M$ munido de um morfismo estrito de fibrados vetoriais

$$
\alpha: A \longrightarrow T M
$$

chamado de âncora, e uma aplicação $\mathbb{R}$-bilinear antissimétrica

$$
[., .]: \Gamma(A) \times \Gamma(A) \longrightarrow \Gamma(A)
$$

que satisfaz a identidade de Jacobi, chamada de colchete, tal que vale a seguinte regra de Leibniz

$$
[X, f Y]=f[X, Y]+\left(L_{\alpha(X)} f\right) Y
$$

para qualquer função $f \in C^{\infty}(M)$ e quaisquer seções $X, Y \in \Gamma(A)$.

Diremos que

- A é transitivo se a for sobrejetor;

- A é totalmente intransitivo se $\alpha=0$.

Observação 2.1 Aqui e a seguir, sempre usaremos o mesmo símbolo $\alpha$ para denotar tanto o morfismo de fibrados vetoriais (2.76) quanto o homomorfismo de álgebras de Lie $\alpha: \Gamma(A) \longrightarrow \mathfrak{X}(M)$ obtido a partir dele por "push-forward" de seções. 
Da mesma forma que o colchete em uma álgebra de Lie $\mathfrak{g}$ pode, mediante a escolha de uma base de geradores $T_{a}$, ser expresso em termos de constantes de estrutura $f_{a b}^{c}$, conforme a fórmula

$$
\left[T_{a}, T_{b}\right]=f_{a b}^{c} T_{c},
$$

podemos também expressar a âncora e o colchete em um algebroide de Lie $A$, mediante a escolha de coordenadas locais $x^{\mu}$ para $M$ e uma base de seções locais $T_{a}$ de $A$, em termos de funções de estrutura (de dois tipos, um representando a âncora e outro representando o colchete), $f_{a}^{\mu}$ e $f_{a b}^{c}$, conforme as fórmulas

$$
\alpha\left(T_{a}\right)=f_{a}^{\mu} \partial_{\mu}, \quad\left[T_{a}, T_{b}\right]=f_{a b}^{c} T_{c} .
$$

Trataremos agora da construção do algebroide de Lie de um grupoide de Lie, que é análoga à de álgebra de Lie de um grupo de Lie. A principal diferença é que enquanto a álgebra de Lie de um grupo de Lie pode ser definida como sendo seu espaço tangente na unidade, o algebroide de Lie de um grupoide de Lie deve de algum modo ser visto como a união de vários espaços tangentes, já que grupoides de Lie possuem várias unidades (uma para cada ponto na variedade base); mais exatamente, deve ser um fibrado vetorial sobre a correspondente variedade base. Uma interpretação diferente, porém equivalente, usa campos vetoriais invariantes, o que permite deduzir o colchete a partir do colchete de Lie entre campos vetoriais, mas novamente há algumas diferenças importantes: enquanto para grupos de Lie, a definição de um campo vetorial invariante (digamos, à direita) é óbvia, é essencial levar em conta que em grupoides de Lie, a translação à direita por um elemento é definida somente ao longo da correspondente fibra fonte.

Explicitamente, a construção é a seguinte: dado um grupoide de Lie $G$ sobre uma variedade base $M$ com projeção fonte $\sigma$ e projeção alvo $\tau$, consideramos $M$ como subvariedade mergulhada de $G$ através da unidade $1: M \longrightarrow G$, e restringimos as projeções fonte e alvo do grupoide tangente a esta subvariedade para obter morfismos estritos de fibrados vetoriais sobre $M$ que podemos colocar no seguinte diagrama:

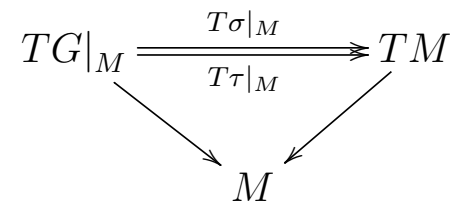

Então definimos o correspondente algebroide de Lie $A G$, primeiro como fibrado vetorial sobre $M$, por restrição ao fibrado vertical em relação à projeção fonte:

$$
A G=\left.\left(V^{\sigma} G\right)\right|_{M}=\left.\operatorname{ker} T \sigma\right|_{M} .
$$


Assim, temos para todo $x \in M$,

$$
(A G)_{x}=V_{1_{x}}^{\sigma} G=T_{1_{x}}\left(G_{x}\right) .
$$

A âncora de $A G$ é definida por

$$
\alpha=\left.\left(\left.T \tau\right|_{M}\right)\right|_{A G} .
$$

Para definir o colchete entre seções de $A G$, notamos primeiro que dado qualquer elemento $g$ em $G$, com $x=\sigma(g)$ e $y=\tau(g)$, a translação à direita por $g$ é definida apenas como um difeomorfismo da $\sigma$-fibra $G_{y}$ para a $\sigma$-fibra $G_{x}$ :

$$
\begin{aligned}
R_{g}: G_{y} & \longrightarrow G_{x} \\
h & \longmapsto h g
\end{aligned}
$$

Diferenciando em $h \in G_{y}$, obtemos um isomorfismo linear $T_{h} R_{g}: T_{h}\left(G_{y}\right) \longrightarrow T_{h g}\left(G_{x}\right)$. Assim, dado um campo vetorial $Z$ sobre $G$ que é $\sigma$-vertical, $Z \in \Gamma\left(V^{\sigma} G\right)$, podemos dizer que $Z$ é invariante à direita se, para todo $(h, g) \in G \times{ }_{M} G$, vale

$$
T_{h} R_{g}(Z(h))=Z(h g) .
$$

Seguindo o mesmo caminho que na construção da álgebra de Lie de um grupo de Lie, verificamos então que (a) o espaço $\mathfrak{X}^{r i}(G)$ dos campos vetoriais sobre $G$ invariantes à direita é uma subálgebra de Lie de $\mathfrak{X}(G)$ e (b) restrição a $M$ estabelece um isomorfismo linear do espaço $\mathfrak{X}^{r i}(G)$ para o espaço $\Gamma(A G)$ das seções de $A G$ cujo inverso podemos descrever assim: dada uma seção $X \in \Gamma(A G)$, o correspondente campo $X^{r} \in \mathfrak{X}^{r i}(G)$ é definido por

$$
X^{r}(g)=T_{1_{\tau(g)}} R_{g}(X(\tau(g))) .
$$

Este isomorfismo linear pode então ser empregado para transferir a estrutura de álgebra de Lie de $\mathfrak{X}^{r i}(G)$ para $\Gamma(A G)$.

Uma ferramenta importante que se estende naturalmente da teoria dos grupos de Lie para a dos grupoides de Lie é a exponencial, que agora será uma aplicação

$$
\exp : \Gamma(A G) \longrightarrow \operatorname{Bis}(G)
$$

definida por

$$
\exp (X)(x)=F_{X^{r}}\left(1,1_{x}\right) \quad \text { para } x \in M
$$

onde $F_{X^{r}}$ é o fluxo do campo vetorial $X^{r}$ sobre $G$ invariante à direita que corresponde a $X{ }^{8}$ Mais geralmente, vale

$$
F_{X^{r}}(t, g)=\exp (t X)(y) g \quad \text { para } t \in \mathbb{R}, g \in{ }_{y} G_{x},
$$

\footnotetext{
${ }^{8}$ Para simplificar a apresentação, estamos desconsiderando questões de domínio que aparecem quando a âncora $\alpha(X)$ de $X$ for um campo vetorial sobre $M$ que não é completo. Tais problemas não aparecem no caso totalmente intransitivo $(\alpha=0)$ ou quando a variedade base $M$ for compacta.
} 
e reciprocamente,

$$
\left.\exp (t X)(x)\right|_{t=0}=1_{x} \quad,\left.\quad \frac{d}{d t} \exp (t X)(x)\right|_{t=0}=X(x) \quad \text { para } x \in M .
$$

Apresentamos agora uma série de exemplos de algebroides de Lie, análogos aos exemplos de grupoides de Lie discutidos anteriormente, começando com dois casos especiais:

Exemplo 2.13 (Álgebra de Lie) Toda álgebra de Lie é um algebroide de Lie sobre um ponto, e todo algebroide de Lie sobre um ponto é uma álgebra de Lie. A construção do algebroide de Lie associado a um grupoide de Lie, neste caso, se reduz à construção padrão da álgebra de Lie associada a um grupo de Lie.

Mais geralmente, temos

Exemplo 2.14 (Fibrado de álgebras de Lie) Todo fibrado de álgebras de Lie (i.e., todo fibrado vetorial cujas fibras são álgebras de Lie e que admite trivializações locais em que o comutador fibra a fibra se torne constante) é um algebroide de Lie totalmente intransitivo. (A afirmação recíproca é verdadeira apenas quando se impõe alguma condição adicional de regularidade sobre o algebroide.) A construção do algebroide de Lie associado a um grupoide de Lie, neste caso, se reduz à construção padrão da álgebra de Lie associada a um grupo de Lie, fibra a fibra.

Exemplo 2.15 (Fibrado tangente) Para toda variedade $M$, o seu fibrado tangente $T M$ é um algebroide de Lie com âncora $\alpha=\mathrm{id}_{T M}$ e colchete dado pelo colchete de Lie entre campos vetoriais sobre $M$. A menos de isomorfismo, este é o algebroide de Lie associado ao grupoide de pares.

Exemplo 2.16 (Algebroide de calibre) Se $P$ é um fibrado principal sobre uma variedade base $M$ com projeção $\rho$ e grupo estrutural $G_{0}$, o espaço $T P / G_{0}$ das órbitas no fibrado tangente $T P$ de $P$ pela ação induzida de $G_{0}$ é um algebroide de Lie sobre $M$, com âncora $\alpha: T P / G_{0} \longrightarrow T M$ induzida pela aplicação tangente $T \rho: T P \longrightarrow T M$ à projeção $\rho$ e colchete dado pelo colchete de Lie entre campos vetoriais sobre $P$, mediante passagem ao quociente. Também é chamado de fibrado de Atiyah pois faz parte da sequência de Atiyah, que é a seguinte sequência exata de fibrados vetoriais sobre $M$ :

$$
0 \longrightarrow P \times_{G} \mathfrak{g} \longrightarrow T P / G_{0} \longrightarrow T M \longrightarrow 0 .
$$

A menos de isomorfismo, este é o algebroide de Lie associado ao grupoide de calibre. 
Como caso particular, podemos introduzir, para todo fibrado vetorial $E$ de posto $N$ sobre uma variedade base $M$, o algebroide das bases de $E$, denotado por $\mathfrak{g l}(E)$ : é o algebroide de calibre associado ao fibrado das bases de $E$. Uma construção explícita do fibrado vetorial subjacente diretamente em termos do próprio fibrado vetorial $E$, em vez do fibrado principal das bases de $E$, parece ser complicada e para fins deste trabalho não será necessária, mas o que é possível é dar uma definição muito elegante do seu espaço de seções: é o espaço dos campos vetoriais sobre o espaço total que comutam, sob a derivada de Lie, com o campo de Euler $\Sigma$ :

$$
\Gamma(\mathfrak{g l}(E))=\{X \in \mathfrak{X}(E) \mid[\Sigma, X]=0\} .
$$

Nota-se que campos vetoriais sobre $E$ que comutam com o campo de Euler são automaticamente projetáveis, o que torna óbvio qual é a definição da âncora (em nível de seções) e do colchete.

Exemplo 2.17 (Algebroide tangente) Se $A$ é um algebroide de Lie sobre uma variedade base $M$ com âncora $\alpha_{A}$ e colchete $[., .]_{A}$, então $T A$ é um algebroide de Lie sobre $T M$ com âncora $\alpha_{T A}=i_{T T M} \circ T \alpha_{A}$, onde $i_{T T M}$ é a involução canônica de $T T M$ que troca as duas projeções $\tau_{T M}$ e $T \tau_{M}$ (veja o diagrama em [1, p. 214]), e colchete $[., .]_{T A}$ definido por

$$
[T \xi, T \eta]_{T A}=T\left([\xi, \eta]_{A}\right),[T \xi, \hat{\eta}]_{T A}=\widehat{[\xi, \eta]},[\hat{\xi}, \hat{\eta}]_{T A}=0 \quad \text { para } \xi, \eta \in \Gamma(A),
$$

onde se usa o fato de que toda seção $\xi$ de $A$ (em relação à projeção original, denotada por $\pi: A \longrightarrow M$, digamos) admite dois levantamentos distintos para uma seção de $T A$ (em relação à projeção tangente da original, $T \pi: T A \longrightarrow T M$ ), a saber: (a) um levantamento $T \xi: T M \longrightarrow T A$ recobrindo a seção original $\xi$, que é simplesmente sua tangente, e (b) um levantamento $\hat{\xi}: T M \longrightarrow T A$ recobrindo a seção zero de $A$, que pode ser explicitamente definida usando a decomposição canônica do fibrado tangente $T A$ de $A$ em cima da seção zero em parte horizontal e parte vertical,

$$
\hat{\xi}\left(v_{x}\right)=T_{x} 0\left(v_{x}\right)+\left.\frac{d}{d t}(t \xi(m))\right|_{t=0},
$$

sendo que em conjunto, estes dois tipos geram o $C^{\infty}(T M)$-módulo de todas as seções de $T A$. Veja, por exemplo, [18].

Para o próximo exemplo, notamos primeiro que, dada uma variedade base $M$, o fibrado de jatos de um fibrado sobre $M$ "herda" algumas das suas possíveis propriedades adicionais. Em particular, o fibrado de jatos de um fibrado vetorial $A$ sobre $M$ é novamente um fibrado vetorial $J A$ sobre $M$ : basta exigir que

$$
j \xi+j \eta=j(\xi+\eta), \lambda j \xi=j(\lambda \xi) \quad \text { para } \lambda \in \mathbb{R}, \xi, \eta \in \Gamma(A) .
$$


Exemplo 2.18 (Algebroide dos jatos) Se $A$ é um algebroide de Lie sobre uma variedade base $M$ com âncora $\alpha_{A}$ e colchete $[., .]_{A}$, então $J A$ é novamente um algebroide de Lie sobre $M$ com âncora $\alpha_{J A}$ e colchete $[., .]_{J A}$ definidas por

$$
\alpha_{J A}(j \xi)=\alpha_{A}(\xi),[j \xi, j \eta]_{J A}=j\left([\xi, \eta]_{A}\right) \quad \text { para } \xi, \eta \in \Gamma(A) .
$$

Aqui é necessário alguma explicação: primeiro, $[., .]_{J A}$ é operador diferencial em ambas as entradas e portanto é local, segundo, localmente $\Gamma(J A)=\langle j \xi\rangle_{C^{\infty}(M)}$ e terceiro, estende por Leibniz para seções arbitrárias.

Notamos que se $A$ é o algebroide de Lie associado a um grupoide de Lie $G$, então a menos de isomorfismo, $J A$ é o algebroide de Lie associado ao grupoide de Lie $J G$ :

$$
J(A G) \cong A(J G) .
$$

Essa construção fica particularmente nítida quando expressa em termos de funções de estrutura. Para isso, escolhamos coordenadas locais $x^{\mu}$ para $M$ e uma base de seções locais $T_{a}$ de $A$, notando que essa induz uma base de seções locais $\left(T_{a}, T_{a}^{\mu}\right)$ de $J A$ de tal modo que se $\xi$ é uma seção de $A$ localmente representada na forma

$$
\xi=\xi^{a} T_{a},
$$

então $j \xi$ é a seção de $J A$ localmente representada na forma

$$
j \xi=\xi^{a} T_{a}+\partial_{\mu} \xi^{a} T_{a}^{\mu} .
$$

Nessa notação, supondo que a âncora e o colchete de $A$ sejam dados por

$$
\alpha_{A}\left(T_{a}\right)=f_{a}^{\mu} \partial_{\mu}
$$

$\mathrm{e}$

$$
\left[T_{a}, T_{b}\right]_{A}=f_{a b}^{c} T_{c}
$$

conforme a equação (2.79), então a âncora e o colchete de $J A$ são dados por

$$
\begin{gathered}
\alpha_{J A}\left(T_{a}\right)=f_{a}^{\mu} \partial_{\mu} \\
\alpha_{J A}\left(T_{a}^{\mu}\right)=0
\end{gathered}
$$

$\mathrm{e}$

$$
\begin{gathered}
{\left[T_{a}, T_{b}\right]_{J A}=f_{a b}^{c} T_{c}+\partial_{\mu} f_{a b}^{c} T_{c}^{\mu}} \\
{\left[T_{a}, T_{b}^{\mu}\right]_{J A}=f_{a b}^{c} T_{c}^{\mu}+\partial_{\nu} f_{a}^{\mu} T_{b}^{\nu}} \\
{\left[T_{a}^{\mu}, T_{b}^{\nu}\right]_{J A}=f_{a}^{\nu} T_{b}^{\mu}-f_{b}^{\nu} T_{a}^{\mu}}
\end{gathered}
$$

Veja, por exemplo, [3]. 
O exemplo final requer introduzir a noção de uma ação infinitesimal de um algebroide de Lie. ${ }^{9}$ Para tanto, recordamos primeiro que, dado um fibrado $E$ sobre uma variedade base $M$, com projeção $\pi$, um campo vetorial $X_{E}$ sobre $E$ é chamado de projetável se vale

$$
T_{e_{1}} \pi\left(X_{E}\left(e_{1}\right)\right)=T_{e_{2}} \pi\left(X_{E}\left(e_{2}\right)\right) \quad \text { quando } \pi\left(e_{1}\right)=\pi\left(e_{2}\right) .
$$

Uma condição equivalente é que exista um (único) campo vetorial $X_{M}$ sobre $M$ que é $\pi$-relacionado a $X_{E}$, chamado a sua projeção,

$$
T_{e} \pi\left(X_{E}(e)\right)=X_{M}(\pi(e))
$$

Obviamente, campos vetoriais verticais são projetáveis: $X_{E}$ é vertical se e somente se $X_{E}$ é projetável com $X_{M}=0$. Prova-se que, dentro da álgebra de Lie $\mathfrak{X}(E)$ de todos os campos vetoriais sobre $E$, os campos projetáveis e os campos verticais formam subálgebras de Lie que denotaremos por $\mathfrak{X}_{P}(E)$ e por $\mathfrak{X}_{V}(E)$, respectivamente, sendo que a segunda é de fato um ideal na primeira. A importância dessas noções deriva do fato de que, se considerarmos $\mathfrak{X}(E)$ como sendo, formalmente, a álgebra de Lie do grupo $\operatorname{Diff}(E)$ dos difeomorfismos de $E$, então $\mathfrak{X}_{P}(E)$ é a álgebra de Lie do grupo $\operatorname{Aut}(E)$ dos automorfismos de $E$ e $\mathfrak{X}_{V}(E)$ é a álgebra de Lie do grupo $\operatorname{Aut}_{s}(E)$ dos automorfismos estritos de $E$ : o fluxo de um campo projetável consiste de automorfismos locais e o fluxo de um campo vertical consiste de automorfismos locais estritos.

Definição 2.8 Seja A um algebroide de Lie sobre uma variedade base $M$, com âncora $\alpha$ e colchete [.,.], e seja $E$ um fibrado sobre a mesma variedade base $M$, com projeção $\pi$. Uma ação infinitesimal de A sobre E é um homomorfismo de álgebras de Lie

$$
\begin{aligned}
\dot{\Phi}_{E}: \Gamma(A) & \longrightarrow \mathfrak{X}_{P}(E) \\
X & \longmapsto X_{E}
\end{aligned}
$$

que é compativel com as estruturas de módulo sobre os anéis de funçôes pertinentes,

$$
(f X)_{E}=(f \circ \pi) X_{E} \quad \text { para } f \in C^{\infty}(M), X \in \Gamma(A),
$$

e tal que a âncora corresponde à projeção,

$$
X_{M}=\alpha(X) \quad \text { para } X \in \Gamma(A) .
$$

Continuamos chamando $X_{E}$ o campo fundamental associado a $X$.

\footnotetext{
${ }^{9} \mathrm{Na}$ literatura, muitos autores omitem o adjetivo "infinitesimal", sendo que seguiremos este abuso de linguagem conforme conveniência.
} 
A terminologia se justifica quando observamos que, dado um fibrado $E$ sobre uma variedade base $M$, com projeção $\pi$, e um grupoide de Lie $G$ sobre a mesma variedade base $M$, com projeção fonte $\sigma$ e projeção alvo $\tau$, assim como seu algebroide de Lie $A G$, com âncora $\alpha$ e colchete [.,.], então uma ação de $G$ sobre $E$ induz uma ação infinitesimal de $A G$ sobre $E$, definida pela fórmula usual: lembrando que, para $X \in \Gamma(A G)$ e $x \in M$,

$$
X(x)=\left.\frac{d}{d t} \exp (t X)(x)\right|_{t=0},
$$

temos, para $X \in \Gamma(A G)$ e $e \in E \operatorname{com} x=\pi(e) \in M$,

$$
X_{E}(e)=\left.\frac{d}{d t} \exp (t X)(x) \cdot e\right|_{t=0} .
$$

Exemplo 2.19 (Algebroide de ação) Se $E$ é um fibrado sobre uma variedade base $M$ com projeção $\pi$, munido de uma ação infinitesimal de um algebroide de Lie $A$ sobre a mesma variedade base $M$, com âncora $\alpha_{A}$ e colchete $[., .]_{A}$, o produto fibrado $A \times_{M} E$ pode ser munido da estrutura de um algebroide de Lie sobre o espaço total $E$ do fibrado original, que a seguir será denotado por $A \varangle E$, com âncora $\alpha_{A \varangle E}$ e colchete $[., .]_{A \varangle E}$ definidas por

$$
\alpha_{G \varangle E}\left(\pi^{*} X\right)=X_{E} \quad \text { para } X \in \Gamma(A)
$$

e

$$
\left[\pi^{*} X, \pi^{*} Y\right]_{G \varangle E}=\pi^{*}\left([X, Y]_{A}\right) \quad \text { para } X, Y \in \Gamma(A)
$$

onde notamos que, como fibrado vetorial sobre $E, A \varangle E$ é simplesmente o "pull-back" $\pi^{*} A$ de $A$ pela projeção $\pi$ e que o "pull-back" com $\pi$ de seções de $A$ proporciona seções de $A \varangle E$ que são "constantes ao longo das fibras" de $E$, sendo que o espaço de todas as seções de $A \varangle E$ pode ser obtido formando combinações lineares destas com coeficientes que são funções quaisquer sobre $E$; então as propriedades exigidas de uma ação infinitesimal garantem que as equações anteriores determinam uma âncora bem definida e um colchete bem definido que satisfazem as propriedades exigidas de um algebroide de Lie.

Notamos que se $A$ é o algebroide de Lie associado a um grupoide de Lie $G$, então a menos de isomorfismo, o algebroide de ação $A \varangle E$ é o algebroide de Lie associado ao grupoide de ação $G \varangle E$ :

$$
A G \varangle E \cong A(G \varangle E) .
$$

Concluímos com uma breve discussão da "versão infinitesimal" das construções envolvendo bisseções do grupoide de ação apresentados no final da subseção anterior. Primeiro, o "pull-back" de seções com a projeção $\pi$ usado no exemplo acima proporciona um homomorfismo de álgebras de Lie

$$
\begin{array}{ccc}
\Gamma(A) & \longrightarrow \Gamma(A \varangle E) \\
X & \longmapsto & \pi^{*} X
\end{array}
$$


que podemos considerar a "versão infinitesimal" do homomorfismo de grupos (2.71), definido por

$$
\pi^{*} X=(X \circ \pi, 0),
$$

que é a "versão infinitesimal" de (2.72). Segundo, a âncora do algebroide de ação é um homomorfismo de álgebras de Lie

$$
\begin{array}{clc}
\Gamma(A \varangle E) & \longrightarrow \mathfrak{X}(E) \\
Z & \longmapsto \alpha_{G \varangle E}(Z)
\end{array}
$$

que podemos considerar a "versão infinitesimal" do homomorfismo de grupos (2.73). Compondo, recuperamos a ação infinitesimal original, como homomorfismo de álgebras de Lie

$$
\begin{gathered}
\Gamma(A) \quad \longrightarrow \mathfrak{X}_{P}(E) \\
X \quad \longmapsto X_{E}
\end{gathered}
$$

que podemos considerar a "versão infinitesimal" do homomorfismo de grupos (2.74). Finalmente, nota-se que o diagrama

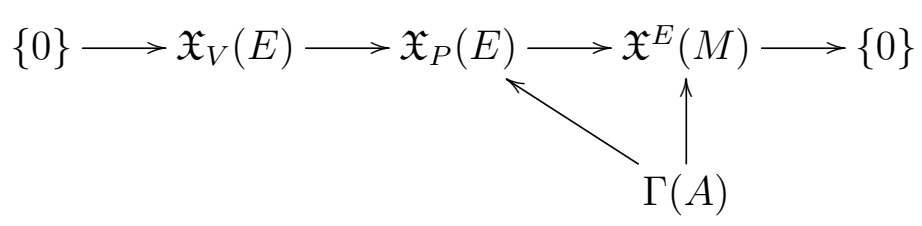

comuta, onde $\mathfrak{X}^{E}(M)$ denota a álgebra dos campos vetoriais sobre $M$ que admitem algum levantamento para um campo vetorial projetável sobre $E$. 



\section{Capítulo 3}

\section{Resultados}

É neste capítulo que apresentamos o resultado principal da tese, que é a generalização do teorema de Noether ao caso em que a simetria do sistema em questão é realizada pela ação de um grupoide de Lie, e não de um grupo de Lie, generalização essa que enunciamos e demonstramos na Seção 3.3 e que na literatura existente não está sendo contemplada em toda sua plenitude. De fato, mesmo no âmbito de uma abordagem geométrica à teoria clássica dos campos tal como a geometria multissimplética [10], simetrias costumam ser descritas por grupos de automorfismos dos fibrados subjacentes: estes grupos podem ser grupos de Lie de dimensão finita (para descrever simetrias globais) ou grupos "menos bem comportados" de dimensão infinita (para descrever simetrias locais), sendo que os automorfismos empregados podem atuar trivialmente no espaço-tempo (no caso de simetrias internas ou de calibre) ou não (no caso de que nós chamaremos de simetrias externas ou de espaço-tempo). Um primeiro passo na direção de "localizar" estes automorfismos em relação à sua dependência espaço-temporal foi dado em [8], onde essa ideia foi elaborada para o caso de simetrias internas, ou simetrias de calibre, as quais não movem os pontos do espaço-tempo: neste caso, os grupos tradicionais mencionados acima são subgrupos do grupo $\operatorname{Aut}_{s}(P)$ dos automorfismos estritos do fibrado principal $P$ da teoria, que também pode ser realizado como o grupo das seções de um certo fibrado de grupos de Lie sobre o espaço-tempo, a saber, o fibrado associado $P \times_{G} G$. O que falta ainda é estender essa abordagem a simetrias externas, onde os grupos tradicionais mencionados acima se tornam subgrupos do grupo $\operatorname{Aut}(P)$ dos automorfismos do fibrado principal $P$ da teoria, que por sua vez pode ser realizado como o grupo das bisseções de um certo grupoide de Lie sobre o espaço-tempo, a saber, o grupoide de calibre $(P \times P) / G$.

A dificuldade principal a ser superada na implementação deste programa provém de um fenômeno que não possui análogo na abordagem tradicional: refere-se à noção de "ação induzida". Dados um grupo de Lie $G$ e uma ação de $G$ em uma variedade $E$, obtemos por 
diferenciação ações induzidas do mesmo grupo de Lie $G$ em variedades que derivam de $E$, tais como seu fibrado tangente $T E$ e seus descendentes (o fibrado cotangente $T^{*} E$ e os demais fibrados tensoriais $T_{s}^{r} E$ ); sendo assim, torna-se óbvio o significado da afirmação de que um campo tensorial sobre $E$ (em particular, uma forma diferencial sobre $E$ ) seja invariante sob a ação de $G$. Mas dados um grupoide de Lie $G$ sobre uma variedade base $M$ e uma ação de $G$ em um fibrado $E$ sobre a mesma variedade base $M$, obtemos por diferenciação ações induzidas de outros grupoides de Lie em fibrados que derivam de $E$. Por exemplo, passando do grupoide original $G$ para o seu grupoide dos jatos $J G$, podemos obter uma ação induzida de $J G$ sobre o fibrado dos jatos $J E$ de $E$ e os seus descendentes, mas como não existe nenhum mergulho natural de $G$ em $J G$ como subgrupoide de Lie, tal "prolongamento" da ação original não pode ser interpretado como uma extensão. De modo semelhante, passando do grupoide original $G$ para o seu grupoide tangente $T G$, podemos obter uma ação induzida de $T G$ sobre o fibrado tangente $T E$ de $E$ e os seus descendentes, mas aqui o maior obstáculo reside no fato de que, para tanto, é preciso mudar de variedade base: da variedade original $M$ para seu fibrado tangente $T M$. Tal mudança de base acaba tornando essa construção inútil para aplicações em teoria dos campos, pois campos são seções de fibrados sobre o espaço-tempo e não sobre outros espaços que são maiores.

A saída deste impasse é proporcionada pela construção de uma ação induzida do grupoide dos jatos $J G$ de $G$ sobre o fibrado tangente $T E$ de $E$ e os seus descendentes que, pelo conhecimento do autor, é nova. Ela permite definir, sem qualquer grau de ambiguidade, quando um campo tensorial sobre o espaço total $E$ é invariante em relação à ação de $J G$ (não de $G$ ) ou de um subgrupoide de $J G$.

Um outro conceito, também recente mas já conhecido e que se mostra importante na solução do problema abordado nesta tese, é o de um grupoide de Pfaff [19]: é um grupoide de Lie $G$ munido de um certo tipo de distribuição que define um subgrupoide de Lie do grupoide tangente $T G$ de $G$. Para mais detalhes veja Apêndice A.

Combinando técnicas sugeridas por estas construções, conseguimos elucidar em que sentido - ou mais precisamente, em relação a qual ação induzida de qual grupoide - a forma multissimplética $\omega$ do formalismo covariante hamiltoniano (assim como a forma multicanônica $\theta$ da qual ela é a derivada exterior) é invariante, o que significa invariância da hamiltoniana de um modelo e, por fim, como formular o teorema de Noether neste âmbito.

Colocado o problema, podemos esboçar o plano de trabalho para o presente capítulo. A primeira seção se dedica à discussão de ações induzidas de vários tipos que aparecem em seguida. Na segunda seção, discutimos a invariância das formas $\theta$ e $\omega$, de uma hamiltoniana $\mathcal{H}$ e das formas $\theta_{\mathcal{H}}$ e $\omega_{\mathcal{H}}$ que ela induz por "pull-back". Assim, na terceira seção, 
estamos finalmente em condições de enunciar e provar o resultado principal desta tese: o teorema de Noether no âmbito de grupoides.

\subsection{Ações induzidas}

Durante toda esta seção, $E$ será um fibrado sobre uma variedade base $M \operatorname{com}$ projeção $\pi_{E}$, munido de uma ação

$$
\begin{aligned}
\Phi_{E}: G \times_{M} E & \longrightarrow E \\
(g, e) & \longmapsto g \cdot e
\end{aligned}
$$

de um grupoide de Lie $G$ sobre a mesma variedade base $M$, com projeção fonte $\sigma_{G}$ e projeção alvo $\tau_{G}$, multiplicação $\mu_{G}$, unidade $1_{G}$ e inversão $\iota_{G}$, como no Exemplo 2.7.

\subsubsection{Fibrado vertical}

O primeiro e mais elementar exemplo de ação induzida, no qual o grupoide que age não é modificado, é obtido considerando o fibrado vertical $V E$ de $E$ : por diferenciação de curvas verticais, obtemos uma ação

$$
\begin{aligned}
\Phi_{V E}: G \times_{M} V E & \longrightarrow V E \\
\left(g, v_{e}\right) & \longmapsto g \cdot v_{e}
\end{aligned}
$$

do grupoide $G$ sobre o fibrado vertical $V E$ de $E$, explicitamente definida da seguinte maneira: dados $g \in G$ com $\sigma_{G}(g)=x, e \in E$ com $\pi_{E}(e)=x$ e uma curva $t \mapsto e(t)$ em $E$ que passa pelo ponto $e$ (i.e., $e(0)=e$ ) e é vertical (i.e., $\pi_{E}(e(t))=x=\sigma_{G}(g)$ independe de $t$ ), então

$$
\left.g \cdot \frac{d}{d t} e(t)\right|_{t=0}=\left.\frac{d}{d t} g \cdot e(t)\right|_{t=0} .
$$

(O fato de $\Phi_{V E}$ ser uma ação segue diretamente da hipótese de $\Phi_{E}$ ser uma ação, aplicando a regra da cadeia.)

Uma propriedade importante da ação (3.2) é que ela respeita a estrutura de $V E$ como fibrado vetorial sobre $E$, pois (a) ela recobre a ação original, ou seja, o diagrama

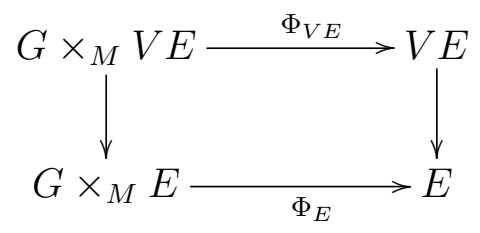


comuta, e (b) para qualquer $g \in G$, a translação à esquerda por $g$ é bem definida sobre o espaço vetorial $V_{e} E$ inteiro se valer $\pi_{E}(e)=\sigma_{G}(g)$ (enquanto que, caso contrário, não é bem definida em nenhum dos seus pontos), e sua restrição a este espaço é uma transformação linear $L_{g}: V_{e} E \longrightarrow V_{g \cdot e} E$, pois é a aplicação tangente, em $e$, à translação à esquerda $L_{g}: E_{x} \longrightarrow E_{y}$, onde $x=\sigma_{G}(g)=\pi_{E}(e), y=\tau_{G}(g)=\pi_{E}(g \cdot e)$.

\subsubsection{Fibrados dos jatos e descendentes}

Por outro lado, aplicando o "funtor $J "$ a todas as aplicações estruturais do parágrafo inicial desta seção, obtemos uma ação

$$
\begin{aligned}
\Phi_{J E}: J G \times_{M} J E & \longrightarrow J E \\
\left(u_{g}, u_{e}\right) & \longmapsto u_{g} \cdot u_{e}
\end{aligned}
$$

do grupoide dos jatos $J G$ de $G$ sobre o fibrado dos jatos $J E$ de $E$, explicitamente definida da seguinte maneira: dados $g \in G \operatorname{com} \sigma_{G}(g)=x$ e $\tau_{G}(g)=y, e \in E \operatorname{com} \pi_{E}(e)=x$, $u_{g} \in J_{g} G \subset L\left(T_{x} M, T_{g} G\right)$ e $u_{e} \in J_{e} E \subset L\left(T_{x} M, T_{e} E\right)$, concatenamos os dois em uma aplicação linear $\left(u_{g}, u_{e}\right) \in L\left(T_{x} M, T_{g} G \oplus T_{e} E\right.$ ) (que na verdade toma valores em $L\left(T_{x} M, T_{(g, e)}\left(G \times_{M} E\right)\right)$ pois $\left.T_{x} \sigma_{G} \circ u_{g}=\operatorname{id}_{T_{x} M}=T_{e} \pi_{E} \circ u_{e}\right)$ e compomos:

$$
u_{g} \cdot u_{e}=T_{(g, e)} \Phi_{E} \circ\left(u_{g}, u_{e}\right) \circ\left(T_{g} \tau \circ u_{g}\right)^{-1} .
$$

(O fato de $\Phi_{J E}$ ser uma ação segue diretamente da hipótese de $\Phi_{E}$ ser uma ação, aplicando a regra da cadeia.) Pode-se provar então que o grupoide de ação induzido por esta ação é canonicamente isomorfo ao grupoide dos jatos do grupoide de ação induzido pela ação original (ambos são grupoides de Lie sobre $J E$ ):

$$
J G \varangle J E \cong J(G \varangle E) .
$$

Um procedimento muito semelhante também proporciona uma ação

$$
\begin{aligned}
\Phi_{\vec{J} E}: \quad J G \times_{M} \vec{J} E & \longrightarrow \overrightarrow{J E} \\
\left(u_{g}, \vec{u}_{e}\right) & \longmapsto u_{g} \cdot \vec{u}_{e}
\end{aligned}
$$

do grupoide dos jatos $J G$ de $G$ sobre o fibrado dos jatos linearizados $\overrightarrow{J E}$ de $E$ que, ao contrário da anterior, admite uma simplificação, pois fatoriza na composição do morfismo (2.69) de grupoides de Lie com uma ação

$$
\begin{array}{ccc}
\left(G L(T M) \times_{M} G\right) \times_{M} \overrightarrow{J E} & \longrightarrow & \vec{J} E \\
\left((a, g), \vec{u}_{e}\right) & \longmapsto & (a, g) \cdot \vec{u}_{e}
\end{array}
$$


de $G L(T M) \times{ }_{M} G$ sobre $\overrightarrow{J E}$, na qual o grupoide de Lie pertinente é o produto fibrado do grupoide $G L(T M)$ dos referenciais lineares de $M$ com o grupoide de Lie original $G$. Essa ação é sugerida pelo isomorfismo (2.7), junto ao fato de que o fibrado tangente e o fibrado cotangente de $M$ vêem munidos de uma a ação natural do grupoide $G L(T M)$ dos referenciais lineares de $M$ e o fibrado vertical de $E$ vem munido de uma ação induzida de $G$ conforme explicado acima, mas temos que levar em conta que todos os grupoides envolvidos são grupoides sobre $M$ enquanto que o isomorfismo (2.7) é um de fibrados vetoriais sobre $E$. Assim, vale especificar que a ação (3.9) é explicitamente definida da seguinte maneira: dados $(a, g) \in G L(T M) \times_{M} G \operatorname{com} \sigma_{G L(T M)}(a)=x=\sigma_{G}(g)$ e $\tau_{G L(T M)}(a)=y=\tau_{G}(g), e \in E$ com $\pi_{E}(e)=x$ e $\vec{u}_{e} \in \vec{J}_{e} E=L\left(T_{x} M, V_{e} E\right)$, obtemos $(a, g) \cdot \vec{u}_{e} \in \vec{J}_{g \cdot e} E=L\left(T_{y} M, V_{g \cdot e} E\right)$ por composição:

$$
(a, g) \cdot \vec{u}_{e}=L_{g} \circ \vec{u}_{e} \circ a^{-1}
$$

onde $L_{g}$ é a translação à esquerda por $g$ em $V E$ introduzida no final da subseção anterior.

Uma propriedade importante das ações (3.5) e (3.8) é que elas respeitam a estrutura de $J E$ como fibrado afim e de $\overrightarrow{J E}$ como fibrado vetorial sobre $E$, pois (a) elas recobrem a ação original, ou seja, os diagramas

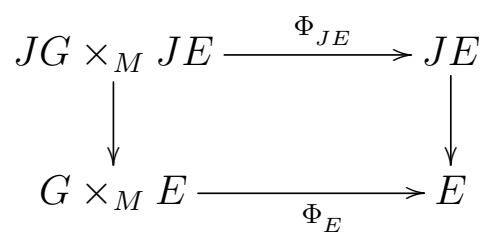

$\mathrm{e}$

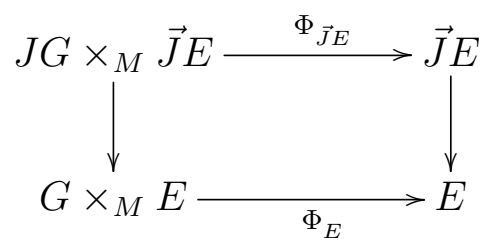

comutam, e (b) para qualquer $g \in G$ e qualquer $u_{g} \in J_{g} G$, a translação à esquerda por $u_{g}$ é bem definida sobre o espaço afim $J_{e} E$ inteiro e sobre o espaço vetorial $\overrightarrow{J_{e}} E$ inteiro se valer $\pi_{E}(e)=\sigma_{G}(g)$ (enquanto que, caso contrário, não é bem definida em nenhum dos seus pontos), e sua restrição a este espaço é uma transformação afim $L_{u_{g}}: J_{e} E \longrightarrow J_{g \cdot e} E$ ou transformação linear $L_{u_{g}}: \vec{J}_{e} E \longrightarrow \vec{J}_{g \cdot e} E$.

Essa afirmação é óbvia para o caso dos jatos linearizados, a partir da equação (3.10), de modo que só precisamos verificá-la para o caso dos jatos. Fixamos $x \in M, g \in G$ e $e \in E \operatorname{com} \sigma_{G}(g)=x=\pi_{E}(e)$ e ainda $u_{g} \in J_{g} G$ : 
então conforme a definição (3.6), a translação à esquerda $L_{u_{g}}: J_{e} E \longrightarrow J_{g \cdot e} E$ pode ser obtida como composição das seguintes aplicações: primeiro, a aplicação

$$
\begin{array}{ccc}
J_{e} E & \longrightarrow & L\left(T_{x} M, T_{(g, e)}\left(G \times_{M} E\right)\right) \\
u_{e} & \longmapsto & \left(u_{g}, u_{e}\right)
\end{array}
$$

que, sendo a restrição da aplicação afim ${ }^{1}$

$$
\begin{array}{ccc}
L\left(T_{x} M, T_{e} E\right) & \longrightarrow L\left(T_{x} M, T_{g} G \oplus T_{e} E\right) \cong L\left(T_{x} M, T_{g} G\right) \oplus L\left(T_{x} M, T_{e} E\right) \\
u_{e} & \longmapsto & \left(u_{g}, u_{e}\right)
\end{array}
$$

é afim, e segundo, a aplicação linear

$$
\begin{array}{clc}
L\left(T_{x} M, T_{(g, e)}\left(G \times_{M} E\right)\right) & L\left(T_{y} M, T_{g \cdot e} E\right) \\
w & \longmapsto T_{(g, e)} \Phi_{E} \circ w \circ\left(T_{g} \tau \circ u_{g}\right)^{-1}
\end{array}
$$

Por fim, comparando as equações (3.6) e (3.10), observa-se que, por construção, a "aplicação diferença"

$$
J E \times_{E} J E \longrightarrow \overrightarrow{J E}
$$

que a cada dois jatos $u_{1}, u_{2} \in J_{e} E \subset L\left(T_{x} M, T_{e} E\right)$ no mesmo ponto $e$ de $E$ associa o jato linearizado que é a sua diferença $u_{1}-u_{2} \in \vec{J}_{e} E=L\left(T_{x} M, V_{e} E\right)$, é $J G$-equivariante.

A compatibilidade das ações (3.5) e (3.8) com a estrutura de $J E$ como fibrado afim e de $\vec{J} E$ como fibrado vetorial sobre $E$ permite transferir essas ações de jatos para cojatos (torcidos), por dualização. Assim, obtemos uma ação

$$
\begin{aligned}
\Phi_{J \circledast E}: \quad J G \times_{M} J^{\circledast} E & \longrightarrow J^{\circledast} E \\
\left(u_{g}, z_{e}\right) & \longmapsto u_{g} \cdot z_{e}
\end{aligned}
$$

de $J G$ sobre $J^{\circledast} E$ definida do seguinte modo: para $g \in G \operatorname{com} \sigma_{G}(g)=x$ e $\tau_{G}(g)=y$, $e \in E \operatorname{com} \pi_{E}(e)=x, u_{g} \in J_{g} G, z_{e} \in J_{e}^{\circledast} E$ e $u_{g \cdot e} \in J_{g \cdot e} E$,

$$
\left\langle u_{g} \cdot z_{e}, u_{g \cdot e}\right\rangle=\left(T_{g} \tau \circ u_{g}\right)^{-1^{*}}\left\langle z_{e}, u_{g}^{-1} \cdot u_{g \cdot e}\right\rangle,
$$

prescrição que pode ser melhor compreendida pelo seguinte diagrama:

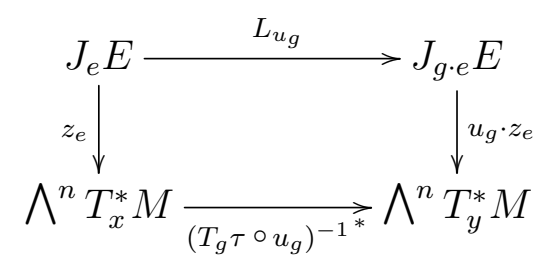

\footnotetext{
${ }^{1}$ Aqui, usamos que para quaisquer dois espaços vetoriais $E$ e $F$ e qualquer vetor $u \in E$, a aplicação de $E$ em $E \oplus F$ que leva $v$ em $(u, v)$ é afim, tendo como aplicação linear associada a inclusão de $E$ em $E \oplus F$ que leva $v$ em $(0, v)$.
} 
De modo semelhante, obtemos uma ação

$$
\begin{aligned}
\Phi_{\vec{J}^{\circledast E}}: \quad J G \times_{M} \vec{J}^{\circledast} E & \longrightarrow \vec{J}^{\circledast} E \\
\left(u_{g}, \vec{z}_{e}\right) & \longmapsto u_{g} \cdot \vec{z}_{e}
\end{aligned}
$$

de $J G$ sobre $\vec{J}{ }^{\circledast} E$ que fatoriza na composição do morfismo (2.69) de grupoides de Lie com uma ação

$$
\begin{array}{ccc}
\left(G L(T M) \times_{M} G\right) \times_{M} \vec{J}^{\circledast} E & \longrightarrow \quad \vec{J}^{\circledast} E \\
\left((a, g), \vec{z}_{e}\right) & \longmapsto & (a, g) \cdot \vec{z}_{e}
\end{array}
$$

de $G L(T M) \times_{M} G$ sobre $\vec{J}{ }^{\circledast} E$ definida do seguinte modo: para $(a, g) \in G L(T M) \times_{M} G$ $\operatorname{com} \sigma_{G L(T M)}(a)=x=\sigma_{G}(g)$ e $\tau_{G L(T M)}(a)=y=\tau_{G}(g), e \in E \operatorname{com} \pi_{E}(e)=x$, $\vec{z}_{e} \in \vec{J}_{e}^{\circledast} E$ e $\vec{u}_{g \cdot e} \in \vec{J}_{g \cdot e} E$

$$
\left\langle(a, g) \cdot \vec{z}_{e}, \vec{u}_{g \cdot e}\right\rangle=a^{-1^{*}}\left\langle\vec{z}_{e},(a, g)^{-1} \cdot \vec{u}_{g \cdot e}\right\rangle .
$$

prescrição que pode ser melhor compreendida pelo seguinte diagrama:

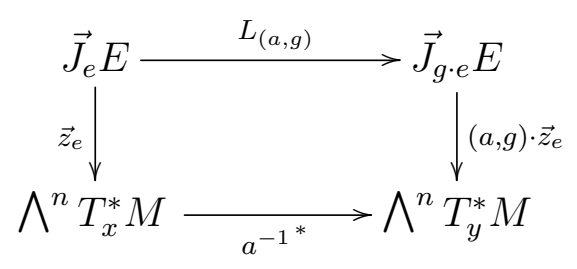

Todas essas ações desfrutam das mesmas propriedades de compatibilidade com as respectivas estruturas adicionais de fibrado vetorial sobre $E$ que antes.

\subsubsection{Fibrado tangente (parte 1)}

Agora, aplicando o "funtor $T$ " a todas as aplicações estruturais do parágrafo inicial desta seção, obtemos uma ação

$$
\begin{array}{ccc}
T G \times_{T M} T E & \longrightarrow T E \\
\left(v_{g}, v_{e}\right) & \longmapsto & v_{g} \cdot v_{e}
\end{array}
$$

do grupoide tangente $T G$ de $G$ sobre o fibrado tangente $T E$ de $E$, definida pela aplicação tangente $T \Phi_{E}$ à aplicação $\Phi_{E}$, mediante a identificação canônica de $T G \times_{T M} T E$ com $T\left(G \times \times_{M} E\right)$, a qual expressa o fato de que um par de vetores $\left(v_{g}, v_{e}\right) \in T_{g} G \oplus T_{e} E$ pertence ao subespaço $\left(T G \times_{T M} T E\right)_{(g, e)}=T_{(g, e)}\left(G \times_{M} E\right)$ se e somente se valer

$$
T_{g} \sigma_{G}\left(v_{g}\right)=T_{e} \pi_{E}\left(v_{e}\right)
$$


Pode-se provar então que o grupoide de ação induzido por esta ação é canonicamente isomorfo ao grupoide tangente do grupoide de ação induzido pela ação original (ambos são grupoides de Lie sobre $T E$ ):

$$
T G \varangle T E \cong T(G \varangle E) .
$$

Infelizmente, porém, a ação (3.21), apesar de recobrir a ação original no sentido de que o diagrama

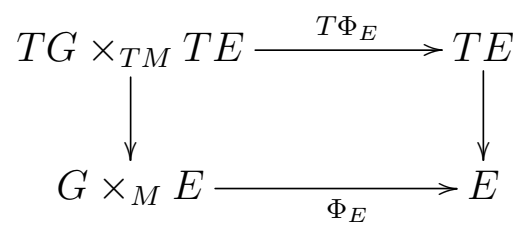

comuta, não respeita a estrutura de $T E$ como fibrado vetorial sobre $E$. De fato, para qualquer $g \in G$ e qualquer $v_{g} \in T_{g} G$, a translação à esquerda por $v_{g}$ não é bem definida sobre o espaço vetorial $T_{e} E$ inteiro, mesmo se valer $\pi_{E}(e)=\sigma_{G}(g)$, mas apenas sobre seu subespaço afim $\left(T_{e} \pi_{E}\right)^{-1}\left(T_{g} \sigma_{G}\left(v_{g}\right)\right)$, sendo que sua restrição a este subespaço é uma transformação afim $L_{v_{g}}:\left(T_{e} \pi_{E}\right)^{-1}\left(T_{g} \sigma_{G}\left(v_{g}\right)\right) \longrightarrow\left(T_{g \cdot e} \pi_{E}\right)^{-1}\left(T_{g} \tau_{G}\left(v_{g}\right)\right)$. Tal falha impede a transferência desta ação de $T G$ de vetores tangentes para vetores cotangentes ou tensores em geral, o que acaba deixando-a essencialmente inútil. Como veremos logo em seguida, a saída deste impasse consiste em mudar o grupoide, de $T G$ para $J G$.

\subsubsection{Fibrado tangente (parte 2) e descendentes}

Tendo em vista a dificuldade encontrada na subseção anterior, passamos agora a construir uma ação

$$
\begin{aligned}
\Phi_{T E}: J G \times_{M} T E & \longrightarrow T E \\
\left(u_{g}, v_{e}\right) & \longmapsto u_{g} \cdot v_{e}
\end{aligned}
$$

do grupoide dos jatos $J G$ de $G$ sobre o fibrado tangente $T E$ de $E$ que ainda é definida usando a aplicação tangente $T \Phi_{E}$ à ação $\Phi_{E}$ original, porém de outra forma: para $g \in G$ e $e \in E \operatorname{com} \sigma_{G}(g)=\pi_{E}(e), u_{g} \in J_{g} G$ e $v_{e} \in T_{e} E$, define-se

$$
u_{g} \cdot v_{e}=T_{(g, e)} \Phi_{E}\left(\left(u_{g} \circ T_{e} \pi_{E}\right)\left(v_{e}\right), v_{e}\right)
$$

Notando que

$$
\begin{aligned}
T_{g \cdot e} \pi_{E}\left(u_{g} \cdot v_{e}\right) & =T_{(g, e)}\left(\pi_{E} \circ \Phi_{E}\right)\left(\left(u_{g} \circ T_{e} \pi_{E}\right)\left(v_{e}\right), v_{e}\right) \\
& =T_{(g, e)}\left(\tau_{G} \circ \operatorname{pr}_{1}\right)\left(\left(u_{g} \circ T_{e} \pi_{E}\right)\left(v_{e}\right), v_{e}\right) \\
& =T_{g} \tau_{G}\left(\left(u_{g} \circ T_{e} \pi_{E}\right)\left(v_{e}\right)\right)=\left(T_{g} \tau_{G} \circ u_{g}\right)\left(T_{e} \pi_{E}\left(v_{e}\right)\right)
\end{aligned}
$$


obtemos que, para $g, h \in G$ e $e \in E \operatorname{com} \sigma_{G}(g)=\pi_{E}(e), \sigma_{G}(h)=\tau_{G}(g)=\pi_{E}(g \cdot e)$ e $u_{g} \in J_{g} G, u_{h} \in J_{h} G, v_{e} \in T_{e} E$, vale

$$
\begin{aligned}
u_{h} \cdot\left(u_{g} \cdot v_{e}\right) & =T_{(h, g \cdot e)} \Phi_{E}\left(\left(u_{h} \circ T_{g \cdot e} \pi_{E}\right)\left(u_{g} \cdot v_{e}\right), u_{g} \cdot v_{e}\right) \\
& =T_{(h, g \cdot e)} \Phi_{E}\left(\left(u_{h} \circ\left(T_{g} \tau_{G} \circ u_{g}\right) \circ T_{e} \pi_{E}\right)\left(v_{e}\right), T_{(g, e)} \Phi_{E}\left(\left(u_{g} \circ T_{e} \pi_{E}\right)\left(v_{e}\right), v_{e}\right)\right. \\
& =T_{(h, g, e)}\left(\Phi_{E} \circ\left(\operatorname{id}_{G} \times \Phi_{E}\right)\right)\left(\left(u_{h} \circ\left(T_{g} \tau_{G} \circ u_{g}\right) \circ T_{e} \pi_{E}\right)\left(v_{e}\right),\left(u_{g} \circ T_{e} \pi_{E}\right)\left(v_{e}\right), v_{e}\right) \\
& =T_{(h, g, e)}\left(\Phi_{E} \circ\left(\mu_{G} \times \mathrm{id}_{E}\right)\right)\left(\left(u_{h} \circ\left(T_{g} \tau_{G} \circ u_{g}\right) \circ T_{e} \pi_{E}\right)\left(v_{e}\right),\left(u_{g} \circ T_{e} \pi_{E}\right)\left(v_{e}\right), v_{e}\right) \\
& =T_{(h g, e)} \Phi_{E}\left(\left(T_{(h, g)} \mu_{G} \circ\left(u_{h} \circ\left(T_{g} \tau_{G} \circ u_{g}\right), u_{g}\right) \circ T_{e} \pi_{E}\right)\left(v_{e}\right), v_{e}\right) \\
& =T_{(h g, e)} \Phi_{E}\left(\left(u_{h} u_{g} \circ T_{e} \pi_{E}\right)\left(v_{e}\right), v_{e}\right) \\
& =\left(u_{h} u_{g}\right) \cdot v_{e}
\end{aligned}
$$

$\mathrm{e}, \operatorname{com} \sigma_{G}(g)=x=\pi_{E}(e)$,

$$
\begin{aligned}
1_{J G, x} \cdot v_{e} & =T_{1_{G}, x} \Phi_{E}\left(\left(T_{x} 1_{G} \circ T_{e} \pi_{E}\right)\left(v_{e}\right), v_{e}\right) \\
& =T_{e}\left(\Phi_{E} \circ\left(1_{G} \circ \pi_{E}, \operatorname{id}_{E}\right)\right)\left(v_{e}\right) \\
& =T_{e} \operatorname{id}_{E}\left(v_{e}\right)=v_{e}
\end{aligned}
$$

provando assim que a aplicação (3.24) definida pela equação (3.25) é de fato uma ação. Notamos também que a equação (3.26) acima mostra que essa ação preserva o fibrado vertical $V E$ de $E$, de modo que por restrição, a ação (3.24) induz uma ação

$$
\begin{aligned}
\Phi_{V E}: J G \times_{M} V E & \longrightarrow V E \\
\left(u_{g}, v_{e}\right) & \longmapsto u_{g} \cdot v_{e}
\end{aligned}
$$

do grupoide dos jatos $J G$ de $G$ sobre o fibrado vertical $V E$ de $E$, a qual é idêntica à composição da ação (3.2) com o morfismo de grupoides de Lie do Exemplo 2.10.

Mais uma vez, uma propriedade importante da ação (3.24) é que, esta sim, respeita a estrutura de $T E$ como fibrado vetorial sobre $E$, pois (a) ela recobre a ação original, ou seja, o diagrama

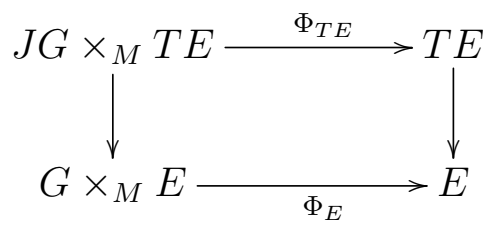

comuta, e (b) para qualquer $g \in G$ e qualquer $u_{g} \in J_{g} G$, a translação à esquerda por $u_{g}$ é bem definida sobre o espaço vetorial $T_{e} E$ inteiro se valer $\pi_{E}(e)=\sigma_{G}(g)$ (enquanto que, caso contrário, não é bem definida em nenhum dos seus pontos), e sua restrição a este 
espaço é uma transformação linear $L_{u_{g}}: T_{e} E \longrightarrow T_{g \cdot e} E$, pois é a composição de duas aplicações que, obviamente, são lineares:

$$
L_{u_{g}}=T_{(g, e)} \Phi_{E} \circ\left(u_{g} \circ T_{e} \pi_{E}, \mathrm{id}_{T_{e} E}\right)
$$

A compatibilidade da ação (3.24) com a estrutura de $T E$ como fibrado vetorial sobre $E$ permite transferir essa ação para todos os seus descendentes. Assim, por exemplo, obtemos uma ação de $J G$ sobre formas,

$$
\begin{aligned}
\Phi_{\bigwedge^{r} T^{*} E}: \quad J G \times_{M} \bigwedge^{r} T^{*} E & \longrightarrow \bigwedge^{r} T^{*} E \\
\left(u_{g}, \alpha_{e}\right) & \longmapsto u_{g} \cdot \alpha_{e}
\end{aligned}
$$

que restringe a uma ação de $J G$ sobre formas (parcialmente) horizontais,

$$
\begin{aligned}
\Phi_{\bigwedge_{s}^{r} T^{*} E}: \quad J G \times_{M} \bigwedge_{s}^{r} T^{*} E & \longrightarrow \bigwedge_{s}^{r} T^{*} E \\
\left(u_{g}, \alpha_{e}\right) & \longmapsto u_{g} \cdot \alpha_{e}
\end{aligned}
$$

onde, para todo ponto $e \in E, \bigwedge_{s}^{r} T_{e}^{*} E$ denota o espaço das $r$-formas sobre $T_{e} E$ que são $(r-s)$-horizontais, i.e., se anulam quando avaliadas sobre um conjunto de $r$ vetores em $T_{e} E$ entre os quais há mais de $s$ vetores verticais:

$$
\bigwedge_{s}^{r} T_{e}^{*} E=\left\{\alpha_{e} \in \bigwedge^{r} T_{e}^{*} E \mid \mathrm{i}_{v_{0}} \ldots \mathrm{i}_{v_{s}} \alpha_{e}=0 \text { para } v_{0}, \ldots, v_{s} \in V_{e} E\right\}
$$

Explicitamente, para $u_{g} \in J_{g} G, \alpha_{e} \in \bigwedge^{r} T_{e}^{*} E$ e $v_{1}, \ldots, v_{r} \in T_{g \cdot e} E$,

$$
\left(u_{g} \cdot \alpha_{e}\right)\left(v_{1}, \ldots, v_{r}\right)=\alpha_{e}\left(u_{g}^{-1} \cdot v_{1}, \ldots, u_{g}^{-1} \cdot v_{r}\right)
$$


Em termos de bisseções de $G$ e de automorfismos de $E$ por elas gerados, conforme a equação (2.74), podemos entender a ação (3.24) como a derivada do "push-forward" de curvas por automorfismos, pois para qualquer bisseção $\beta$ de $G$ e qualquer curva $\gamma$ em $E$, podemos tomar $e=\gamma(0), x=\pi_{E}(e), g=\beta(x)$ e concluir que se

$$
u_{g}=T_{x} \beta \quad \text { e } \quad v_{e}=\left.\frac{d}{d t} \gamma(t)\right|_{t=0}
$$

então

$$
u_{g} \cdot v_{e}=\left.\frac{d}{d t} \Pi_{E}(\beta)(\gamma(t))\right|_{t=0}
$$

Essa observação mostra que, e por que, a ação (3.24) é a chave para entender o que significa "invariância" de campos tensoriais sob a ação de um grupoide de Lie: dado um fibrado $E$ sobre uma variedade base $M$ munido de uma ação de um grupoide de Lie G sobre a mesma variedade base $M$, a invariância de um campo tensorial sobre o espaço total se refere à ação induzida do grupoide dos jatos $J G$ de $G$ sobre o fibrado tangente TE de $E$ e os seus descendentes, possivelmente mediante restrição a um subgrupoide adequado de $J G$. Em particular, tratando-se de uma forma diferencial $\alpha$ de grau $r$, digamos, a condição de invariância sob $\tilde{G}$, onde $\tilde{G}$ é ao mesmo tempo um subfibrado de $J G$ (como fibrado sobre $G$ ) e um subgrupoide de Lie de $J G$ (como grupoide de Lie sobre $M$ ), afirma que, para quaisquer pontos $g \in G$ e $e \in E$ tais que $\sigma(g)=\pi(e)$, qualquer 1-jato $u_{g} \in \tilde{G}_{g} \subset J_{g} G$ e quaisquer vetores tangentes $v_{1}, \ldots, v_{r} \in T_{e} E$, vale ${ }^{2}$

$$
\alpha_{g \cdot e}\left(u_{g} \cdot v_{1}, \ldots, u_{g} \cdot v_{r}\right)=\alpha_{e}\left(v_{1}, \ldots, v_{r}\right) .
$$

Observamos que essa condição é uma versão "não holônoma" da condição usual para caracterizar invariância, a saber: se $\alpha$ é invariante neste sentido, então para toda bisseção $\beta$ de $G$ cujo 1-jato toma valores em $\tilde{G}, \alpha$ é invariante sob "pull-back" pelo automorfismo correspondente $\Pi_{E}(\beta)$ de $E$ definido na equação (2.74). No entanto, a afirmação recíproca requer uma hipótese adicional sobre $\tilde{G}$ :

Definição 3.1 Sejam $G$ um grupoide de Lie sobre uma variedade base $M$ e $\tilde{G}$ um subgrupoide de Lie do seu grupoide dos jatos JG que, assim como este, também é um fibrado sobre $G$. Dizemos que $\tilde{G}$ é holônomo se para todo $u_{g} \in \tilde{G}_{g}$, existe uma bisseção $\beta$ de $G, \beta \in \operatorname{Bis}(G)$, tal que $\beta(x)=g, T_{x} \beta=u_{g}$ e $T_{y} \beta \in \tilde{G}_{\beta(y)}$ para todo $y \in M$.

Então vale

\footnotetext{
${ }^{2} \mathrm{~A}$ mesma definição se aplica a qualquer campo tensorial totalmente covariante, seja qual for seu grau de simetria.
} 
Proposição 3.1 Sejam E um fibrado sobre uma variedade base $M$, munido de uma ação de um grupoide de Lie $G$ sobre a mesma variedade base $M$, e $\tilde{G}$ um subgrupoide de Lie do seu grupoide dos jatos JG que, assim como este, também é um fibrado sobre $G$. Então denotando por $\operatorname{Bis}(G, \tilde{G})$ o subgrupo de $\operatorname{Bis}(G)$ das bisseções de $G$ cujo 1-jato toma valores em $\tilde{G}$, uma forma diferencial $\alpha \in \Omega^{r}(E)$ que é $\tilde{G}$-invariante satisfaz

$$
\Pi_{E}(\beta)^{*} \alpha=\alpha \quad \text { para todo } \beta \in \operatorname{Bis}(G, \tilde{G})
$$

e reciprocamente, se $\tilde{G}$ for holônomo, uma forma diferencial $\alpha \in \Omega^{r}(E)$ que satisfaz a condição (3.36) é $\tilde{G}$-invariante.

Demonstração: Para verificar essas afirmações, basta explicitar o lado esquerdo da equação (3.36): dados pontos $x \in M$ e $e \in E$ tais que $\pi(e)=x$, assim como vetores $v_{1}, \ldots, v_{r} \in T_{e} E$ e uma bisseção $\beta$ de $G$ tal que $\beta(x)=g$, temos $\Pi_{E}(\beta)(e)=g \cdot e$ e, usando a equação (3.25),

$$
\begin{aligned}
& \left(\Pi_{E}(\beta)^{*} \alpha\right)_{e}\left(v_{1}, \ldots, v_{r}\right)=\left(\left(\Phi_{E} \circ\left(\beta \circ \pi, \mathrm{id}_{E}\right)\right)^{*} \alpha\right)_{e}\left(v_{1}, \ldots, v_{r}\right) \\
& =\alpha_{g \cdot e}\left(T_{(g, e)} \Phi_{E}\left(\left(T_{x} \beta \circ T_{e} \pi\right)\left(v_{1}\right), v_{1}\right), \ldots, T_{(g, e)} \Phi_{E}\left(\left(T_{x} \beta \circ T_{e} \pi\right)\left(v_{r}\right), v_{r}\right)\right) \\
& =\alpha_{g \cdot e}\left(T_{x} \beta \cdot v_{1}, \ldots, T_{x} \beta \cdot v_{r}\right)
\end{aligned}
$$

Então fazendo $T_{x} \beta=u_{g}$, concluímos que a condição (3.35) implica a condição (3.36) e, se $\tilde{G}$ for holônomo, se tornam equivalentes.

Desta equivalência, segue diretamente que, pelo menos no caso holônomo, a propriedade de invariância é preservada sob o operador de derivada exterior:

Proposição 3.2 Sejam $E$ um fibrado sobre uma variedade base $M$, munido de uma ação de um grupoide de Lie $G$ sobre a mesma variedade base $M$, e $\tilde{G}$ um subgrupoide de Lie do seu grupoide dos jatos JG que, assim como este, também é um fibrado sobre G. Se $\tilde{G}$ for holônomo e se a for uma forma diferencial sobre $E$ que é $\tilde{G}$-invariante, sua derivada exterior da também é G-invariante.

\subsection{Formas Estruturais Invariantes}

Objetos centrais no formalismo hamiltoniano covariante em teoria de campos são a forma multicanônica $\theta$ e a forma multissimplética $\omega=-d \theta$ sobre o "espaço de multifase", 
que podem ser consideradas os objetos análogos à forma canônica $\theta$ e à forma simplética $\omega=-d \theta$ sobre o espaço de fase da mecânica hamiltoniana no caso em que este for o fibrado cotangente $T^{*} Q$ de algum espaço de configuração $Q$. Como já foi mencionado na introdução, em teoria de campos este "espaço de multifase" vêm em duas versões: partindo de um fibrado de configuração $E$ sobre $M$ (cujas seções são os campos originais da teoria), temos o "espaço de multifase comum", que é o dual linear torcido $\vec{J}{ }^{\circledast} E$ do fibrado dos jatos linearizados $\overrightarrow{J E}$ de $E$, e o "espaço de multifase estendido", que é o dual afim torcido $J^{\circledast} E$ do fibrado dos jatos $J E$ de $E$, sendo o segundo um fibrado em linhas sobre o primeiro e a hamiltoniana uma seção $\mathcal{H}: \vec{J}^{\circledast} E \longrightarrow J^{\circledast} E$ deste. As formas estruturais da geometria multissimplética são então a $n$-forma multicanônica "cinemática" $\theta$ e a $(n+1)$-forma multissimplética "cinemática" $\omega$ sobre $J^{\circledast} E$, as quais, mediante "pullback" pela hamiltoniana, dão origem à $n$-forma multicanônica "dinâmica" $\theta_{\mathcal{H}}=\mathcal{H}^{*} \theta$ e à $(n+1)$-forma multissimplética "dinâmica" $\omega_{\mathcal{H}}=\mathcal{H}^{*} \omega$ sobre $\vec{J}{ }^{\circledast} E .{ }^{3}$ A nossa meta nesta seção será investigar a invariância destas formas estruturais sob as pertinentes ações induzidas por uma ação de um grupoide de Lie $G$ sobre $M$.

Começando pelas formas "cinemáticas" $\theta$ e $\omega$, precisamos primeiro rever brevemente a sua construção - notando que, assim como na mecânica em fibrados cotangentes, vale $\omega=-d \theta$, de modo que podemos nos concentrar na primeira.

A construção da forma $\theta$ basea-se na representação do fibrado $J^{\circledast} E$ como um fibrado de formas sobre $E$. Mais exatamente, é bem conhecido [10] que existe um isomorfismo estrito canônico

$$
J^{\circledast} E \cong \Lambda
$$

de fibrados vetoriais sobre $E$, onde

$$
\Lambda=\bigwedge_{1}^{n} T^{*} E
$$

é o fibrado das $n$-formas sobre $E$ que são $(n-1)$-horizontais, i.e., que se anulam sob contração com dois vetores verticais. Explicitamente, este isomorfismo associa a cada $n$-forma $\alpha_{e} \in \bigwedge_{n}^{1} T_{e}^{*} E$ um cojato torcido $\Phi\left(\alpha_{e}\right) \in J_{e}^{\circledast} E$ que associa a qualquer jato $u_{e} \in J_{e} E$ o "pull-back" de $\alpha_{e}$ por $u_{e}$ :

$$
\left\langle\Phi\left(\alpha_{e}\right), u_{e}\right\rangle=u_{e}^{*} \alpha_{e}
$$

Inspecionando as equações (3.6), (3.15), (3.25) e (3.32), verifica-se então que para qualquer $u_{g} \in J_{g} G \operatorname{com} \sigma_{g}(g)=\pi_{E}(e)$, vale

$$
\Phi\left(u_{g} \cdot \alpha_{e}\right)=u_{g} \cdot \Phi\left(\alpha_{e}\right) .
$$

\footnotetext{
${ }^{3}$ Lembramos que aqui, $n=\operatorname{dim} M$.
} 
De fato, pondo $x=\sigma_{G}(g)=\pi_{E}(e)$ e $y=\tau_{G}(g)=\pi_{E}(g \cdot e)$ como de costume, então para qualquer jato $u_{g \cdot e} \in J_{g \cdot e} E$ e quaisquer vetores $h_{x, 1}, \ldots, h_{x, n} \in T_{x} M$ e $h_{y, 1}, \ldots, h_{y_{n}} \in T_{y} M$ $\operatorname{com} h_{y, i}=\left(T_{g} \tau_{G} \circ u_{g}\right)\left(h_{x, i}\right)$ e $h_{x, i}=\left(T_{g^{-1}} \tau_{G} \circ u_{g}^{-1}\right)\left(h_{y, i}\right)$, temos

$$
\begin{aligned}
\langle\Phi & \left.\left(u_{g} \cdot \alpha_{e}\right), u_{g \cdot e}\right\rangle\left(h_{y, 1}, \ldots, h_{y, n}\right) \\
= & \left(u_{g \cdot e}^{*}\left(u_{g} \cdot \alpha_{e}\right)\right)\left(h_{y, 1}, \ldots, h_{y, n}\right) \\
= & \left(u_{g} \cdot \alpha_{e}\right)\left(u_{g \cdot e}\left(h_{y, 1}\right), \ldots, u_{g \cdot e}\left(h_{y, n}\right)\right) \\
= & \alpha_{e}\left(u_{g}^{-1} \cdot u_{g \cdot e}\left(h_{y, 1}\right), \ldots, u_{g}^{-1} \cdot u_{g \cdot e}\left(h_{y, n}\right)\right) \\
= & \alpha_{e}\left(T_{\left(g^{-1}, g \cdot e\right)} \Phi_{E}\left(u_{g}^{-1}\left(h_{y, 1}\right), u_{g \cdot e}\left(h_{y, 1}\right)\right), \ldots, T_{\left(g^{-1}, g \cdot e\right)} \Phi_{E}\left(u_{g}^{-1}\left(h_{y, n}\right), u_{g \cdot e}\left(h_{y, n}\right)\right)\right) \\
= & \alpha_{e}\left(\left(T_{\left(g^{-1}, g \cdot e\right)} \Phi_{E} \circ\left(u_{g}^{-1}, u_{g \cdot e}\right) \circ\left(T_{g^{-1}} \tau_{G} \circ u_{g}^{-1}\right)^{-1}\right)\left(h_{x, 1}\right), \ldots,\right. \\
& \quad\left(T_{\left(g^{-1}, g \cdot e\right)} \Phi_{E} \circ\left(u_{g}^{-1}, u_{g \cdot e} \circ\left(T_{g^{-1}} \tau_{G} \circ u_{g}^{-1}\right)^{-1}\right)\left(h_{x, n}\right)\right) \\
= & \alpha_{e}\left(\left(u_{g}^{-1} \cdot u_{g \cdot e}\right)\left(h_{x, 1}\right), \ldots,\left(u_{g}^{-1} \cdot u_{g \cdot e}\right)\left(h_{x, n}\right)\right) \\
= & \left(\left(u_{g}^{-1} \cdot u_{g \cdot e}\right)^{*} \alpha_{e}\right)\left(h_{x, 1}, \ldots, h_{x, n}\right) \\
= & \left(\left(T_{g} \tau \circ u_{g}\right)^{-1^{*}}\left\langle\Phi\left(\alpha_{e}\right), u_{g}^{-1} \cdot u_{g \cdot e}\right\rangle\right)\left(h_{y, 1}, \ldots, h_{y, n}\right) \\
= & \left\langle u_{g} \cdot \Phi\left(\alpha_{e}\right), u_{g \cdot e}\right\rangle\left(h_{y, 1}, \ldots, h_{y, n}\right) .
\end{aligned}
$$

Portanto, temos a seguinte

Proposição 3.3 Seja E um fibrado sobre uma variedade base $M$, munido de uma ação de um grupoide de Lie $G$ sobre a mesma variedade base $M$. Então o isomorfismo estrito canônico (3.37) é JG-equivariante.

Tal fato torna-se útil porque, através do isomorfismo (3.37), a forma multicanônica $\theta$ sobre $J^{\circledast} E$ corresponde biunivocamente a uma forma multicanônica $\theta_{\Lambda}$ sobre $\Lambda$, sendo que é esta que pode ser definida por uma fórmula simples e explícita: denotando a projeção canônica de $\Lambda$ sobre $E$ (que confere a $\Lambda$ a estrutura de fibrado vetorial) por $\pi_{\Lambda}$, temos, para $\alpha_{e} \in \Lambda_{e}$ e $w_{1}, \ldots, w_{n} \in T_{\alpha_{e}} \Lambda$

$$
\left(\theta_{\Lambda}\right)_{\alpha_{e}}\left(w_{1}, \ldots, w_{n}\right)=\alpha_{e}\left(T_{\alpha_{e}} \pi_{\Lambda}\left(w_{1}\right), \ldots, T_{\alpha_{e}} \pi_{\Lambda}\left(w_{1}\right)\right) .
$$

Posto isso, podemos provar o seguinte resultado que garante a invariância da forma multicanônica $\theta$ pela ação de $J(J G, H(J G))=\bar{J}^{2} G$, definido no Apêndice A. 
Teorema 3.1 Seja E um fibrado sobre uma variedade base $M$ munido de uma ação de um grupoide de Lie $G$ sobre a mesma variedade base $M$, e considere as ações induzidas de $J G$ sobre o dual afim torcido $J^{\circledast} E$ de $J E$ e de $J(J G, H(J G))=\bar{J}^{2} G \subset J(J G)$ sobre seu fibrado tangente e seus descendentes. Então a forma multicanônica $\theta$ sobre $J^{\circledast} E$ é $J(J G, H(J G))$-invariante.

Demonstração: Inicialmente, lembramos que a ação induzida de $J G$ sobre $T E$ recobre a ação original de $G$ sobre $E$ (veja o diagrama (3.28)), e o mesmo vale para a ação induzida de $J G$ sobre $\Lambda$ (veja as equações (3.29) e (3.30)), ou seja, o diagrama

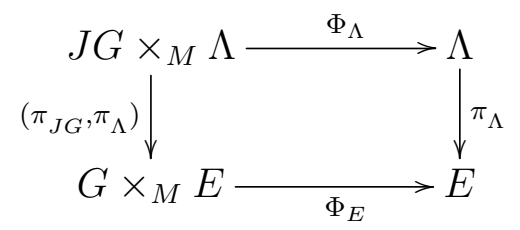

comuta: vale

$$
\pi_{\Lambda} \circ \Phi_{\Lambda}=\Phi_{E} \circ\left(\pi_{J G}, \pi_{\Lambda}\right) .
$$

Portanto, dados $u_{g} \in J_{g} G$ e $\alpha_{e} \in \Lambda_{e} \operatorname{com} \sigma_{G}(g)=\pi_{E}(e)$ e, ainda, $u_{u_{g}}^{\prime} \in J(J G, H(J G))$ e $w \in T_{\alpha_{e}} \Lambda$, temos, conforme a equação (3.25),

$$
\begin{aligned}
T_{u_{g} \cdot \alpha_{e}} \pi_{\Lambda}\left(u_{u_{g}}^{\prime} \cdot w\right) & =\left(T_{u_{g} \cdot \alpha_{e}} \pi_{\Lambda} \circ T_{\left(u_{g}, \alpha_{e}\right)} \Phi_{\Lambda}\right)\left(\left(u_{u_{g}}^{\prime} \circ T_{\alpha_{e}}\left(\pi_{E} \circ \pi_{\Lambda}\right)\right)(w), w\right) \\
& =\left(T_{(g, e)} \Phi_{E} \circ\left(T_{u_{g}} \pi_{J G}, T_{\alpha_{e}} \pi_{\Lambda}\right)\right)\left(\left(u_{u_{g}}^{\prime} \circ T_{\alpha_{e}}\left(\pi_{E} \circ \pi_{\Lambda}\right)\right)(w), w\right) \\
& =T_{(g, e)} \Phi_{E}\left(\left(T_{u_{g}} \pi_{J G} \circ u_{u_{g}}^{\prime} \circ T_{\alpha_{e}}\left(\pi_{E} \circ \pi_{\Lambda}\right)\right)(w), T_{\alpha_{e}} \pi_{\Lambda}(w)\right) \\
& =T_{(g, e)} \Phi_{E}\left(\left(u_{g} \circ T_{e} \pi_{E}\right)\left(T_{\alpha_{e}} \pi_{\Lambda}(w)\right), T_{\alpha_{e}} \pi_{\Lambda}(w)\right) \\
& =u_{g} \cdot\left(T_{\alpha_{e}} \pi_{\Lambda}(w)\right) .
\end{aligned}
$$

Assim, para $w_{1}, \ldots, w_{n} \in T_{\alpha_{e}} \Lambda$, vale

$$
\begin{aligned}
& \left(\theta_{\Lambda}\right)_{u_{g} \cdot \alpha_{e}}\left(u_{u_{g}}^{\prime} \cdot w_{1}, \ldots, u_{u_{g}}^{\prime} \cdot w_{n}\right) \\
& =\left(u_{g} \cdot \alpha_{e}\right)\left(T_{u_{g} \cdot \alpha_{e}} \pi_{\Lambda}\left(u_{u_{g}}^{\prime} \cdot w_{1}\right) \ldots, T_{u_{g} \cdot \alpha_{e}} \pi_{\Lambda}\left(u_{u_{g}}^{\prime} \cdot w_{n}\right)\right) \\
& =\left(u_{g} \cdot \alpha_{e}\right)\left(u_{g} \cdot\left(T_{\alpha_{e}} \pi_{\Lambda}\left(w_{1}\right)\right) \ldots, u_{g} \cdot\left(T_{\alpha_{e}} \pi_{\Lambda}\left(w_{n}\right)\right)\right) \\
& =\alpha_{e}\left(T_{\alpha_{e}} \pi_{\Lambda}\left(w_{1}\right) \ldots, T_{\alpha_{e}} \pi_{\Lambda}\left(w_{n}\right)\right) \\
& =\left(\theta_{\Lambda}\right)_{\alpha_{e}}\left(w_{1}, \ldots, w_{n}\right) .
\end{aligned}
$$

Para completar a demonstração, ainda precisamos transferir a afirmação de invariância da forma $\theta_{\Lambda}$ sobre $\Lambda$ para a forma $\theta$ sobre $J^{\circledast} E$, o que se faz de maneira óbvia usando a observação a seguir. 
Observação 3.1 A noção de uma forma diferencial invariante sob a ação de um grupoide de Lie é bem comportada em relação ao "pull-back". De fato, observamos que, dado dois fibrados $E$ e $F$ munidos de ações de um grupoide de Lie $G$, todos sobre a mesma variedade base $M$, então se um morfismo estrito de fibrados sobre $M, f: E \longrightarrow F$, é $G$-invariante, sua derivada $T f: T E \longrightarrow T F$ é $J G$-invariante em relação às ações induzidas, definidas conforme a equação (3.25). Segue imediatamente que, dado qualquer subgrupoide de Lie $\tilde{G}$ de $J G$, o "pull-back" de uma forma diferencial $\tilde{G}$-invariante sobre $F$ por um morfismo estrito $G$-invariante é uma forma diferencial $\tilde{G}$-invariante sobre $E$.

Observação 3.2 A questão de qual seria o grau máximo de invariância da forma multissimplética $\omega$ sobre $J^{\circledast} E$ ainda não pôde ser respondida de forma completamente satisfatória e se encontra sob investigação. Por enquanto, está apenas claro que, no mínimo, ela é $\bar{J}^{2} G$-invariante.

O nosso próximo passo é formular o conceito de invariância da hamiltoniana no formalismo multissimplético. A definição do conceito é bem óbvia: ${ }^{4}$

Definição 3.2 Seja $E$ um fibrado sobre uma variedade base $M$ munido de uma ação de um grupoide de Lie $G$ sobre a mesma variedade base $M$, e considere as ações induzidas de $J G$ sobre o dual afim torcido $J^{\circledast} E$ de $J E$ e sobre o dual linear torcido $\vec{J}^{\circledast} E$ de $\overrightarrow{J E}$. Dado um subgrupoide de Lie $\tilde{G}$ de $J G$, dizemos que uma hamiltoniana $\mathcal{H}: \vec{J}^{\circledast} E \longrightarrow J^{\circledast} E$ é $\tilde{G}$-invariante se é equivariante em relação às ações pertinentes de $J G$, quando restritas a $\tilde{G}$, ou seja, se o seguinte diagrama comuta:

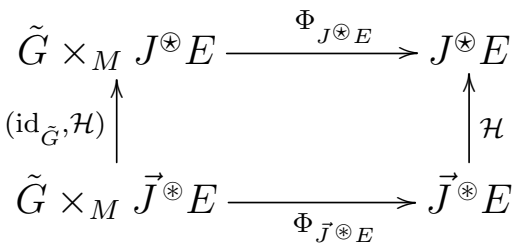

A invariância de $\mathcal{H}$ implica em invariância da forma $\theta_{\mathcal{H}}$, conforme segue:

Teorema 3.2 Seja E um fibrado sobre uma variedade base $M$ munido de uma ação de um grupoide de Lie $G$ sobre a mesma variedade base $M$, e considere as ações induzidas de $J G$ sobre o dual linear torcido $\vec{J}^{\circledast} E$ de $\vec{J} E$ e de $J(J G, H(J G))=\bar{J}^{2} G \subset J(J G)$ sobre seu fibrado tangente e seus descendentes. Considere ainda algum subgrupoide de Lie

\footnotetext{
${ }^{4}$ Usamos o termo "invariante" em um sentido amplo, o qual inclui o conceito de "equivariante" como caso especial, pois uma aplicação $f$ de um espaço $X$ para um espaço $Y$ é equivariante em relação a ações de um grupo ou grupoide $G$ sobre $X$ e $Y$ se e somente se é invariante sob a ação induzida de $G$ sobre o espaço $\operatorname{Map}(X, Y)$.
} 
$\tilde{G}$ de $J G$ e o correspondente subgrupoide de Lie $J(\tilde{G}, H(\tilde{G}))$ de $J(J G, H(J G))$. Então se uma hamiltoniana $\mathcal{H}: \vec{J}^{\circledast} E \longrightarrow J^{\circledast} E$ for $\tilde{G}$-invariante, a forma $\theta_{\mathcal{H}}$ sobre $\vec{J}^{\circledast} E$ é $J(\tilde{G}, H(\tilde{G}))$-invariante.

Demonstração: A afirmação segue imediatamente do teorema anterior em conjunto com a observação que o segue.

Observação 3.3 Novamente, a questão qual seria o grau máximo de invariância da forma $\omega_{\mathcal{H}}$ sobre $\vec{J}^{\circledast} E$ ainda não pôde ser respondida de forma completamente satisfatória e se encontra sob investigação.

\subsection{O Teorema de Noether}

Começamos por introduzir o conceito da aplicação momento no contexto deste trabalho:

Definição 3.3 Seja $E$ um fibrado sobre uma variedade base $M$ munido de uma ação de um grupoide de Lie $G$ sobre a mesma variedade base $M$, e considere a ação induzida de $J G$ sobre o dual linear torcido $\vec{J}{ }^{\circledast} E$ de $\overrightarrow{J E}$, assim como a ação infinitesimal de $A(J G)$ sobre $\vec{J}{ }^{\circledast} E$ induzida por esta. Então a aplicação momento associada a estas ações é a aplicação

$$
\begin{aligned}
j: \Gamma(A(J G)) & \longrightarrow \quad \Omega^{n-1}\left(\vec{J}^{\circledast} E\right) \\
X & \longmapsto j(X)=i_{X_{\vec{J}_{\circledast} E}} \theta_{\mathcal{H}}
\end{aligned}
$$

Teorema 3.3 (Teorema de Noether) Seja E um fibrado sobre uma variedade base $M$ munido de uma ação de um grupoide de Lie $G$ sobre a mesma variedade base $M$, e considere as ações induzidas de $J G$ sobre o dual afim torcido $J^{\circledast} E$ de $J E$ e sobre o dual linear torcido $\vec{J}{ }^{\circledast} E$ de $\overrightarrow{J E}$. Dados um subgrupoide de Lie $\tilde{G}$ de $J G$ e uma hamiltoniana $\mathcal{H}: \vec{J}^{\circledast} E \longrightarrow J^{\circledast} E$ que é $\tilde{G}$-invariante, então para todo $X \in \Gamma(A \tilde{G})$ e toda seção $\phi$ de $\vec{J}{ }^{\circledast} E$ satisfazendo as equações de movimento, ou seja, as equações de De Donder-Weyl, a correspondente corrente de Noether $\phi^{*} j(X) \in \Omega^{n-1}(M)$ é conservada:

$$
d\left[\phi^{*} j(X)\right]=0 .
$$

Demonstração: Fixamos $X \in \Gamma(A \tilde{G})$. Então para $\phi \in \Gamma\left(\vec{J}^{\circledast} E\right)$, vale

$$
\begin{aligned}
d\left[\phi^{*} j(X)\right] & =d\left[\phi^{*}\left(i_{X_{\vec{J} \circledast E}} \theta_{\mathcal{H}}\right)\right]=\phi^{*} d\left(i_{X_{\vec{J} \circledast E}} \theta_{\mathcal{H}}\right) \\
& =\phi^{*}\left(\mathcal{L}_{X_{\vec{J} \circledast E}} \theta_{\mathcal{H}}\right)+\phi^{*}\left(i_{X_{\vec{J} \circledast E}} \omega_{\mathcal{H}}\right) .
\end{aligned}
$$


Afirmação 1: Vale

$$
\mathcal{L}_{X_{\vec{J} \circledast E}} \theta_{\mathcal{H}}=0 .
$$

De fato, $X$ gera um subgrupo a um parâmetro $\exp (t X)$ de bisseções de $\tilde{G}$ e então $X_{\vec{J}{ }^{\oplus}}$ gera o subgrupo a um parâmetro $\Pi_{\vec{J}^{\circledast} E}(\exp (t X))$ de automorfismos de $\vec{J}{ }^{\circledast} E$. Como, por hipótese, $\mathcal{H}$ é $\tilde{G}$-invariante e portanto, conforme o Teorema 3.2 , $\theta_{\mathcal{H}}$ é $J(\tilde{G}, H(\tilde{G}))$ invariante, segue que

$$
\mathcal{L}_{X_{\vec{J}^{\oplus E}}} \theta_{\mathcal{H}}=\left.\frac{d}{d t} \Pi_{\vec{J}^{\circledast E}}(\exp (t X))^{*} \theta_{\mathcal{H}}\right|_{t=0}=\left.\frac{d}{d t} \theta_{\mathcal{H}}\right|_{t=0}=0 .
$$

Afirmação 2: Se $\phi$ é solução das equações de De Donder-Weyl, vale

$$
\phi^{*}\left(i_{X_{\vec{J} \circledast E}} \omega_{\mathcal{H}}\right)=0 .
$$

Isso é um resultado clássico e, na verdade, vale uma afirmação muito mais forte: $\phi$ é solução das equações de De Donder-Weyl se e somente se

$$
\phi^{*} i_{Z} \omega_{\mathcal{H}}=0 .
$$

para todo campo vertical e até para todo campo vetorial projetável $Z$ sobre $\vec{J}{ }^{\circledast} E$; veja [7], por exemplo. 


\section{Apêndice A}

\section{Grupoides de Pfaff}

Introduzimos neste apêndice a noção de um grupoide de Pfaff, que é um refinamento do conceito de um grupoide de Lie no sentido de acrescentar um novo elemento estrutural, a saber, a especificação de uma distribuição, com certas propriedades, sobre o espaço total. Para formular quais são estas propriedades, precisamos primeiro da seguinte

Definição A.1 Seja $G$ um grupoide de Lie sobre uma variedade $M$, com projeção fonte $\sigma$ e projeção alvo $\tau$, e seja $D \subset T G$ uma distribuição sobre $G$. Então dizemos que $D$ é subinvolutiva se valer

$$
D^{\sigma}=D^{\tau}
$$

(onde $D^{\sigma}=D \cap V^{\sigma} G \equiv D \cap \operatorname{ker} T \sigma$ e $D^{\tau}=D \cap V^{\tau} G \equiv D \cap \operatorname{ker} T \tau$, como antes) e se esta sub-distribuição for involutiva.

Definição A.2 Um grupoide de Pfaff é um grupoide de Lie G sobre uma variedade $M$ munido de uma distribuição subinvolutiva $D \subset T G$ que também é subgrupoide de Lie do grupoide de Lie TG sobre TM.

Observação A.1 Aqui, desviamos ligeiramente da convenção adotada em [19]: como já incluímos a igualdade (A.1) na definição, um grupoide de Pfaff no sentido adotado neste trabalho corresponde a um grupoide de Lie-Pfaff no sentido de [19].

Observação A.2 A afirmação de que a distribuição $D$ de um grupoide de Pfaff seja um subgrupoide de Lie do grupoide de Lie $T G$ sobre $T M$ agrega uma série de propriedades:

1. $D$ é fechada sob a multiplicação $\mu_{T G}$ de $T G$, que é a tangente à multiplicação $\mu_{G}$ de $G$ : é a afirmação de que $D$ é multiplicativa; 
2. $D$ contém a unidade $T M$ de $T G$, ou mais precisamente, a imagem de $T M$ sob a unidade $1_{T G}$ de $T G$, que é a tangente à unidade $1_{G}$ de $G$;

3. $D$ é fechada sob a inversão $\iota_{T G}$ de $T G$, que é a tangente à inversão $\iota_{G}$ de $G$;

4. Sendo que, por hipótese, $D$ é subfibrado vetorial de $T G$, i.e., tem posto constante, o mesmo vale para $D^{\sigma}=D^{\tau}$. Para provar isso, usamos apenas as propriedades 1 . e 2. enunciadas anteriormente, abreviando $1_{G}$ para 1 e retendo a definição original $1_{T G}=T 1$. De fato, para todo ponto $x$ de $M$, temos a decomposição direta $T_{1_{x}} G=$ $V_{1_{x}}^{\sigma} G \oplus T_{x} 1\left(T_{x} M\right)$, a qual, tendo em vista que conforme o item 2 ., o espaço $D_{1_{x}}$ contém o segundo somando, implica a decomposição direta $D_{1_{x}}=D_{1_{x}}^{\sigma} \oplus T_{x} 1\left(T_{x} M\right)$, de modo que a dimensão de $D_{1_{x}}^{\sigma}$ não depende de $x$. Agora, dado um ponto $g$ de $G$ qualquer com $\sigma(g)=x$ e $\tau(g)=y$, o isomorfismo linear $T_{1_{y}} R_{g}: V_{1_{y}}^{\sigma} G \longrightarrow V_{g}^{\sigma} G$ que é a tangente, em $1_{y}$, ao difeomorfismo $R_{g}: G_{y} \longrightarrow G_{x}$ de translação à direita por $g$, leva $D_{1_{y}}^{\sigma}$ em $D_{g}^{\sigma}$, devido à condição de multiplicatividade exigida no item 1., mostrando que a dimensão de $D_{g}^{\sigma}$ não depende de $g$. Segue que, ainda nestas condições, $D$ é $\sigma$-transversal e $\tau$-transversal, isto é, vale

$$
D+V^{\sigma} G=T G=D+V^{\tau} G
$$

sendo que estas somas não são diretas, uma vez que, por hipótese, também vale

$$
D \cap V^{\sigma} G=D^{\sigma}=D^{\tau}=D \cap V^{\tau} G .
$$

Observação A.3 Dado um grupoide de Lie $G$ sobre uma variedade $M$ e uma distribuição subinvolutiva $D \subset T G$ sobre $G$, é suficiente verificar os itens 1., 2. e 3. da observação anterior para poder concluir que se trata de um grupoide de Pfaff. Essa afirmação não é trivial na medida em que um subgrupoide abstrato de um grupoide de Lie não é necessariamente um subgrupoide de Lie, mas vale no presente caso porque a última afirmação do item 4. da observação anterior assegura que as projeções fonte $\left.T \sigma\right|_{D}: D \longrightarrow T M$ e alvo $\left.T \tau\right|_{D}: D \longrightarrow T M$ são sobrejetoras, e sendo morfismos de fibrados vetoriais sobre as projeções fonte $\sigma: G \longrightarrow M$ e alvo $\tau: G \longrightarrow M$ originais, também são submersões, o que é essencial para que $D$ possa se tornar um grupoide de Lie sobre $T M$ no seu próprio direito.

De passagem, notamos que noções básicas tais como as de morfismo entre grupoides, de subgrupoide, de produto fibrado de grupoides e de bisseção podem ser facilmente adaptados de grupoides de Lie para grupoides de Pfaff; nós omitiremos os detalhes pois não serão essenciais para o que segue. Mencionamos apenas que uma bisseção de um grupoide de Pfaff $(G, D)$ sobre uma variedade base $M$ é uma aplicação $\beta: M \longrightarrow G$ que, além de ser uma bisseção de $G$ (i.e., vale $\sigma \circ \beta=\operatorname{id}_{M}$ e $\left.\tau \circ \beta \in \operatorname{Diff}(M)\right)$ também 
satisfaz $T \beta(T M) \subset D$. O conjunto de todas as bisseções de $(G, D)$ será denotado por $\operatorname{Bis}(G, D)$ e forma um subgrupo de $\operatorname{Bis}(G)$.

A cada grupoide de Pfaff podemos associar o seu grupoide dos jatos:

Definição A.3 Seja $(G, D)$ um grupoide de Pfaff sobre uma variedade base $M$. Então o seu grupoide dos jatos $J(G, D)$ é o subgrupoide de Lie do grupoide dos jatos $J G$ de $G$ definido por:

$$
J_{g}(G, D)=\left\{u_{g} \in J_{g} G \mid \operatorname{im} u_{g} \subset D_{g}\right\}
$$

Com este conceito, temos uma ferramenta para lidar com simetrias oriundas de alguma ação de um grupoide de Lie $G$ mas onde as transformações de simetria que são permitidas devem satisfazer certas restrições sobre as suas derivadas que podem não ser induzidas, por diferenciação, a partir de correspondentes restrições sobre os seus valores. Isso corresponde a considerar situações onde há invariância sob a ação não do inteiro grupoide dos jatos $J G$ do grupoide de Lie original $G$, mas apenas de algum subgrupoide de Lie $\tilde{G}$, que pode chegar a não admitir nenhuma seção holônoma, e a ideia de usar grupoides de Pfaff no contexto do presente trabalho deriva da observação de que uma maneira de construir tais subgrupoides (se bem que não a única) é considerar o grupoide dos jatos $J(G, D)$ de algum grupoide de Pfaff $(G, D)$.

Para podermos ser mais específicos, precisamos discutir o que talvez seja o mais importante exemplo de um grupoide de Pfaff: o grupoide dos jatos $J G$ de um grupoide de Lie $G$ qualquer é sempre um grupoide de Pfaff, e sem apreciar esta estrutura adicional, não é possível entender por completo o significado da invariância da forma multissimplética em teoria de campos.

Exemplo A.1 (Grupoide tangente horizontal) Um exemplo não-trivial e de suma importância para o presente trabalho é obtido através da seguinte construção: dado qualquer grupoide de Lie $G$ sobre qualquer variedade base $M$, passamos primeiro para o seu grupoide de jatos, que ainda é um grupoide de Lie $J G$ sobre a mesma variedade base $M$, e daí para o grupoide tangente deste, que agora é um grupoide de Lie $T(J G)$ sobre o fibrado tangente $T M$ de $M$. Ocorre que este último contém uma distribuição canônica $H(J G)$ que é o exato análogo da distribuição horizontal universal para fibrados construída na Seção 2.1 e que, mais uma vez por abuso de linguagem, chamaremos a distribuição horizontal universal do grupoide de Lie $G$. Explicitamente, para uma melhor visualização das 
devidas adaptações de notação, considere o diagrama comutativo

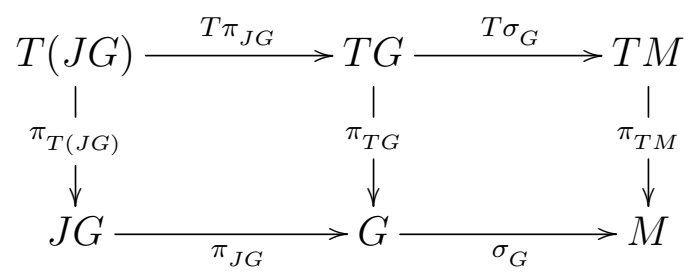

onde as flechas verticais são as projeções canônicas dos respectivos fibrados tangente; então conforme as equações (2.35)-(2.38), temos, para $g \in G$ e $u_{g} \in J_{g} G$,

$$
H_{u_{g}} G=\operatorname{ker}\left(\mathrm{id}_{T_{g} G}-u_{g} \circ T_{g} \sigma_{G}\right) \subset T_{g} G
$$

$\mathrm{e}$

$$
H_{u_{g}}(J G)=\operatorname{ker}\left(\left(\mathrm{id}_{T_{g} G}-u_{g} \circ T_{g} \sigma_{G}\right) \circ T_{u_{g}} \pi_{J G}\right) \subset T_{u_{g}}(J G)
$$

ou ainda

$$
H_{u_{g}} G=\left\{v_{g} \in T_{g} G \mid v_{g}=\left(u_{g} \circ T_{g} \sigma_{G}\right) \cdot v_{g}\right\}
$$

e

$$
H_{u_{g}}(J G)=\left\{w_{u_{g}} \in T_{u_{g}}(J G) \mid T_{u_{g}} \pi_{J G} \cdot w_{u_{g}}=\left(u_{g} \circ T_{u_{g}} \sigma_{J G}\right) \cdot w_{u_{g}}\right\}
$$

Então é imediato que vale

$$
H_{u_{g}} G \cap V_{g}^{\sigma} G=\{0\}
$$

mas também vale

$$
H_{u_{g}} G \cap V_{g}^{\tau} G=\{0\}
$$

pois dado $v_{g} \in H_{u_{g}} G \cap V_{g}^{\tau} G$, aplicamos $T_{g} \tau_{G}$ à fórmula que define $H_{u_{g}} G$ para obter

$$
0=T_{g} \tau_{G} \cdot v_{g}=\left(T_{g} \tau_{G} \circ u_{g} \circ T_{g} \sigma_{G}\right) \cdot v_{g}
$$

e portanto $T_{g} \sigma_{G} \cdot v_{g}=0$ pois $T_{g} \tau_{G} \circ u_{g}$ é um isomorfismo linear, e agora podemos aplicar a igualdade anterior para concluir que $v_{g}=0$. Tomando a imagem inversa sob $T \pi_{J G}$, obtemos

$$
H(J G)^{\sigma} \equiv H(J G) \cap V^{\sigma}(J G)=V^{\pi}(J G)
$$

e também

$$
H(J G)^{\tau} \equiv H(J G) \cap V^{\tau}(J G)=V^{\pi}(J G)
$$

$\operatorname{com} V^{\pi}(J G)=\operatorname{ker} T \pi_{J G}$, e como este, sendo o fibrado vertical de um fibrado, é obviamente involutivo, segue que $H(J G)$ é subinvolutivo. Resta provar que $H(J G)$ é subgrupoide de $T(J G)$. Para tanto, observamos primeiro que temos nada menos do que quatro grupoides de Lie envolvidos, relacionados entre eles por vários morfismos, pois 
além dos grupoides $G$ e $J G$, ambos sobre $M$, temos os grupoides $T G$ e $T(J G)$, ambos sobre $T M$, e já sabendo que, conforme foi estabelecido no Exemplo $2.10, \pi_{J G}: J G \longrightarrow G$ é um morfismo estrito de grupoides de Lie (sobre $M$ ), mostraremos que sua aplicação tangente $T \pi_{J G}: T(J G) \longrightarrow T G$ também é um morfismo estrito de grupoides de Lie (sobre $T M$ ). De fato, dados $g_{1}, g_{2} \in G, u_{1} \in J_{g_{1}} G, u_{2} \in J_{g_{2}} G, w_{1} \in T_{u_{1}}(J G)$ e $w_{2} \in T_{u_{2}}(J G)$ tais que $\sigma_{G}\left(g_{2}\right)=\tau_{G}\left(g_{1}\right), \sigma_{J G}\left(u_{2}\right)=\tau_{J G}\left(u_{1}\right)$ e $\sigma_{T(J G)}\left(w_{2}\right)=\tau_{T(J G)}\left(w_{1}\right)$, ou seja,

$$
T_{u_{2}} \sigma_{J G} \cdot w_{2}=T_{u_{1}} \tau_{J G} \cdot w_{1},
$$

de modo que os produtos $g_{2} g_{1} \in G, u_{2} u_{1} \in J G$ e $w_{2} w_{1} \in T(J G)$ estão bem definidos, então pondo

$$
v_{i}=T_{u_{i}} \pi_{J G} \cdot w_{i}
$$

o produto $v_{2} v_{1} \in T G$ também está bem definido, e vale

$$
v_{2} v_{1}=T_{u_{2} u_{1}} \pi_{J G} \cdot\left(w_{2} w_{1}\right)
$$

Isso segue de uma aplicação elementar da regra da cadeia:

$$
\begin{aligned}
T_{u_{2} u_{1}} \pi_{J G} \cdot\left(w_{2} w_{1}\right) & =\left(T_{\mu_{J G}\left(u_{2}, u_{1}\right)} \pi_{J G} \circ T_{\left(u_{2}, u_{1}\right)} \mu_{J G}\right) \cdot\left(w_{2}, w_{1}\right) \\
& =T_{\left(u_{2}, u_{1}\right)}\left(\pi_{J G} \circ \mu_{J G}\right) \cdot\left(w_{2}, w_{1}\right) \\
& =T_{\left(u_{2}, u_{1}\right)}\left(\mu_{G} \circ\left(\pi_{J G}, \pi_{J G}\right)\right) \cdot\left(w_{2}, w_{1}\right) \\
& =T_{\left(g_{2}, g_{1}\right)} \mu_{G} \cdot\left(T_{u_{2}} \pi_{J G} \cdot w_{2}, T_{u_{1}} \pi_{J G} \cdot w_{1}\right) \\
& =\left(T_{u_{2}} \pi_{J G} \cdot w_{2}\right)\left(T_{u_{1}} \pi_{J G} \cdot w_{1}\right)
\end{aligned}
$$

Da mesma forma, segue que $T \pi_{J G}$ leva a unidade $1_{T(J G)}=T 1_{J G}$ de $T(J G)$ para a unidade $1_{T G}=T 1_{G}$ de $T G$,

$$
T \pi_{J G} \circ T 1_{J G}=T\left(\pi_{J G} \circ 1_{J G}\right)=T 1_{G}
$$

e leva inversos para inversos: dados $g \in G, u_{g} \in J_{g} G$ e $w_{u_{g}} \in T_{u_{g}}(J G)$, vale

$$
\begin{aligned}
T_{u_{g}^{-1}} \pi_{J G} \cdot w_{u_{g}}^{-1} & =\left(T_{\iota_{J G}\left(u_{g}\right)} \pi_{J G} \circ T_{u_{g}} \iota_{J G}\right) \cdot w_{u_{g}} \\
& =T_{u_{g}}\left(\pi_{J G} \circ \iota_{J G}\right) \cdot w_{u_{g}} \\
& =T_{u_{g}}\left(\iota_{G} \circ \pi_{J G}\right) \cdot w_{u_{g}} \\
& =\left(T_{g} \iota_{G} \circ T_{u_{g}} \pi_{J G}\right) \cdot w_{u_{g}} \\
& =\left(T_{u_{g}} \pi_{J G} \cdot w_{u_{g}}\right)^{-1}
\end{aligned}
$$

Sendo assim, a tarefa de provar que $H(J G)$ é subgrupoide de $T(J G)$ se reduz a provar as propriedades pertinentes para $H_{J G} G$. Para tanto, manteremos a notação introduzida acima. 
- $H(J G)$ é multiplicativo. Dados $g_{1}, g_{2} \in G, u_{1} \in J_{g_{1}} G, u_{2} \in J_{g_{2}} G, v_{1} \in T_{g_{1}} G$ e $v_{2} \in T_{g_{2}} G$ tais que $\sigma_{G}\left(g_{2}\right)=\tau_{G}\left(g_{1}\right), \sigma_{J G}\left(u_{2}\right)=\tau_{J G}\left(u_{1}\right)$ e $\sigma_{T G}\left(v_{2}\right)=\tau_{T G}\left(v_{1}\right)$, de modo que $g_{2} g_{1} \in G, u_{2} u_{1} \in J G$ e $v_{2} v_{1} \in T G$ estão bem definidos, precisamos mostrar que se $v_{1} \in H_{u_{1}} G$ e $v_{2} \in H_{u_{2}} G$, ou seja,

$$
\left(\operatorname{id}_{T_{g_{i}} G}-u_{i} \circ T_{g_{i}} \sigma_{G}\right) \cdot v_{i}=0
$$

para $i=1,2$, então $v_{2} v_{1} \in H_{u_{2} u_{1}} G$, ou seja,

$$
\left(\operatorname{id}_{T_{g_{2} g_{1}} G}-\left(u_{2} u_{1}\right) \circ T_{g_{2} g_{1}} \sigma_{G}\right) \cdot\left(v_{2} v_{1}\right)=0
$$

Isso segue de um cálculo direto, usando a regra da cadeia em conjunto com propriedades elementares das aplicações envolvidas (tais como a identidade $\left.\sigma_{G} \circ \mu_{G}=\sigma_{G} \circ \mathrm{pr}_{2}\right)$ e a definição do produto em $J G$ dada no Exemplo 2.6:

$$
\begin{aligned}
\left(u_{2} u_{1} \circ T_{g_{2} g_{1}} \sigma_{G}\right) \cdot\left(v_{2} v_{1}\right) & =\left(\mu_{J G}\left(u_{2}, u_{1}\right) \circ T_{\mu_{G}\left(g_{2}, g_{1}\right)} \sigma_{G} \circ T_{\left(g_{2}, g_{1}\right)} \mu_{G}\right) \cdot\left(v_{2}, v_{1}\right) \\
& =\left(\mu_{J G}\left(u_{2}, u_{1}\right) \circ T_{g_{1}} \sigma_{G}\right) \cdot v_{1} \\
& =\left(T_{\left(g_{2}, g_{1}\right)} \mu_{G} \circ\left(u_{2} \circ\left(T_{g_{1}} \tau_{G} \circ u_{1}\right), u_{1}\right) \circ T_{g_{1}} \sigma_{G}\right) \cdot v_{1} \\
& =\left(T_{\left(g_{2}, g_{1}\right)} \mu_{G} \circ\left(u_{2} \circ\left(T_{g_{1}} \tau_{G} \circ u_{1}\right) \circ T_{g_{1}} \sigma_{G}, u_{1} \circ T_{g_{1}} \sigma_{G}\right)\right) \cdot v_{1} \\
& =T_{\left(g_{2}, g_{1}\right)} \mu_{G} \cdot\left(\left(u_{2} \circ T_{g_{1}} \tau_{G}\right) \cdot v_{1}, v_{1}\right) \\
& =T_{\left(g_{2}, g_{1}\right)} \mu_{G} \cdot\left(\left(u_{2} \circ T_{g_{2}} \sigma_{G}\right) \cdot v_{2}, v_{1}\right) \\
& =T_{\left(g_{2}, g_{1}\right)} \mu_{G} \cdot\left(v_{2}, v_{1}\right) \\
& =v_{2} v_{1}
\end{aligned}
$$

- $H(J G)$ contém a unidade. Dados $x \in M$ e $h \in T_{x} M$, precisamos mostrar que $1_{T(J G), h} \in T_{1_{J G, x}}(J G)$ pertence a $H_{1_{J G, x}}(J G)$, i.e., que

$$
T_{1_{J G, x}} \pi_{J G} \cdot 1_{T(J G), h}=\left(T_{1_{J G, x}} \pi_{J G} \circ T_{x} 1_{J G}\right) \cdot h=T_{x} 1_{G} \cdot h \in T_{1_{G, x}} G
$$

pertence a $H_{1_{J G, x}} G$. Mas isso segue diretamente observando que a unidade $1_{J G}$ de $J G$ é o 1 -jato da unidade $1_{G}$ de $G$, e portanto

$$
\left(1_{J G, x} \circ T_{1_{G, x}} \sigma_{G}\right) \cdot\left(T_{x} 1_{G} \cdot h\right)=1_{J G, x} \cdot h=T_{x} 1_{G} \cdot h .
$$

- $H(J G)$ é fechado sob a inversão. Dados $g \in G, u_{g} \in J_{g} G$ e $v_{g} \in T_{g} G$, precisamos mostrar que se $v_{g} \in H_{u_{g}} G$, ou seja,

$$
\left(\mathrm{id}_{T_{g} G}-u_{g} \circ T_{g} \sigma_{G}\right) \cdot v_{g}=0
$$


então $v_{g}^{-1} \in H_{u_{g}^{-1}} G$, ou seja,

$$
\left(\mathrm{id}_{T_{g^{-1}} G}-u_{g}^{-1} \circ T_{g^{-1}} \sigma_{G}\right) \cdot v_{g}^{-1}=0
$$

Novamente, isso segue de um cálculo direto, usando a regra da cadeia em conjunto com propriedades elementares das aplicações envolvidas (tais como a identidade $\left.\sigma_{G}{ }^{\circ}{ }_{G}=\tau_{G}\right)$ e a definição do inverso em $J G$ dada no Exemplo 2.6:

$$
\begin{aligned}
\left(u_{g}^{-1} \circ T_{g^{-1}} \sigma_{G}\right) \cdot v_{g}^{-1} & =\left(\iota_{J G}\left(u_{g}\right) \circ T_{\iota_{G}}(g) \sigma_{G} \circ T_{g} \iota_{G}\right) \cdot v_{g} \\
& =\left(\iota_{J G}\left(u_{g}\right) \circ T_{g} \tau_{G}\right) \cdot v_{g} \\
& =\left(T_{g} \iota_{G} \circ u_{g} \circ\left(T_{g} \tau_{G} \circ u_{g}\right)^{-1} \circ T_{g} \tau_{G}\right) \cdot\left(\left(u_{g} \circ T_{g} \sigma_{G}\right) \cdot v_{g}\right) \\
& =\left(T_{g} \iota_{G} \circ u_{g} \circ T_{g} \sigma_{G}\right) \cdot v_{g} \\
& =T_{g} \iota_{G} \cdot v_{g} \\
& =v_{g}^{-1}
\end{aligned}
$$

O grupoide de Pfaff $(J G, H(J G))$ assim definido será chamado de grupoide tangente horizontal de $J G$.

Toda essa construção admite uma generalização direta quando substituirmos o grupoide de Lie $J G$ por algum subgrupoide de Lie $\tilde{G}$ que também é um fibrado sobre $G$, como no final da Seção 3.1, e a distribuição horizontal universal $H(J G)$ pela distribuição $H(\tilde{G})=\left.H(J G)\right|_{\tilde{G}} \cap T \tilde{G}$, o que leva a um grupoide de Pfaff $(\tilde{G}, H(\tilde{G}))$.

Notamos que este último exemplo constitui a "versão para grupoides" da construção da distribuição horizontal universal de um fibrado apresentada na Seção 2.1. Da mesma forma, podemos formular uma "versão para grupoides" da construção do fibrado dos jatos não holônomos de segunda ordem que também foi discutida na Seção 2.1. Brevemente, dado um grupoide de Lie $G$ sobre uma variedade base $M$, consideramos seu grupoide dos jatos $J G$ e o grupoide dos jatos deste, $J(J G)$. Como no caso dos fibrados, temos então duas projeções de $J(J G)$ para $J G, \pi_{J(J G)}$ e $J \pi_{J G}$, esta última definida por

$$
\left(J \pi_{J G}\right)_{u_{g}}\left(u_{u_{g}}^{\prime}\right)=T_{u_{g}} \pi_{J G} \circ u_{u_{g}}^{\prime} \quad \text { para } u_{g} \in J_{g} G, u_{u_{g}}^{\prime} \in J_{u_{g}}(J G),
$$

e que se enquadram em um diagrama comutativo:

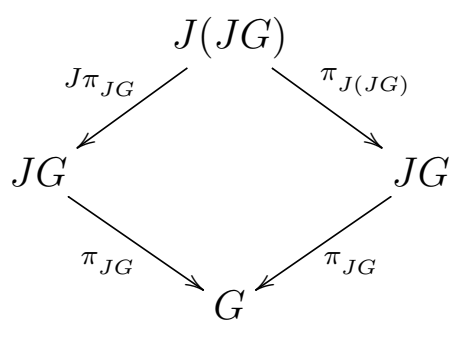


Definimos então, para cada ponto $u_{g} \in J_{g} G$,

$$
\bar{J}_{u_{g}}^{2} G=\left\{u_{u_{g}}^{\prime} \in J_{u_{g}}(J G) \mid T_{u_{g}} \pi_{J G} \circ u_{u_{g}}^{\prime}=u_{g}\right\} .
$$

Variando o ponto $u_{g}$ e observando que tanto $\pi_{J(J G)}$ como $J \pi_{J G}$ são morfismos estritos de grupoides de Lie sobre $M$, obtemos um subgrupoide de Lie $\bar{J}^{2} G$ do grupoide de Lie $J(J G)$ sobre $M$ chamado de grupoide dos jatos não holônomos de segunda ordem de $G$, e ainda como no caso dos fibrados, chegamos á conclusão de que

$$
\bar{J}^{2} G=J(J G, H(J G)) .
$$

Ainda podemos considerar uma generalização desta construção quando substituirmos o grupoide $J G$ por algum subgrupoide $\tilde{G}$, como no final da Seção 3.1, e considerar o grupoide dos jatos $J(\tilde{G}, H(\tilde{G}))$ do grupoide de Pfaff $(\tilde{G}, H(\tilde{G}))$. 


\section{Referências Bibliográficas}

[1] R. Abraham \& J.E. Marsden, Foundations of Mechanics, 2nd edition, BenjaminCummings, Reading 1978.

[2] J.F. Cariñena, M. Crampin \& L.A. Ibort, On the Multisymplectic Formalism for First Order Field Theories, Diff. Geom. Appl. 1 (1991) 345-374.

[3] M. Crainic \& R.L. Fernandes, Exotic Characteristic Classes of Lie Algebroids, in: Quantum Field Theory and Noncommutative Geometry, eds.: U. Carow-Watamura, Y. Maeda \& S. Watamura, Lecture Notes in Physics 662, Springer-Verlag, Berlin 2005.

[4] M. Crainic, M.A. Salazar \& I. Struchiner, Multiplicative Forms and Spencer Operators, Preprint arXiv:1210.2277v1.

[5] M. Forger \& J. Kellendonk, Classical BRST Cohomology and Invariant Functions on Constraint Manifolds I, Commun. Math. Phys. 143 (1992) 235-251.

[6] M. Forger \& H. Römer, Currents and the Energy-Momentum Tensor in Classical Field Theory: a Fresh Look at an Old Problem, Ann. Phys. 309 (2004) 306-389.

[7] M. Forger \& S.V. Romero, Covariant Poisson Brackets in Geometric Field Theory, Commun. Math. Phys. 256 (2005) 375-410.

[8] M. Forger \& B.L. Soares, Local Symmetries in Gauge Theories in a FiniteDimensional Setting, J. Geom. Phys. 62 (2012) 1925-1938.

[9] M. Forger \& S.Z. Yepes, Lagrangian Distributions and Connections in Multisymplectic and Polysymplectic Geometry, Diff. Geom. Appl. 31 (2013) 775-807.

[10] M.J. Gotay, J. Isenberg, J.E. Marsden and R. Montgomery, Momentum Maps and Classical Relativistic Fields. Part I: Covariant Field Theory, Preprint arXiv:physics/9801019v2. 
[11] M. Jotz \& C. Ortiz, Foliated Groupoids and Their Infinitesimal Data, Preprint arXiv: $1109.4515 \mathrm{v} 5$.

[12] S. Kobayashi \& K. Nomizu, Foundations of Differential Geometry, Vol. 1, Interscience, New York 1963.

[13] I. Kolár, P.W. Michor \& J. Slovák, Natural Operations in Differential Geometry, Springer-Verlag, Berlin 1993.

[14] Y. Kosmann-Schwarzbach \& K.C.H. Mackenzie, Differential Operators and Actions of Lie Algebroids, Preprint arXiv:math/0209337v2.

[15] S. Lang, Differential and Riemannian Manifolds, 3rd edition, Springer-Verlag, Berlin 1995.

[16] K.C.H. Mackenzie, General Theory of Lie Groupoids and Lie Algebroids, Cambridge University Press, Cambridge 2005.

[17] L.H.P. Pêgas, Grupoides de Lie e o Teorema de Noether na Formulação Lagrangiana da Teoria Clássica de Campos, Tese de Doutorado, IME-USP, Setembro de 2014.

[18] J. Pradines, Fibres vectoriels doubles et calcul des jets non holonomes, Esquisses Mathématiques [Mathematical Sketches] 29, Université d'Amiens U.E.R. de Mathématiques, Amiens 1977.

[19] M.A. Salazar, Pfaffian Groupoids, Tese de Doutorado, Universiteit Utrecht, Maio de 2013, Preprint arXiv:1306:1164v2.

[20] D.J. Saunders, The Geometry of Jet Bundles, Cambridge University Press, Cambridge 1989 .

[21] A.C. da Silva, Lectures on Symplectic Geometry, Lecture Notes in Mathematics 1764, Springer-Verlag, Berlin 2001.

[22] B.L. Soares, Simetrias Globais e Locais em Teorias de Calibre, Tese de Doutorado, IME-USP, Março de 2007. 